Florida International University FIU Digital Commons

$11-8-2016$

\title{
Magnetoelectric Nanoparticles: Paradigm Shift in Biomolecular Diagnostics
}

Chooda Mani Khanal

Florida International University, ckhan001@fiu.edu

DOI: $10.25148 /$ etd.FIDC001251

Follow this and additional works at: https://digitalcommons.fiu.edu/etd

Part of the Electrical and Computer Engineering Commons

\section{Recommended Citation}

Khanal, Chooda Mani, "Magnetoelectric Nanoparticles: Paradigm Shift in Biomolecular Diagnostics" (2016). FIU Electronic Theses and Dissertations. 2974.

https://digitalcommons.fiu.edu/etd/2974

This work is brought to you for free and open access by the University Graduate School at FIU Digital Commons. It has been accepted for inclusion in FIU Electronic Theses and Dissertations by an authorized administrator of FIU Digital Commons. For more information, please contact dcc@fiu.edu. 


\section{FLORIDA INTERNATIONAL UNIVERSITY}

Miami, Florida

MAGNETOELECTRIC NANOPARTICLES: PARADIGM SHIFT IN BIOMOLECULAR DIAGNOSTICS

A dissertation submitted in partial fulfillment of the

requirements for the degree of

DOCTOR OF PHILOSOPHY

in

ELECTRICAL ENGINEERING

by

Chooda M. Khanal 
To: Interim Dean Ranu Jung

College of Engineering and Computing

This dissertation, written by Chooda M. Khanal, and entitled Magnetoelectric Nanoparticles: Paradigm Shift in Biomolecular Diagnostics, having been approved in respect to style and intellectual content, is referred to you for judgement.

We have read this dissertation and recommend that it be approved.

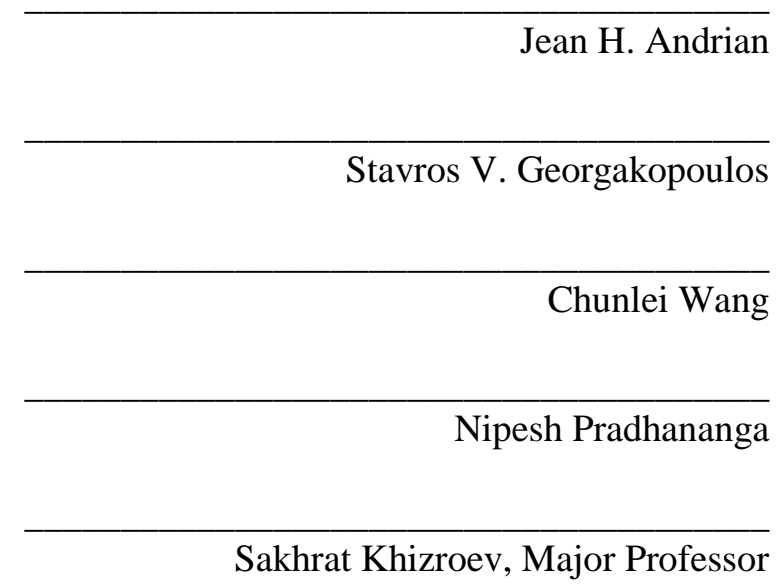

Date of Defense: November 08, 2016

The dissertation of Chooda M. Khanal is approved.

Interim Dean Ranu Jung
College of Engineering and Computing

College of Engineering and Computing

Andrés G. Gil

Vice President for Research and Economic Development and Dean of the University Graduate School

Florida International University, 2016 
(C) Copyright 2016 by Chooda M. Khanal All rights reserved. 


\section{DEDICATION}

I dedicate this dissertation to my beloved parents. 


\section{ACKNOWLEDGMENTS}

I would like to express great appreciation to my major advisor Professor Sakhrat Khizroev for the time and care for designing and refining my dissertation, and guiding me through my research. Without his mentorship this work would not have been possible. When I was down with an incurable disease called Pulmonary Arterial Hypertension, his encouragement not only gave me hope of survival but also lead me to continue my Ph.D. He is more than just an advisor. I would like to thank Prof. Nipesh Pradhananga, Prof. Chunlei Wang, Prof. Jean H. Andrian and Prof. Stavros V. Georgakopoulos for their helpful suggestions and comments.

I would like to thank my cardiologist Dr. Javier Jimenez from South Miami Hospital for his guidance on my health related research. I would also like to thank Prof. Bernard Gerstman and Prof. Prem P. Chapagain for their support during my stay at biophysics research group at the department of Physics at FIU. I would also like to thank my lab mates Alexandra Rodzinskaia, Tiffanie Stewart, Ping Wang, Abhignyan Nagesetti and Emmanuel Stimphil who have been very cooperative and helpful during experiments in the lab and beyond. I would like to thank my friends of my country of birth, Nepal for their love and support.

Finally, I would like to thank my dad, mom and family who have always encouraged me to fulfill my dreams. I would like to thank my wife Sarita, and my two sons Hrit Mani and Hriman Mani for the inspiration and in laws who has always supported while home. 


\section{ABSTRACT OF THE DISSERTATION \\ MAGNETOELECTRIC NANOPARTICLES: PARADIGM SHIFT IN \\ BIOMOLECULAR DIAGNOSTIC}

by

Chooda M. Khanal

Florida International University, 2016

Miami, Florida

Professor Sakhrat Khizroev, Major Professor

Recently discovered multiferroic nanoparticles (MFNs) known as magnetoelectric nanoparticles (MENs) promise to revolutionize next-generation biomedical diagnostic techniques and enable rapid and cost-effective screening of lethal diseases. Due to the quantum-mechanically caused intrinsic magnetoelectric (ME) effect, these nanoparticles display strongly coupled magnetic and electric moments and thus provide a unique pathway to monitor and optionally control intrinsic characteristics of bio organisms via application of external magnetic fields. Due to the ME effect, when placed in the microenvironment of a biomolecular sample, MENs' magnetic properties change to reflect the molecular nature of the sample. Such an unprecedented intrinsic connection to cells and microorganism's intrinsic characteristics can pave a way to a new diagnostic paradigm.

A novel concept of portable biomolecular screening device based on continuous wave nuclear magnetic resonance is being studied to identify microorganisms like bacteria, virus and cancer cell lines. For the first time, MENs have been used to modify NMR spectra and thus enable identification of different cancer cell lines from each other as well as from 
their normal counterparts at the sub-cellular level. This approach can be used for both in vitro and in vivo diagnostics. In addition, blood samples of Pulmonary Arterial Hypertension (PAH) patients with severe heart and lung conditions were used to study the activity of MENs with prothrombin, the clotting factor of the blood. MENs has significant interaction with prothrombin. MENS can be used for diagnostics of several diseases associated with blood and cancer and it may be used as a therapeutic agent. The experiment proved that the MENs are safe and nontoxic carriers of therapeutic drugs. 


\section{TABLE OF CONTENTS}

CHAPTER

PAGE

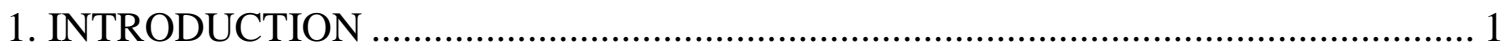

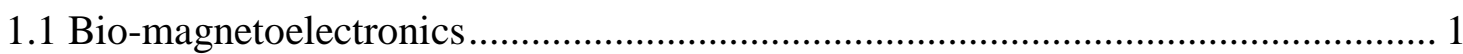

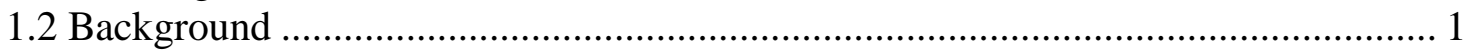

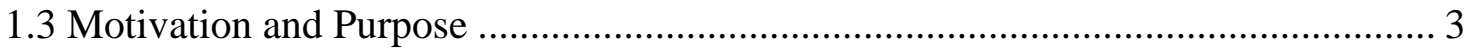

2. MAGNETOELECTRIC NANOPARTICLES....................................................... 6

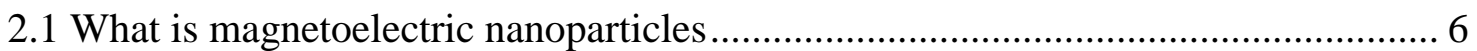

2.2 Novelty and significance of the proposed nanotechnology................................ 9

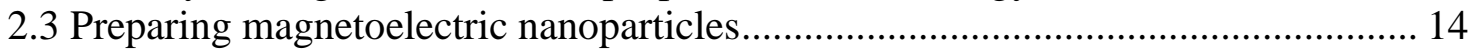

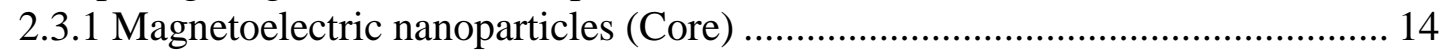

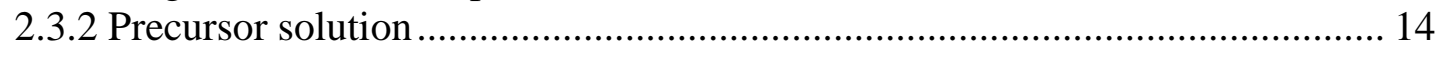

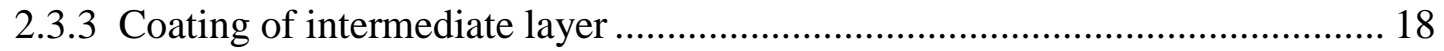

3. DEVICE PRINCIPLE AND DESIGN …......................................................... 21

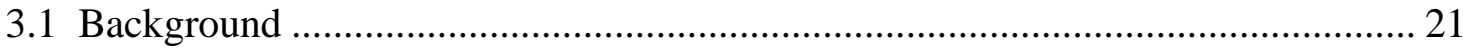

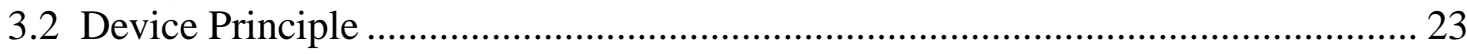

4. SIGNATURE IDENTIFICATION OF BACTERIAL SAMPLE ............................. 29

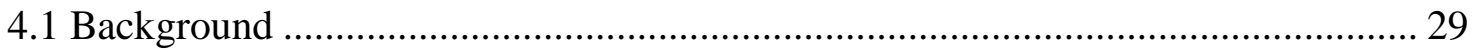

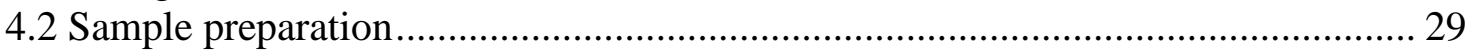

4.3 Device optimization and bacterial measurement ............................................ 36

5. SIGNATURE IDENTIFICATION OF CANCER CELL LINES ............................. 46

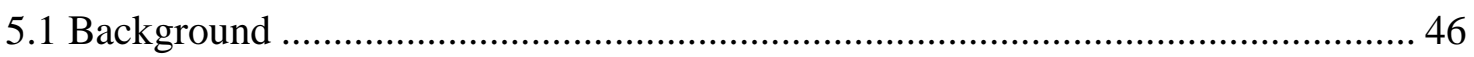

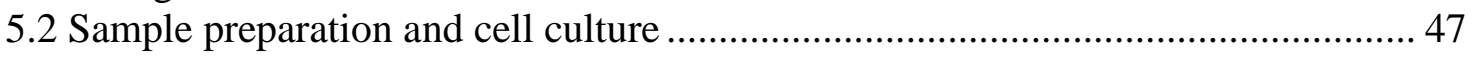

5.3 Measurement of Cancer Cells ....................................................................... 50

6. SIGNATURE ANALYSIS OF PROTHROMBIN ….......................................... 56

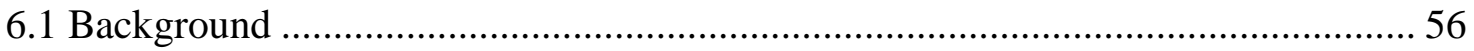

6.2 Prothrombin time and international normalized ratio ...................................... 56

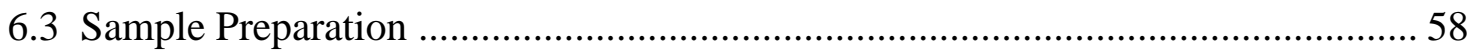

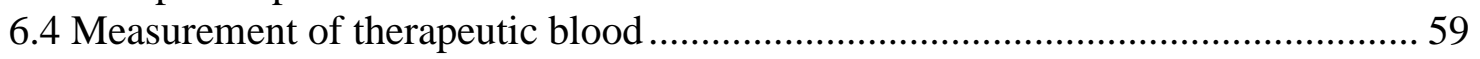

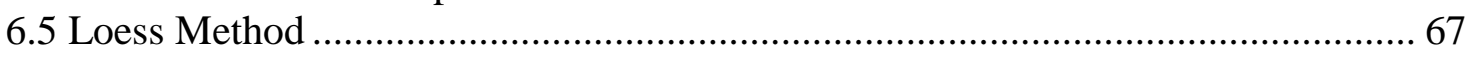

6.6 Sample blood with MENs coated with GMO and EDC................................... 69

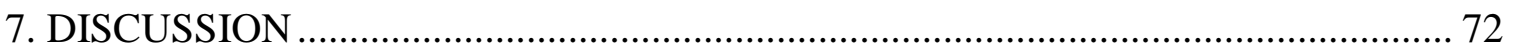




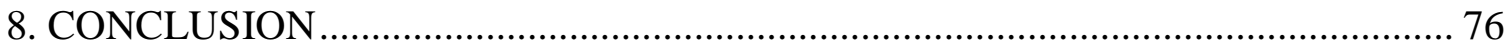

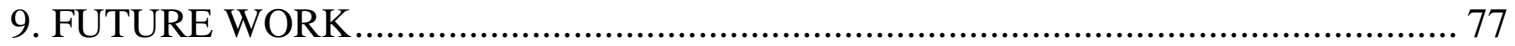

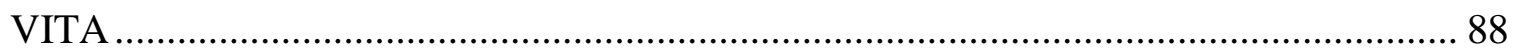




\section{LIST OF FIGURES}

FIGURE

Figure 1: The relationship between the magneto-electric and multiferroic materials. ........7

Figure 2. (a) M-H hysteresis loops for 30-nm MENs measured in a wide temperature range from 4 to $300 \mathrm{~K}$. The inset shows A TEM image showing a coreshell structure of 30-nm MENs. b) Blocking temperature measurement curves including zero-field cooling (ZFC), field cooling (FC) and field warming (FM) curves at a field of 100 Oe.

Figure 3: (a) and (b) show M-H hysteresis loops of 30-nm MENs and traditional 20$\mathrm{nm}$ magnetic nanoparticles (MNs) without any ME effect, respectively, measured via vibrating sample magnetometry (VSM).

Figure 4: Transmission Electron Microscopy (TEM) image showing the two-phase core-shell nature of $30 \mathrm{~nm}$ MENs.

Figure 5: Screen shot of AFM image of $10 \mathrm{~nm}$ nano-particles .16

Figure 6: Screen shot of AFM image of $100 \mathrm{~nm}$ nano-particles

Figure 7: Energy dispersion spectroscopy (EDS) results depict the composition of magnetoelectric nanoparticles.

Figure 8: Energy Dispersion Spectroscopy (EDS) results depict the composition of GMO coated magnetoelectric nanoparticles.

Figure 9: A basic device implementation for rapid screening of bacteria, viruses, and other cellular microorganisms.

Figure 10: Schematic illustration of a HIV virus with characteristic protruding proteins/ receptors. Nanoparticles bind to these proteins. .25

Figure 11: Spectra of normal cell line (red), cancer cell line f7 (black) and t47 (green) ..27

Figure 12: AFM image of Pseudomonas aeruginosa in a medium without MENs (top two) and with MENs (bottom two)

Figure 13: Figure 3.5: AFM images of Escherichia coli in a medium without MENs (top two) and with MENs (bottom two) 
Figure 14: AFM images of Serratia marcescens in a medium without MENs (top two) and with MENs (bottom two).

Figure 15: AFM images of Bacillus cereus in a medium without MENs (top two) and with

MENs (bottom two)

Figure 16: SEM images of pseudomonas aeruginosa without MENs (top two) and with MENs (bottom two) with different magnifications as shown in the label..34

Figure 17: SEM images of escherichia coli without MENs (top two) and with MENs (bottom two) with different magnifications as shown in the label.

Figure 18: SEM images of serratia marcescens without MENs (top two) and with MENs (bottom two) with different magnifications as shown in the label.

Figure 19: Figure 3.11: SEM images of bacillus cereus without MENs (top two) and with MENs (bottom two) with different magnifications as shown in the label..35

Figure 20: NMR Absorbance. A) Nanoparticles in the solution determine the strength of the absorbance. B) Bacterial solutions have a very weak absorbance..............36

Figure 21: Pelleted $1 \mathrm{ml} 1 \mathrm{X}$ bacteria, suspended in $20 \mu \mathrm{l}$ saline, added $20 \mu \mathrm{l}$ $50 \mathrm{mg} / \mathrm{ml} \mathrm{MENs.}$

Figure 22: Phase Optimization for MENs. A) MENs signal is strongest around a phase of 300. B) An example of a solution of bacteria with no MENs showing weak signal even at optimized settings.

Figure 23: MENs Provide Best Blind Differentiation. A) MENs being used to identify blinds. B) Traditional magnetic nanoparticles being used to identify blinds.

C) GMO-coated MENs being used to identify blinds.

Figure 24: Frequency Sweep. The field sweep data used is consistent with the frequency sweep data, but is not better for identifying bacterial blinds....

Figure 25: A) Four bacterial species were used to demonstrate the unique MENs interacting properties of each. B) Blind identities were successfully determined using the reference: $1=S$. marcescens, $2=B$. cereus, $3=P$. aeruginosa, 4=E. coli.

Figure 26: Eyeball comparison of MENs and Bacillus Cereus

Figure 27: Signature curve of Bacillus Cereus 
Figure 28: Eyeball comparison of MENs and Serratia marcescens .42

Figure 29: Signature curve of Serratia Marcescens ....................................................42

Figure 30: Eyeball comparison of MENs and Escherichia Coli ..................................43

Figure 31: Signature curve of Escherichia Coli ...................................................43

Figure 32: Eyeball comparison of MENs and Pseudomonas Aeruginosa .......................44

Figure 33: Signature curve of Pseudomonas Aeruginosa ...........................................44

Figure 34: Signature curves of Bacillus Cereus (blue), Serratia Marcescens (red), Escherichia

Coli (green), and Pseudomonas Aeruginosa (purple) ....................................45

Figure 35: AFM image pair of endothelial cells (z-height (left), phase (right)) ...............49

Figure 36: AFM image pair (z-height (left), phase (right)) of glioblastoma cells (a) without MENs, (b) with MENs...................................................................50

Figure 37: CW-NMR spectra of cells without MENs for ovarian carcinoma cells

SKOV-3, breast adenocarcinoma cells MCF-7 and glioblastoma cells U87-MG........51

Figure 38: CW-NMR Spectra of (a) U87-MG, (b) SKOV-3, (c) MCF-7, and (d) Brain

Endothelial Cells with traditional magnetic nanoparticles ..............................52

Figure $39 \mathrm{CW}-\mathrm{NMR}$ spectra of cells incubated with MENs for (a) glioblastoma cells U87-MG, (b) ovarian carcinoma cells SKOV-3, (c) breast adenocarcinoma cells MCF-7, and (d) normal brain endothelial cells

Figure 40: Comparison of $10 \mathrm{~nm}$ MENs background (blue) and MENs with blood sample (red)

Figure 41: Signature curve of $10 \mathrm{~nm}$ MENs with sample blood

Figure 42: Comparison of $30 \mathrm{~nm}$ MENs background (green), MENs with blood sample (blue) and MENs and blood sample with magnetization (red).

Figure 43: Signature curve of $30 \mathrm{~nm}$ MENs with sample blood (blue) and MENs and sample blood with magnetization (red)......

Figure 44: Comparison of $100 \mathrm{~nm}$ MENs background (blue), MENs with blood sample (red) and MENS and blood sample with magnetization (green) 
Figure 45: Signature curve of $100 \mathrm{~nm}$ MENs with sample blood (blue) and MENs and sample blood with magnetization (red)

Figure 46: CW NMR Spectra of MENs with therapeutic blood of measured INR 1.4 ....65

Figure 47: CW NMR Spectra of MENs with therapeutic blood of measured INR 3.1 ....66

Figure 48: CW-NMR Signature comparison of blood samples with measured INR 1.4 (blue) and 3.1 (red) ...............................................................................66

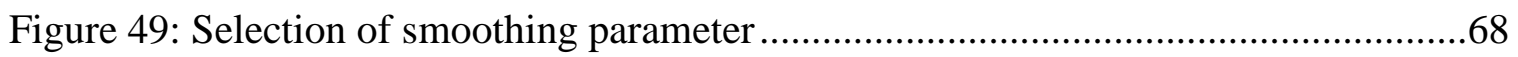

Figure 50: Figure 5.5: Curve fitting with smoothing parameter 0.053 ..........................68

Figure 51: Comparison of GMO and EDC coated $10 \mathrm{~nm}$ MENs with blood sample .......70

Figure 52: Comparison of GMO and EDC coated $100 \mathrm{~nm}$ MENs with blood sample .....70

Figure 53: Deformation of arteries with neo-hookean coefficient.................................78

Figure 54: Schematic diagram of drug loaded MENs with critical field entering

PASMC 


\section{LIST OF ACRONYMS}

\begin{tabular}{|c|c|}
\hline AFM & Atomic Force Microscopy \\
\hline ALL & Acute Lymphoblastic Leukemia \\
\hline COMPERA & $\begin{array}{l}\text { Comparative, Prospective Registry of Newly Initiated Therapies for } \\
\text { Pulmonary Hypertension }\end{array}$ \\
\hline $\mathrm{CBC}$ & Complete Blood Count \\
\hline CVD & Cardiovascular Disease \\
\hline DI & Deionized \\
\hline EDC & Ethyl Dimethylaminopropyl Carbodiimide \\
\hline EDS & Energy Dispersion Spectroscopy \\
\hline EDTA & Ethylene-diamine-tetraacetic Acid \\
\hline EPR & Enhanced Permeability and Retention \\
\hline ESR & Electron Spin Resonance \\
\hline $\mathrm{FC}$ & Functional Class \\
\hline FEM & Finite Element Method \\
\hline FTIR & Fourier Transform Infrared Spectroscopy \\
\hline GMO & Glycerol Monooleate \\
\hline HIV & Human Immunodeficiency Virus \\
\hline ICD & International Classification of Diseases \\
\hline INR & International Normalized Ratio \\
\hline ISI & International Sensitivity Index \\
\hline ME & Magneto-electric \\
\hline
\end{tabular}




\begin{tabular}{|c|c|}
\hline MEN & Magneto-electric Nanoparticles \\
\hline MFM & Magnetic Force Microscopy \\
\hline $\mathrm{MFN}$ & Multiferroic Nanoparticles \\
\hline MNB & Magnetic Nanoparticle Bio-sensing \\
\hline MNPT & Mean Normal Prothrombin Time \\
\hline MOKE & Magneto-optical Kerr Effect \\
\hline MPI & Magnetic Particle Imaging \\
\hline MRI & Magnetic Resonance Imaging \\
\hline NAMD & Nanoscale Molecular Dynamics \\
\hline $\mathrm{NIH}$ & National Institutes of Health \\
\hline NMR & Nuclear Magnetic Resonance \\
\hline NYHA & New York Heart Association \\
\hline PAH & Pulmonary Arterial Hypertension \\
\hline PASMC & Pulmonary Arterial Smooth Muscle Cells \\
\hline PBS & Phosphate Buffered Saline \\
\hline PCR & Polymerase Chain Reaction \\
\hline $\mathrm{PH}$ & Pulmonary Hypertension \\
\hline PPMS & Physical Property Measurement System \\
\hline PPT & Patient Prothrombin Time \\
\hline PT & Prothrombin Time \\
\hline PZT & Lead Zirconate Titanat \\
\hline $\mathrm{RBC}$ & Red Blood Cell \\
\hline
\end{tabular}




$\begin{array}{ll}\text { RV } & \text { Right Ventricle } \\ \text { SAS } & \text { Statistical Analysis Software } \\ \text { SCOV-3 } & \text { Human Ovarian Carcinoma Cell } \\ \text { TEM } & \text { Transmission Electron Microscopy } \\ \text { VEM } & \text { Viscoelastic Method } \\ \text { VMD } & \text { Visual Molecular Dynamics } \\ \text { VSD } & \text { Ventricular Septal Disease } \\ \text { VSM } & \text { Vibrating Sample Magnetometry } \\ \text { WBC } & \text { White Blood Cell } \\ \text { WHO } & \text { World Health Organization } \\ \text { ZFC } & \text { Zero Field Cooling }\end{array}$




\section{INTRODUCTION}

\subsection{Bio-magnetoelectronics}

A recently discovered class of multiferroic nanoparticles (MFNs) known as magnetoelectric nanoparticles (MENs) have potential to become useful tools for diagnosis, screening and targeted drug delivery in the near future [1-6]. These particles, which are not only electrically polarizable or magnetically polarizable but also exhibit both properties at the same time, are naturally designed to exploit the intrinsic characteristics of bio organisms. Due to the quantum-mechanical origin of their magnetoelectric (ME) coupling, these nanoparticles display strongly coupled magnetic and electric moments and thus provide a unique pathway to monitor and optionally control intrinsic characteristics of bio organisms via the application of external magnetic fields. The ME coupling of MENs surface-conjugated with a biomolecular sample strongly depends on the molecular nature of the sample and thus opens the door to a new diagnostic paradigm.

For the first time, this project is dedicated to exploiting the unique properties of multifunctional nanoparticles for rapid screening and unprecedented diagnostic of microorganisms and cells at the molecular level.

\subsection{Background}

Rapid and accurate identification of microorganisms, including bacteria and viruses, is critical for improving the state of care of infectious diseases. A lack of rapid identification and therapeutics, for example, allowed the 2014 Ebola outbreak to be one of the most widely spread and persistent epidemics in recent times [7]. Recently developed lab-on-a-chip technology that can detect the traces of Ebola virus has made it possible for 
rapid diagnostics and testing in the field, although it still requires testing in a special biosafety facility due to the highly infectious nature of the virus [8]. Despite the world's focus on the Zika virus, it has not decimated spreading across the nations, thanks to the slow and limited screening procedures [9-11]. Last but not least, the importance of the ability to rapidly screen cell cultures for different cancers and identification of cancer at the sub-cellular level is hard to overestimate especially today when cancer is number one cause of death. The traditional screening approach is to use bacterial culture and biochemical staining [12-18]. This technique, unfortunately, requires a tedious sample preparation protocol and is relatively slow (a matter of hours and sometimes days), and is limited to a few biomarkers. On a positive side, an advanced approach based on polymerase chain reaction (PCR) is exorbitant to be used in most clinics and small office environments $[19,20]$.

In addition, it is imperative to understand the functionality of proteins in our body that play a vital role for disease prevention and cure. There are many applications of nanotechnology in computer engineering, sensing, biology and medicine, pathogen detection etc.; however, not much has been done regarding the identification of diseases at the cellular or even molecular level [2-4,21-25]. Most of the modern nanomedicine development relates to drug delivery through blood vessels; the toxicity of nanoparticles has been discussed but very little has been done to understand the effect of nanoparticles on the blood coagulation system [26-29]. We attempt to give a new angle to nanoparticles from the perspective of diagnostics at the cellular level. We use MENs to distinguish various cancer cells from each other and from their normal counterparts, obtain the 
signature of prothrombin and its functionality using magnetoelectric nanoparticles, and consider other applications.

Our group has for the first time designed and investigated a device for sensitive and rapid screening for various biological entities such as bacteria, viruses, cancer cells, and other biomolecular structures. Over the past several years, we concocted this device on the principle of a small package and low-cost in order to make it readily available even in the poorest healthcare facilities. This device is based on continuous wave nuclear magnetic resonance (NMR) technology.

\subsection{Motivation and Purpose}

Infectious diseases caused by bacteria and viruses continue to exact a substantial toll on health and health care resources in the USA and globally. Despite success in some areas of disease treatment, a lack of reliable and rapid screening of such microorganisms continue to challenge prevention and control of disease persistence [30].

Recently there has been increased interest in magnetic nanoparticle bio-sensing (MNB) [31-33]. Due to the new dimensionality provided by the presence of externallycontrolled magnetic moments, MNB promises to enable high-specificity screening and fast diagnostic of pathogens. Indeed, ideally one could envision an apparatus in which magnetic nanoparticles are used to connect the intrinsic information with regards to single cells, e.g. the electric charge profile on the surface of a cell emerged in a specific biological microenvironment (media), to external devices which can adequately process the magnetic information, e.g. nuclear magnetic resonance (NMR), magnetic resonance imaging (MRI), magnetic particle imaging (MPI), vibrating sample magnetometry (VSM), B-H looper for 
susceptibility measurements, magneto-optical Kerr microscopy, magnetic force microscopy (MFM), and others [34-36].

The other motivation of this dissertation is to maximize the drug delivery capabilities of the same nanoparticles used for screening. Studying the above diagnostic application involves deep understanding of mechanisms of the molecular interaction between nanoparticles and the microorganisms at the sub-cellular level and development of ways to control these intrinsic mechanisms. The knowledge obtained through this study is critical also for improving the more popular drug delivery applications of nanoparticles. For example, this knowledge will help achieve full payload carrying capacity and delivery of the fully loaded cargo in a manner where release close to $100 \%$ of the therapeutic drug is released on site and on-demand [2-4,37,38]. In modern drug delivery approaches, therapeutic drugs are attached to nanoparticles via covalent, non-covalent (electrostatic, Van der Waal, hydrogen), or ionic bonds. Modifying the surface properties (i.e. by controlling the surface charge and polarity) of the nanoparticles and their functionalization plays a key role in improving the attachment of the drug. Such goals are also being pursued in this particular study.

Most of the current nanoparticle based drug delivery systems are relatively inefficient due to their strong dependence on the intrinsic properties of the cell's physiological conditions such as temperature, $\mathrm{pH}$, enzymatic reactions, and others to deliver the cargo drug. As a result, the drug releasing process is extremely inefficient due to the dependence on the minute changes in the physiological conditions in the microenvironment of the cell. 
Using MENs instead of the traditional nanoparticles can overcome this roadblock because MENs provide an additional dimension to control delivery and release of the drug. 


\section{MAGNETOELECTRIC NANOPARTICLES}

\subsection{What is magnetoelectric nanoparticles}

Multiferroic nanoparticles (MFNs), which sometimes include the category of particles called magneto-electric nanoparticles (MENs), simultaneously show the properties of ferromagnetism and ferroelectricity. These MEN particles are magnetically as well as electrically polarizable and show all the properties- ferromagnetic, ferroelectric, multiferroic, and magnetoelectric. MFMs exhibits a strong quantum coupling of magnetic and electric properties and come under the multiferroic category, as they demonstrate both ferroelectric and ferromagnetic properties simultaneously $[1,5,6]$.

Figure 1 shows the relationship between the magneto-electric and multiferroic materials. Borocite $\left(\mathrm{Ni}_{3} \mathrm{~B}_{7} \mathrm{O}_{13} \mathrm{I}\right)$ was the first material reported to exhibit magneto-electric switching below $60 \mathrm{~K}$ [39]. Magnetoelectric effect in composite materials is the product tensor property given by the product of magnetostrictive effect (magnetic/ mechanical effect) in the magnetic phase and the piezoelectric effect (mechanical/ electrical effect) in the ferroelectric phase [40]. Magnetic to electric energy conversion exploits the advantages of properties from two different phases of the material, i.e. from the ferromagnetic phase, piezomagnetic or magnetostrictive property and from the ferroelectric phase, piezoelectric or electrostrictive property. Thus, when an external magnetic field $(\Delta H)$ is applied, magnetostrictive stress formed in the magnetic phase is transferred to the ferroelectric phase changing the polarization $(\Delta \mathrm{P})$ and the associated electric field $(\Delta \mathrm{E})$ due to the piezoelectric effect. Performance of magnetoelectric materials is determined by this transfer efficiency, which is referred to as the magneto-electric coefficient $(\alpha=\Delta \mathrm{P} / \Delta \mathrm{H})$ or as the magneto-electric voltage coefficient $\left(\alpha_{E}=\Delta E / \Delta H\right)$. Magneto-electric coefficients 
of various multiferroic materials are shown in Table 1. Electric flux density (D) in multiferroic materials as shown in the following equation is determined by the magnetoelectric coefficient tensor $\left(\alpha_{\mathrm{ij}}\right)$, permittivity tensor $\left(\varepsilon_{\mathrm{ij}}\right)$, and the piezoelectric coefficients $\left(e_{\mathrm{ij}}\right)$ in an applied vector magnetic, electric field, and external stress $\mathrm{S}[1,41]$.

$$
D_{i}=\sum_{j-1}^{j-3} \varepsilon_{i j} E_{j}+\sum_{j-1}^{j-6} e_{i j} S_{j}+\sum_{j-1}^{j-3} \alpha_{i j} H_{j}
$$
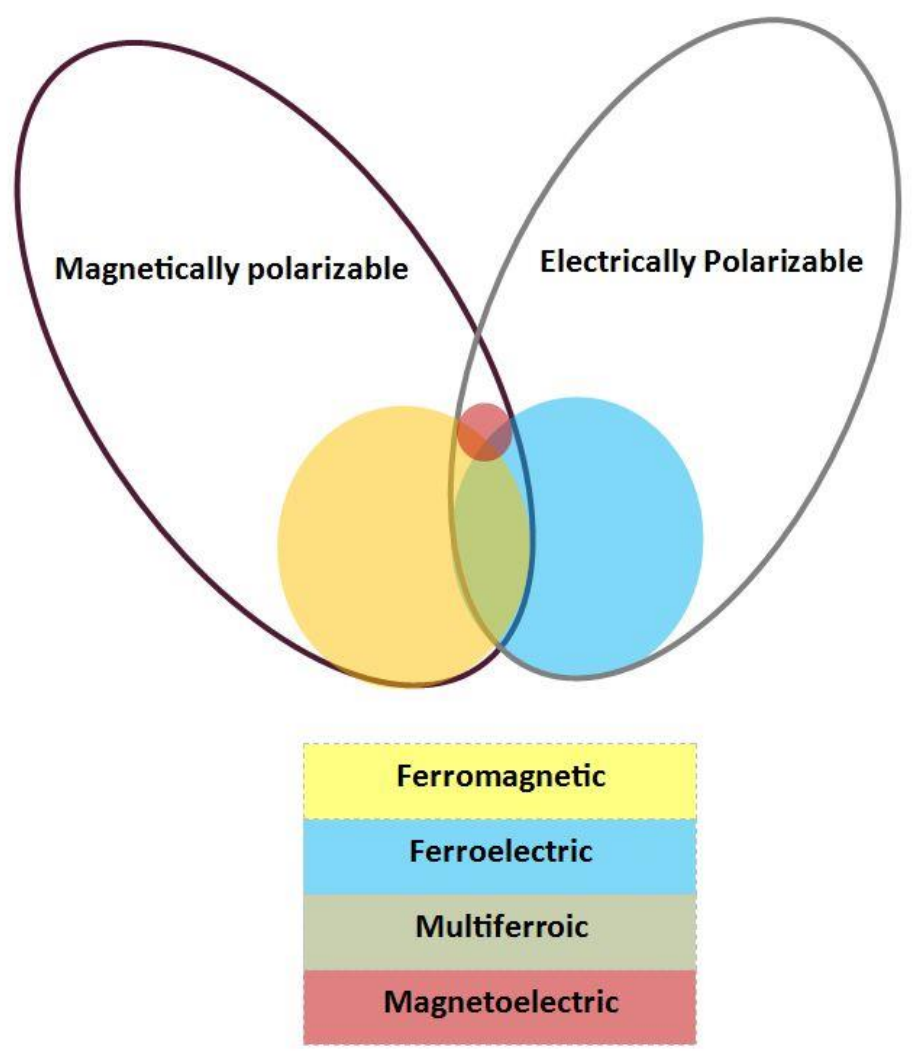

Figure 1: The relationship between the magneto-electric and multiferroic materials. 


\begin{tabular}{|c|c|c|c|}
\hline Morphology & Materials & $\begin{array}{c}\text { ME } \\
\text { coefficient }\end{array}$ & Ref \\
\hline Composite & $\mathrm{BaTiO}_{3}$ and $\mathrm{CoFe}_{2} \mathrm{O}_{4}$ & 50 & {$[42]$} \\
\hline Composite & Terfenol-D and PZT in polymer matrix & 42 & {$[43]$} \\
\hline $\begin{array}{c}\text { Laminated } \\
\text { composites }\end{array}$ & Terfenol-D in polymer matrix/PZT in polymer & 3,000 & {$[44]$} \\
\hline Laminate & matrix & 4,800 & {$[45]$} \\
\hline Laminate & $\mathrm{Terfenol-D/PZT}_{0.7} \mathrm{Sr}_{0.3} \mathrm{MnO}_{3} / \mathrm{PZT}$ & 60 & {$[46]$} \\
\hline Laminate & $\mathrm{NiFe}_{2} \mathrm{O}_{4} / \mathrm{PZT}$ & 1,400 & {$[46]$} \\
\hline
\end{tabular}

Table 1: Magneto-electric coefficients in two-phase systems.

Magnetoelectric materials can be used in two forms, i.e. either in the laminate form or in the particulate form. Using magnetoelectric materials in the particulate form is often the better option because of the excellent magneto-electric properties caused by the following reasons:

a. Shape effects: spherical shape of the particles provides better transfer of magnetostrictive stress from magnetic phase to the ferroelectric phase.

b. Zero-substrate clamping effect: laminates/films needs to be clamped, which restricts the magnetostrictive strains.

c. Binding efficiency: mechanical contact in the interface of particulate composites is much higher as compared to the laminate/films composites. (Note: laminate/films composites need to be either glued or sintered which often results in poor binding efficacies).

So far, magnetoelectric materials are extensively used in microwave filters, signal amplification devices, data storage devices, transducers, optical-diodes, power generation systems, high frequency switches, miniature-antennas, and spintronics [47-54], but in this 
dissertation, we present the biomedical applications of the magnetoelectric materials especially the application of detection of microorganisms.

MENs, also called "smart" nanoparticles, can controllably regenerate important physical functions on the surface of the cellular microorganism being tested. The MEN's underlying physics is a very intricate quantum-mechanical coupling between magnetoelectric spinel and ferromagnetic perovskite structures. This coupling allows the use of external magnetic fields to control local electric fields for sub-cellular diagnostic and targeted drug delivery into specific cells $[55,56]$.

\subsection{Novelty and significance of the proposed nanotechnology}

Due to the new dimensionality provided by the presence of externally-controlled magnetic moments, in magnetic nanoparticles, we have the capability to enable highspecificity screening and fast diagnostic of pathogens. Indeed, ideally one could envision an apparatus in which magnetic nanoparticles are used to connect the intrinsic information with regards to single cells, e.g. the electric charge profile on the surface of a cell emerged in a specific biological microenvironment (media), to external devices which can adequately process the magnetic information, however, the current progress in the area of novel technologies is still relatively slow. The challenge is to "connect" magnetic nanoparticles to the intrinsic information at the cellular or intra-cellular level. The fundamental nature of this intrinsic information is reflected in its electric-field characteristic; all of the previously mentioned microorganisms and cell structures have signature surface proteins/receptors which are inclined to be electrically polarized in a certain biological microenvironment and which are also the main binding sites for 
nanoparticles. The charge on different cell surfaces does not only differ by its amount but also by its distribution. Unfortunately, it is difficult to measure this information directly using electric fields alone because electric fields experience significant interference when they go through the media and as a result direct physical contacts would be required, which then makes the measurements both difficult and costly. In addition, the traditional approach of using nanoparticles for bio-sensing and as a drug delivery platform lacks the sufficient targeted delivery in a controlled environment application. Therefore, to overcome all the above challenges associated with the traditional magnetic nanoparticles, we have chosen to exploit MENs.

MENs can be used to enable externally controlled passive targeted delivery and release of therapeutic loads, e.g. specific peptides for regulating cell proliferation with high selectivity. Unlike modern active-targeting nanotechnology approaches, e.g. antibody- or ligand mediated therapies that interact with the immune system, MENs do not rely on the immune system to target the diseased cells. Instead, MENs use a physical force that is driven by the Coulomb interaction, which is self-triggered when the nanoparticles circulate past the targeted cells with a specific electric-field signature. This self-triggering mechanism can be either "pre-programmed" via defining the MENs saturation magnetization and the ME coefficient through a fabrication process or via application of an external magnetic field. Unlike conventional passive targeting, like enhanced permeability and retention (EPR), MENs do not need relatively large cell aggregates for targeting [57,58]. MENs-driven passive targeting is achieved at the cellular level through detection of specific stray electric field profiles. 
The drug-loaded MENs cross the membrane through field induced "nanoelectroporation" and release their load on demand. We proved our novel technique to show that the anti-cancer drug loaded in our smart particles penetrated through the membrane and completely eradicated tumor cells within 24 hours without affecting the normal cells $[2,4]$. We have also demonstrated the externally controlled on-demand release of anti-HIV drug using our smart nanoparticles as carriers [3].

Moreover, the fact that each cell possesses a unique intrinsic electric characteristic, MENs may also be used as magneto-electronic biomarker. Some cancer cannot be treated completely and also passes from generation to generation. The most common cause of treatment failure in acute lymphoblastic leukemia (ALL) remains relapse, occurring in approximately 15 to 20 percentage of patients [59-61]. In other words, a child with ALL may seem to be cured completely with chemotherapy treatment however about 15 to 20 percentage of the time it appears again during adulthood. The cancerous traces hidden in brain or sometimes in testicles (testicular relapse in male) cannot be treated by the conventional chemotherapy and remains unknown for years. There is no other way of finding out if the cancerous traces are still there in the body after treatment. So, exploiting the electric property of such cells with the MENs as a magneto-electronic biomarker may lead to the path of diagnosis and targeted therapy of such diseases.

Before we proceed, once again we would like to stress the importance of not confusing MENs with traditional magnetic nanoparticles (MNs) and the novelty and significance of proposed nanotechnology based on MENs. One of the importance of MENs over the traditional magnetic nanoparticles (MNs) is shown in Figure 3. MENs were 30- 
nm coreshell $\mathrm{CoFe}_{2} \mathrm{O}_{4} @ \mathrm{BaTiO}_{3}$ nanostructures consisting of the ferrimagnetic spinel core $\mathrm{CoFe}_{2} \mathrm{O}_{4}$ and the piezoelectric perovskite shell $\mathrm{BaTiO}_{3}$. MNPs were 20-nm $\mathrm{CoFe}_{2} \mathrm{O}_{4}$ ferrimagnetic nanostructures.

Following is the $\mathrm{M}-\mathrm{H}$ characteristics and blocking temperature measurement plot using vibrating sample magnetometer physical property measurement system (PPMS). A room-temperature Lakeshore vibrating sample magnetometer (VSM) with a 3-T magnetic field sweep was used to measure key magnetic properties of nanoparticles under study including the magnetization saturation and the magnetic coercivity. A cryogenic VSM Quantum Design PPMS with a 9-T superconducting magnet was used to measured M-H temperatures in a wide temperature range, from $4 \mathrm{~K}$ to over $300 \mathrm{~K}$.
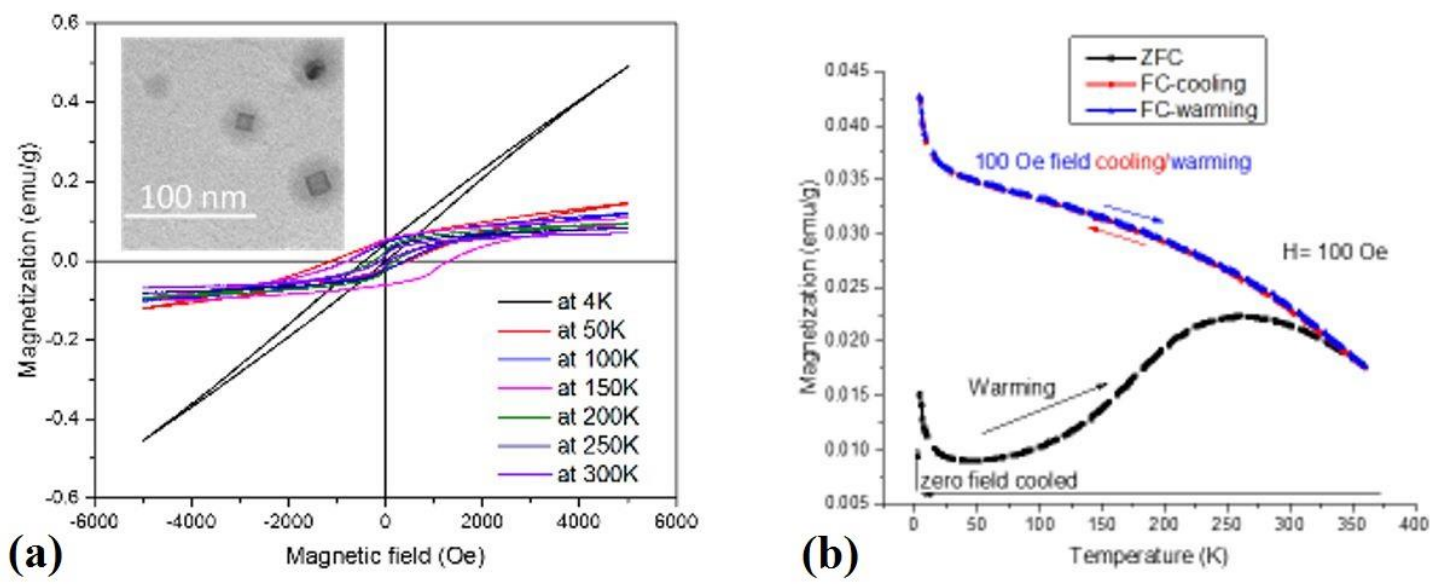

Figure 2. (a) M-H hysteresis loops for 30-nm MENs measured in a wide temperature range from 4 to $300 \mathrm{~K}$. The inset shows A TEM image showing a coreshell structure of 30-nm MENs. b) Blocking temperature measurement curves including zero-field cooling (ZFC), field cooling (FC) and field warming (FM) curves at a field of $100 \mathrm{Oe}$. 

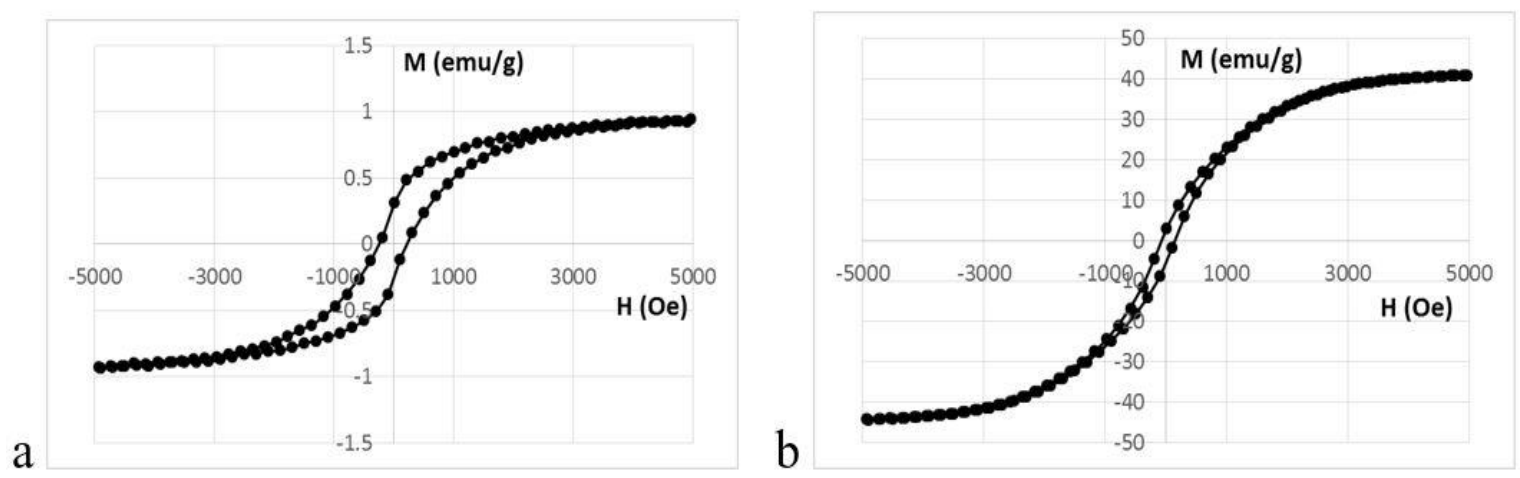

Figure 3: (a) and (b) show M-H hysteresis loops of 30-nm MENs and traditional 20-nm magnetic nanoparticles (MNs) without any ME effect, respectively, measured via vibrating sample magnetometry (VSM).

To understand the MENs' temperature dependence, typical M-H hysteresis loops of MENs in a temperature range from 4 to $300 \mathrm{~K}$ obtained via a cryogenic vibrating sample magnetometer Quantum Design PPMS are shown in Figure 2(a). The standard magnetization versus temperature curves under zero field cooling (ZFC) and non-zero field cooling (FC) conditions to determine the blocking temperature are shown in Figure 2(b). The blocking temperature is the temperature above which the nanoparticles become superparamagnetic. In this case, it is above $300 \mathrm{~K}$, which confirms that the nanoparticles are not superparamagnetic at room temperature despite their small size. The ME coefficient, $\alpha$, for these nanostructures is known to be in the range from 10 to over $100 \mathrm{mV}$ $\mathrm{cm}^{-1} \mathrm{Oe}^{-1}$

From the loops in Figure 3, one can see that MENs and MNs have saturation magnetizations of approximately 1 and $40 \mathrm{emu} / \mathrm{g}$, respectively, and coercivity fields of approximately 310 and 90 Oe, respectively. 


\subsection{Preparing magnetoelectric nanoparticles}

\subsubsection{Magnetoelectric nanoparticles (Core)}

$\mathrm{CoFe}_{2} \mathrm{O}_{4}$ particles (core) were prepared by the standard hydrothermal method $[2,62] .0 .58$ grams of Cobalt Nitrate (Hexahydrate) reagent $\left[\mathrm{Co}\left(\mathrm{NO}_{3}\right)_{2} \cdot 6 \mathrm{H}_{2} \mathrm{O}\right]$ and 1.6 grams of ferric nitrate $\left[\mathrm{Fe}\left(\mathrm{NO}_{3}\right)_{3} .9 \mathrm{H}_{2} \mathrm{O}\right]$ were dissolved in 150 milliliters of distilled water taken in a beaker. In a second beaker 2 grams of polyvinylpyrrolidone and 9 grams of sodium borohydride were dissolved in $50 \mathrm{ml}$ of distilled water. The two aqueous solutions were mixed by gently adding drop by drop from the second beaker to the first one using pipette and continuously stirring at $320 \mathrm{rpm}$ and then $1000 \mathrm{rpm}$. During this process, the beaker was placed in a heater at $70^{\circ} \mathrm{C}$. After the solution is completely incorporated, the stirrer was set to $300 \mathrm{rpm}$ and the temperature was set to $90^{\circ} \mathrm{C}$ for overnight (12 hours). Similar procedures for the preparation of core are implemented in the Personalized Nanomedicine lab at FIU for previously published journals [2-4].

\subsubsection{Precursor solution}

To prepare the precursor solution of $\mathrm{BaTiO}_{3}$, two beakers were taken. $150 \mathrm{~mL}$ of ethanol was mixed with 5 grams of citric acid and $240 \mathrm{uL}$ of titanium (IV) ispropoxide in the first beaker. 1 gram of citric acid and 0.145 gram of barium carbonate was dissolved in the second beaker containing $150 \mathrm{~mL}$ of deionized (DI) water. The two solutions were mixed and sonicated for 60 minutes.

Coreshell $\mathrm{CoFe}_{2} \mathrm{O}_{4}-\mathrm{BaTiO}_{3}$ multiferroic nanoparticles were prepared by mixing 0.5 $\mathrm{g}$ of $\mathrm{CoFe}_{2} \mathrm{O}_{4}$ nanoparticles core in the $\mathrm{BaTiO}_{3}$ precursor solution and the mixture was sonicated for 2 hours. Once the $\mathrm{CoFe}_{2} \mathrm{O}_{4}$ nanoparticles were thoroughly dispersed, the 
mixture was dried on the hot plate at $70^{\circ} \mathrm{C}$ overnight while continuously stirring. The obtained polymer precursor was subjected to $500^{\circ} \mathrm{C}$ (for $10 \mathrm{~nm}$ ), $600^{\circ} \mathrm{C}$ (for $30 \mathrm{~nm}$ ), $700^{\circ} \mathrm{C}$ (for 100nm) for 5 hours in a furnace (CMF-1100).

The prepared samples were imazed for the size verification. We used scanning probe miscroscopy, Transmission Electron Microscopy (TEM) [63] and Atomic Force Microscopy (AFM) [64]. All the samples were verified before use. TEM is an imaging technique where a beam of electrons is transmitted through a sample interacting with it as it passes through it. The image is formed by the interaction of the beams with the sample, details explained elsewhere [63]. Figure 4 is an image of a $30 \mathrm{~nm}$ sample taken by TEM. It clearly shows the two-phase core shell nature of MFMs. The scale bar is $100 \mathrm{~nm}$. Similarly, we took TEM images of $10 \mathrm{~nm}$ and $100 \mathrm{~nm}$ nano particles as well (not shown here).

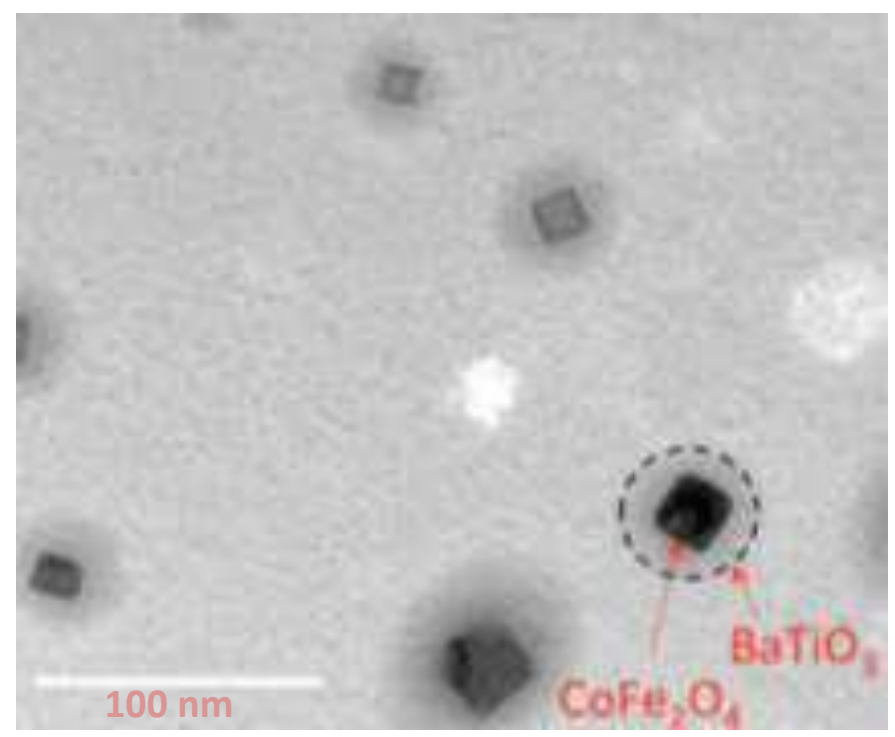

Figure 4: Transmission Electron Microscopy (TEM) image showing the two-phase coreshell nature of $30 \mathrm{~nm}$ MENs. 
The Atomic Force Microscope cannot show the two phase shell due to its different nature of imaging. The image is taken by feeling or touching the surface of the sample my its mechanical probe, and not by transmitting through the sample as in TEM. AFM takes an image by the reaction of the probe to the forces that the sample imposes on it as the tip of AFM runs across the surface of the sample that can be used to form an image of the three-dimensional shape (topography) of a sample surface at a high resolution. It can show the size of the particles in nanometer scale. Figure 5 and Figure 6 are the screen shot images of $10 \mathrm{~nm}$ and $100 \mathrm{~nm}$ respectively taken my AFM.

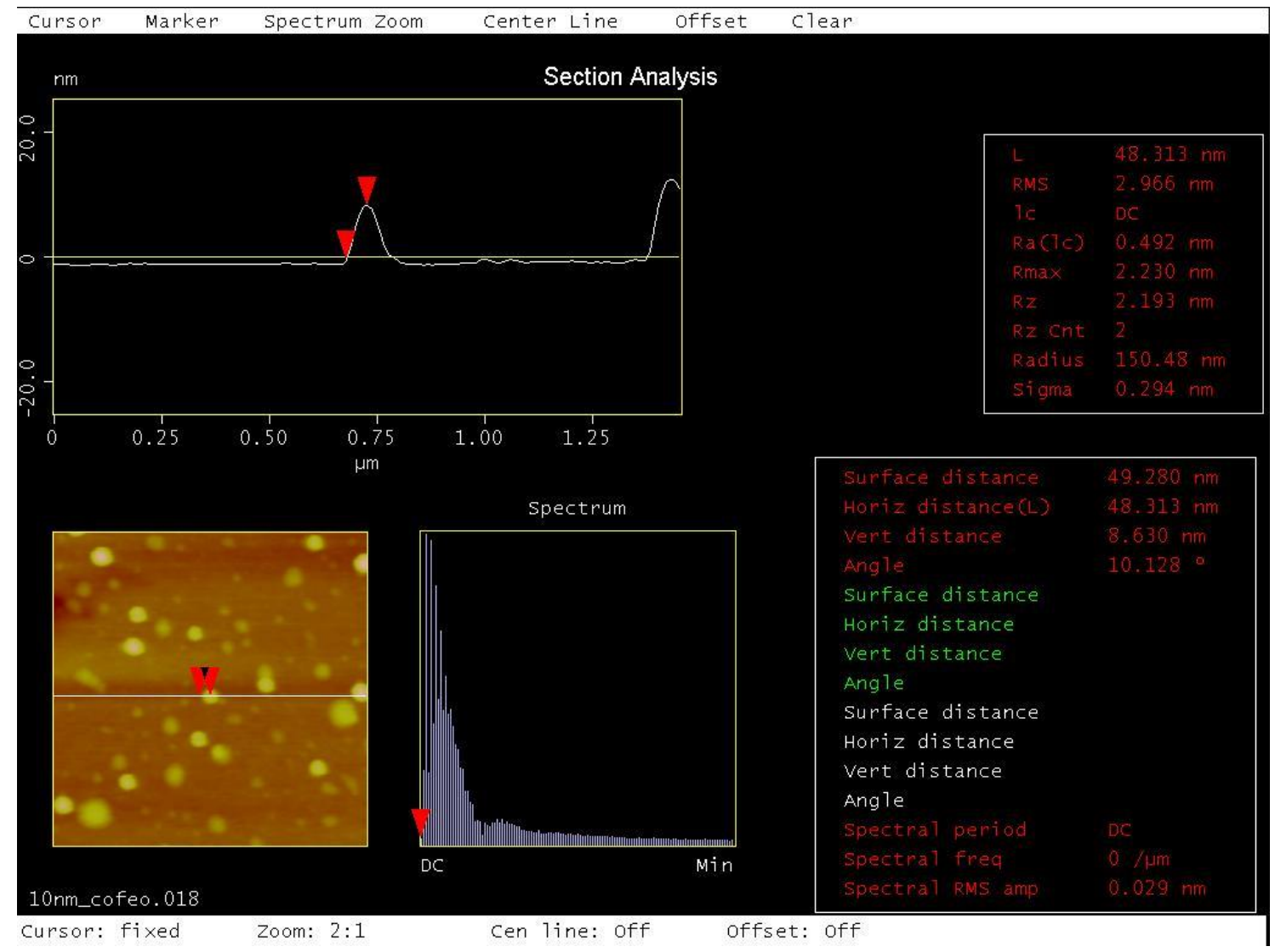

Figure 5: Screen shot of AFM image of $10 \mathrm{~nm}$ nano-particles. 


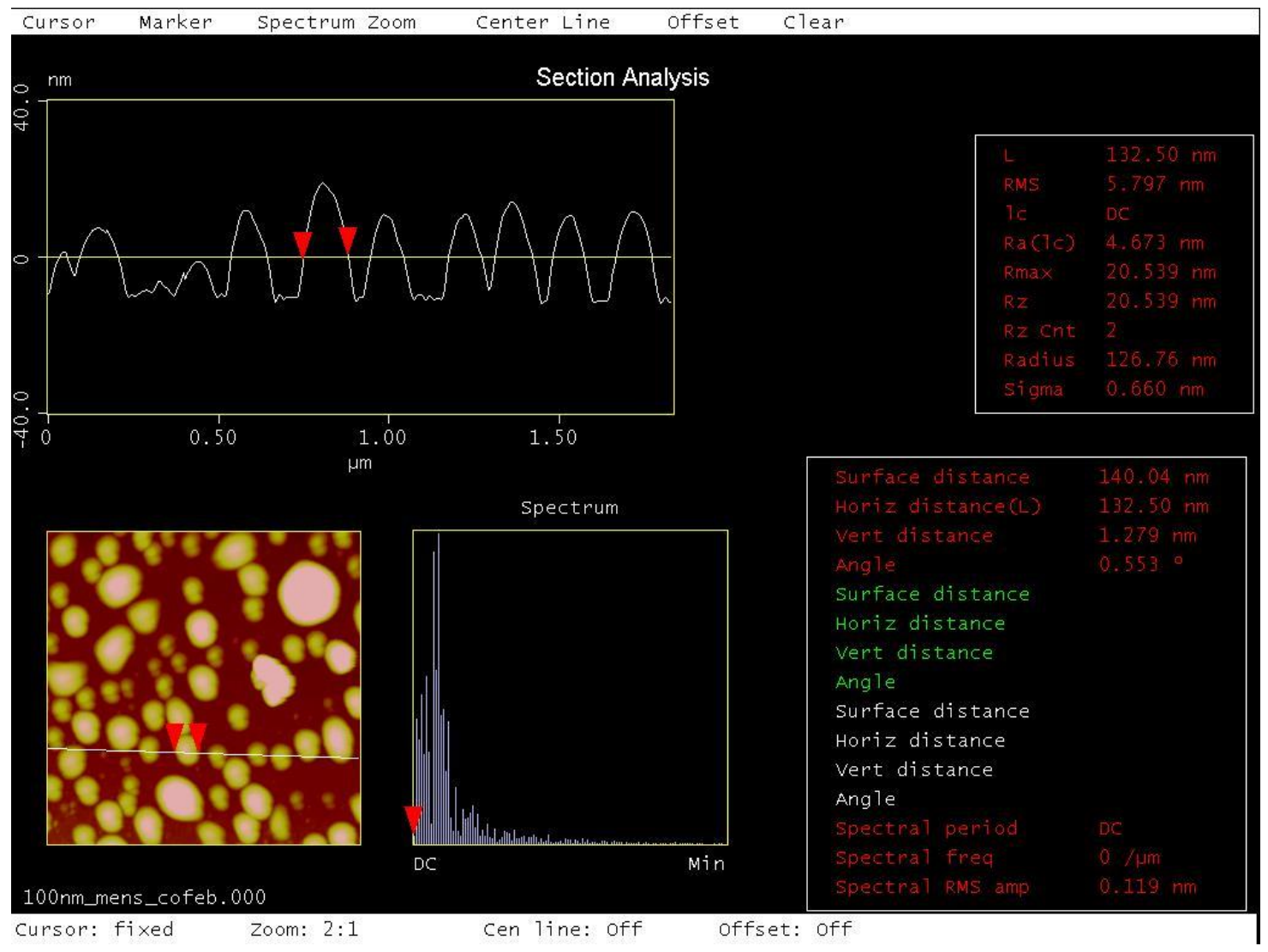

Figure 6: Screen shot of AFM image of $100 \mathrm{~nm}$ nano-particles.

The radius of curvature of AFM tip is on the order of $10 \mathrm{~nm}$, so there is always an additional margin of about $20 \mathrm{~nm}$ on the horizontal distance of the sample. For example, Figure 4 shows an image of 100-nm nanoparticles. The curser is placed at the two extremities of the particle and the horizontal distance shows about $130 \mathrm{~nm}$. The average size of the particles is $100 \mathrm{~nm}$. Similarly, Figure 3 is an image of $10 \mathrm{~nm}$ particles. The curser is placed at one edge and the center of the particle to measure the vertical distance of the particle rather than the horizontal distance. If the particle size is on the order of the radius of curvature of the tips, it is better to take the vertical distance to measure the particle size. Due to the difference in vertical and horizontal positioning approaches, the vertical 
distance and each of the two horizontal distances are measured with precisions of approximately 1 and $10 \mathrm{~nm}$, respectively.

\subsubsection{Coating of intermediate layer}

Due to the ultra-fine size of MENs and the low toxicity, they are promising candidates for medical applications. However, since the medicated particles are transported via blood, their sizes, surface charge and ME chemistry strongly affects the bioavailability of the particles inside the body. Not to mention that under some cases, if not properly treated and/or functionalized, the nanoparticles may become toxic and may not properly function for the desired external magnetic field for targeted delivery. Specific experiments require controllable dissociation of drug molecules from the nanoparticle surface by selecting appropriate intermediate layers, such as Glycerol monooleate (GMO), Tween-20, or Ethyl-3-(3-dimethylaminopropyl) carbodiimide (EDC). In our experiment we used a $2 \mathrm{~nm}$ of the intermediate layers of GMO and EDC.

In both cases (to coat with GMO and EDC, respectively), as the first step $20 \mathrm{mg}$ of MENs were added to phosphate-buffered saline (PBS) buffer solution. Then, $40 \mathrm{uL}$ of GMO solution $(1 \mathrm{mg} / \mathrm{ml})$ was added to the solution and incubated for an hour while slowly rotating to achieve uniform coatings. After completing the incubation process, the particles were washed by centrifuging to 33,000 revolutions per minute (rpm) for 5 minutes. After spinning, any GMO bubbles and PBS were removed gently. PBS was added again to the sediments, vortexed, and centrifuged again to 40,000 rpm for 5 minutes. The washing process was repeated 3 to 4 times until all unbounded GMO was completely removed from the solution. 
We used Energy Dispersion Spectroscopy (EDS), an analytical technique used for the elemental analysis or chemical characterization of a sample. Phillips CM-200 $200 \mathrm{kV}$ Transmission Electron Microscope (TEM) with Energy Dispersive Spectroscopy (EDS) option was used to obtain TEM images and EDS profiles. Figure 7 shows the composition of MENs and Figure 8 depicts the composition of GMO coated nanoparticles.

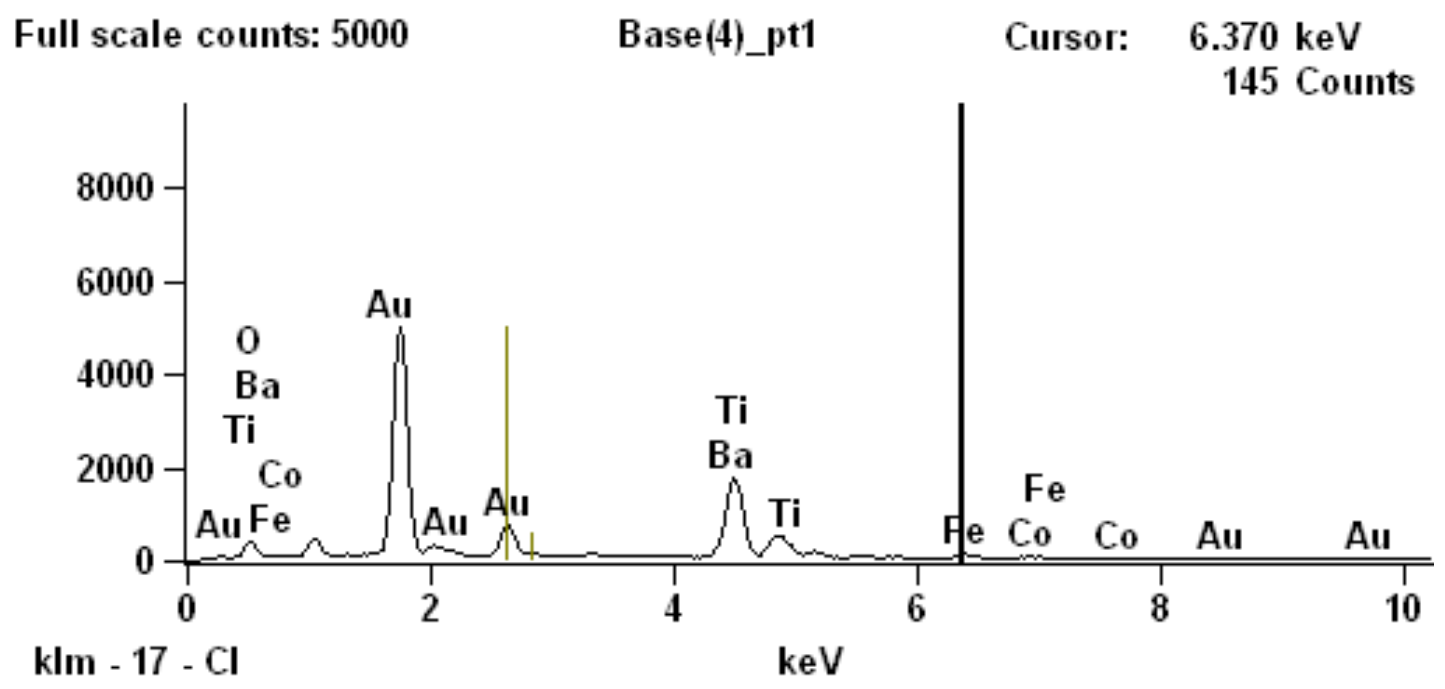

Figure 7: Energy dispersion spectroscopy (EDS) results depict the composition of magnetoelectric nanoparticles. 
Full scale counts: $\mathbf{1 8 0 6}$

$$
\text { Base(3)_pt1 }
$$

Cursor: $\quad 0.793 \mathrm{keV}$

89 Counts

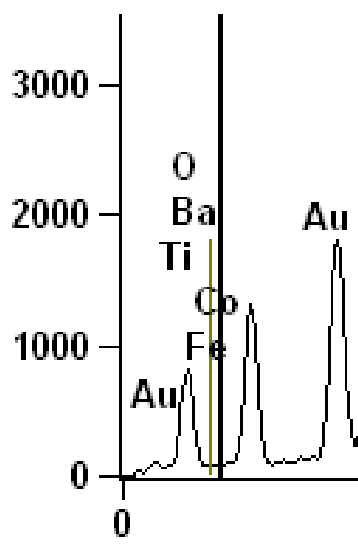

klm - 26 - Fe Lb3

keV

Figure 8: Energy Dispersion Spectroscopy (EDS) results depict the composition of GMO coated magnetoelectric nanoparticles. 


\section{DEVICE PRINCIPLE AND DESIGN}

\subsection{Background}

We designed a nanodevice for rapid screening and signature identification of cells and other microorganisms. We investigated the invention on cancer cells, different bacteria, viruses, prothrombin and other biomolecular structures over the course of several years. The principle of this cost effective and small package format device is based on continuous wave nuclear magnetic resonance (NMR) technology. NMR is a physical phenomenon in which nuclei in a magnetic field absorb and re-emit electromagnetic radiation. The energy is at a specific resonance frequency which depends on the strength of the magnetic field and the magnetic properties of the atoms. Protons and neutrons inside the nucleus of an element possess a magnetic dipole moment $(\mu)$ which is similarly characterized by an intrinsic spin quantum number like an electron spin. If a nucleus has an even number of nuclei for example ${ }^{12} \mathrm{C}_{6}$, which has 6 protons and 6 neutrons, these spins are paired against each other summing to zero overall spin or magnetic dipole moment. However, in nuclei like ${ }^{1} \mathrm{H}_{1}$ and ${ }^{19} \mathrm{~F}_{9}$, the nucleus does not possess a net zero spin, so these nuclei have a finite magnetic dipole moment.

It is commonly recognized that sensitive and rapid identification of blood cells and microorganisms including bacteria and viruses is critical for improving the state of care of infectious diseases. The traditional approach is to use bacterial culture and biochemical staining. Unfortunately, this technique requires a tedious sample preparation procedure and is thus relatively slow (a matter of hours and sometimes days), and, not to mention, is limited to a few notable biomarkers. A more advanced approach based on polymerase chain 
reaction (PCR) remains cost-ineffective for being used in most clinics and small office environments.

Therefore, there has recently been increased interest in magnetic nanoparticle biosensing (MNB). Due to the new dimensionality provided by the presence of externallycontrolled magnetic moments, MNB promises to enable high-specificity screening and fast diagnostic of pathogens. Indeed, ideally one could envision an apparatus in which magnetic nanoparticles are used to connect the intrinsic information with regards to single cells, e.g. the electric charge profile on the surface of a cell located in a specific biological microenvironment (media), to external devices which can then adequately process the magnetic information, e.g. nuclear magnetic resonance (NMR), magnetic resonance imaging (MRI), magnetic particle imaging (MPI), vibrating sample magnetometry (VSM), B-H looper for susceptibility measurements, magneto-optical Kerr microscopy, magnetic force microscopy (MFM), and others [31-36].

However, the current progress in the area of novel technologies is still relatively slow. The challenge is to "connect" magnetic nanoparticles to the intrinsic information at the cellular or intra-cellular level. The fundamental nature of this intrinsic information is reflected in its electric-field characteristic; all the previously mentioned microorganisms and cell structures have signature surface marker proteins and receptors which are inclined to be electrically polarized in a certain biological microenvironment and which are also the main binding sites for nanoparticles. The charge on different cell surfaces does not only differ by its amount but also by its distribution. Unfortunately, it is difficult to measure this information directly using electric fields alone because electric fields experience 
significant interference when they go through the media and as a result direct physical contacts would be required, which makes the measurements difficult and costly.

Therefore, the underlying physics of our invention relies on using magnetoelectric nanoparticles (MENs) to convert the electric charge information stored on the cell surface into a certain magnetic field pattern which could be quickly measured by external magnetic measurement setups such as NMR, VSM, MOKE magnetometry, B-H looper, or others.

\subsection{Device Principle}

The first embodiment would be in the form of a rapid NMR sweeper (it can be frequency or magnetic field sweep). The schematic in Figure 9 is not to scale but illustrates the basic components of the setup. The entire prototype apparatus in the lab is quite portable and would easily fit in a regular backpack. A more advanced embodiment would consist of a miniaturized battery-supplied integrated circuit chip, which could be purchased in a neighborhood pharmacy store. In the future, such an integrated chip for rapid bacteria/virus identification could be carried in a pocket or attached to everyday clothing; the chip would be wirelessly connected to a computing device such as a smart phone, watch, laptop, or another, which in turn would provide the constantly updated database of spectra for different bacteria and viruses; such a database could be shared through the internet. 


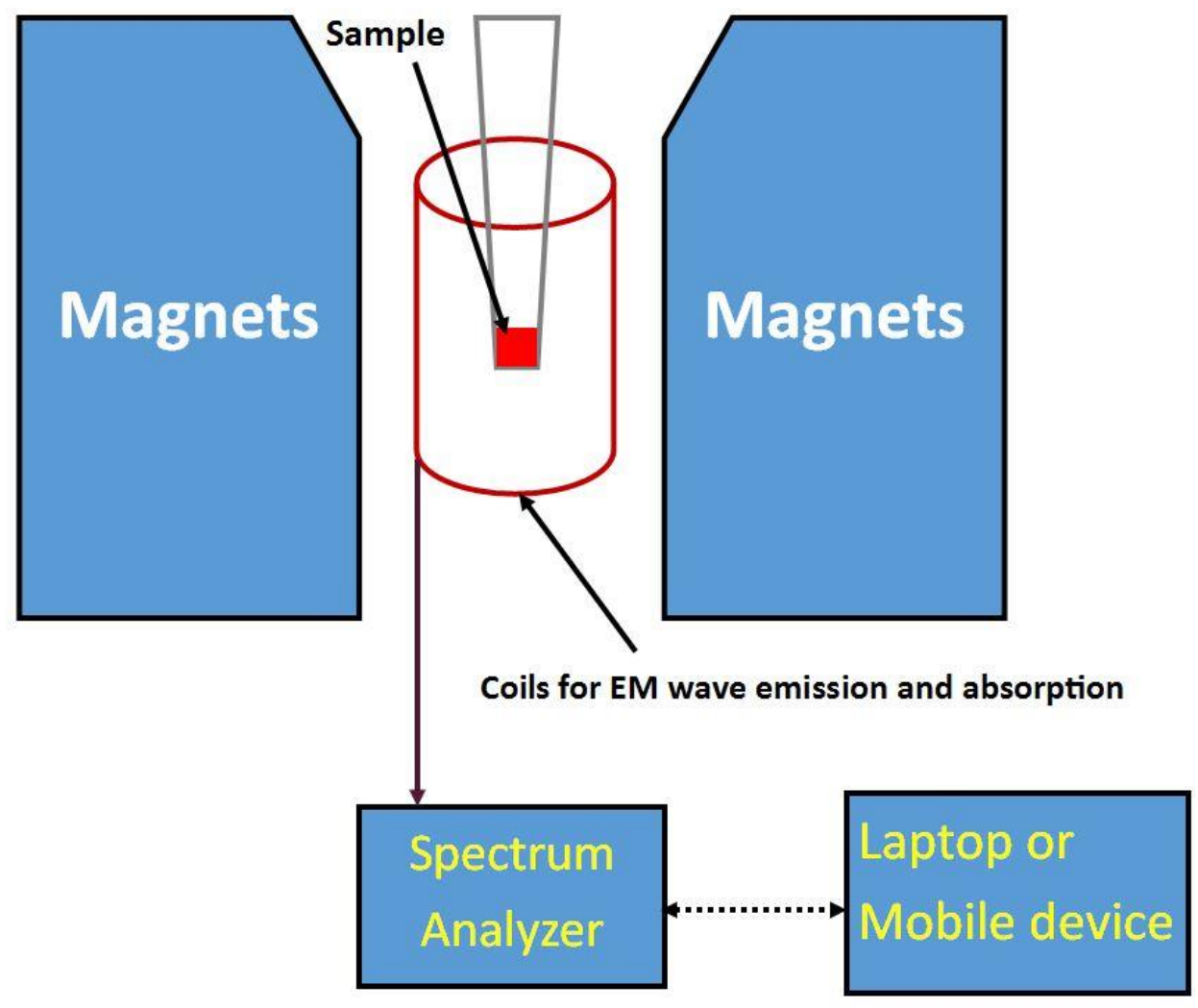

Figure 9: A basic device implementation for rapid screening of bacteria, viruses, and other cellular microorganisms.

The current device is quite portable in that it will fit inside a regular bag or backpack. A future device implementation would be in the form of an integrated microchip, which could be carried in a pocket or integrated in everyday clothing. In this case, a patient sample under study would optionally emerged be integrated into a special media, with the nanoparticles uniformly distributed in the media, to enhance the charge accumulation on specific surface sites characteristic of the sample. For example, in case of a virus, it could be put in a special stabilizing solution (which would also preserve the integrity of the sample) with a neutral physiological $\mathrm{pH}$, such as Tris-EDTA buffer solution, PBS, or 
another, to enhance the charge presence at low-dimensional irregularities (protruding proteins, receptors, etc.) on its surface where the charge would be more strongly accumulated (localized) to reflect the signature characteristics of this particular virus (Figure 10).

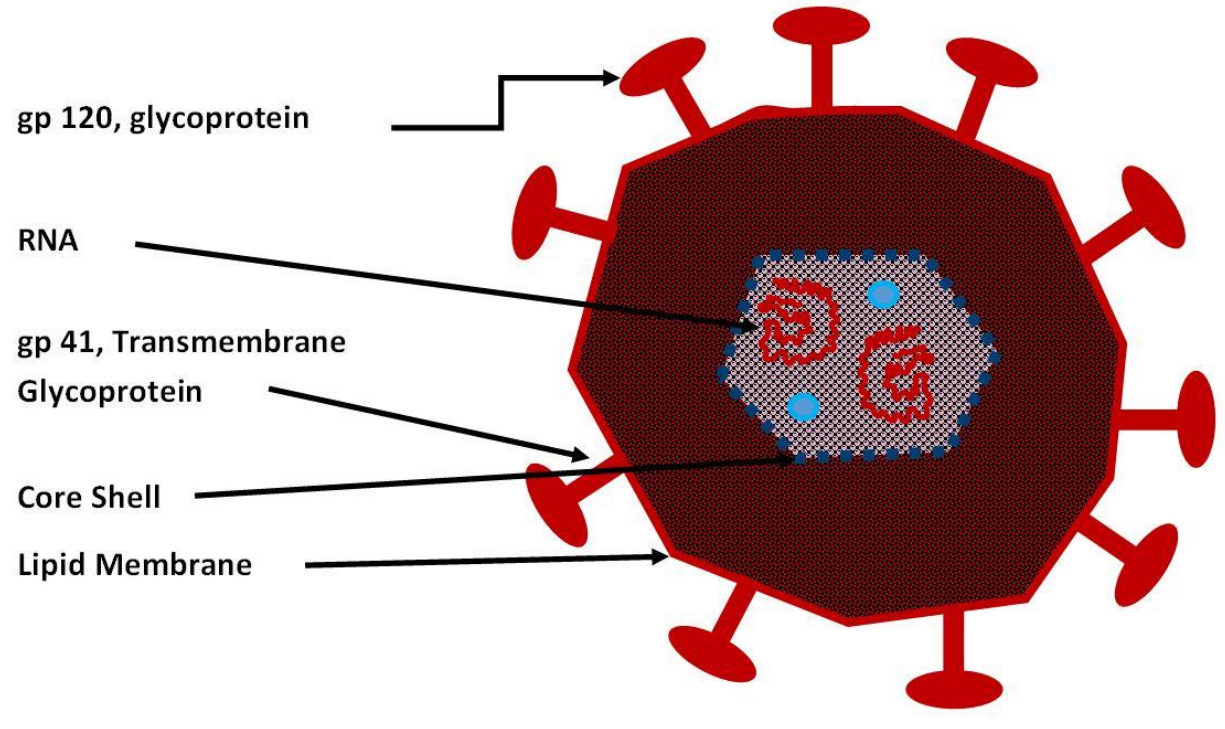

Figure 10: Schematic illustration of a HIV virus with characteristic protruding proteins/ receptors. Nanoparticles bind to these proteins.

As the nanoparticles bind to the characteristic surface sites, the nanoparticles are subjected to local electric fields due to the signature charge accumulations. Because of the unique magnetoelectric (ME) coefficient, these local electric fields generate local magnetic fields, which in turn superimpose with the magnetic field generated by the NMR setup. The NMR signal, i.e. the electromagnetic energy absorption, is generated when the resonance condition, $h v=M_{n} H_{n e t}$ is satisfied, where $h$ is the Plank constant, $v$ is the frequency of the electromagnetic wave (usually in $\mathrm{kHz}$ range in the NMR setup), $M_{n}$ is the 
nuclear spin measured in NMR (usually, proton spin), $H_{\text {net }}$ is the net magnetic field. This local net field is made of the two contributions, (i) the external field generated by the NMR magnets, $\mathrm{H}_{0}$, and contribution due to the ME effect of MENs on the surface of the virus, $H_{M E}$, respectively: $H_{n e t}=H_{0}+H_{M E}$.

As a result, each NMR spectrum will reflect the surface charge contribution of specific cells. Examples using bacteria, virus, and blood cells are presented in the proceeding chapters.

To confirm the underlying mechanism of the nanoparticles binding to specific sites on viral and bacterial surfaces, the following atomic force microscopy (AFM) experiments were conducted with several different strains of bacteria (Figure 12 through 15). AFM directly measures the surface topography with a nanometer spatial resolution. It could be mentioned that the viral surfaces display even more pronounced differences and therefore would result in a more significant effect. However, for the sake of simplicity, we first focused on the bacteria, which were currently available in our laboratory.

The respective NMR spectra measured for different bacteria strains are shown in Chapter 4. Two embodiments of MENs, uncoated nanoparticles, and nanoparticles functionalized with a thin layer glycerol monooleate (GMO), respectively, are shown.

As embodiment complementary application, the setup can be used to identify different cell types, including normal and cancer cells of the same or different types at different cancer progression stages. The physical origin of the charge accumulation on the 
surface is somewhat different between viruses and cells since viruses lack sophisticated membrane structure like that found on cells. In addition to the preferred binding sites due to specific proteins/receptors/etc., the accumulated charge strongly depends on the electric charge capacity of the cell membrane. For example, the charge stored on the cancer cell membrane is significantly smaller than that on the normal cell membrane of identical type. Additionally, the capacity depends on the cancer progression stage. Therefore, the charge contribution of the cell membrane is complex and made of several components.

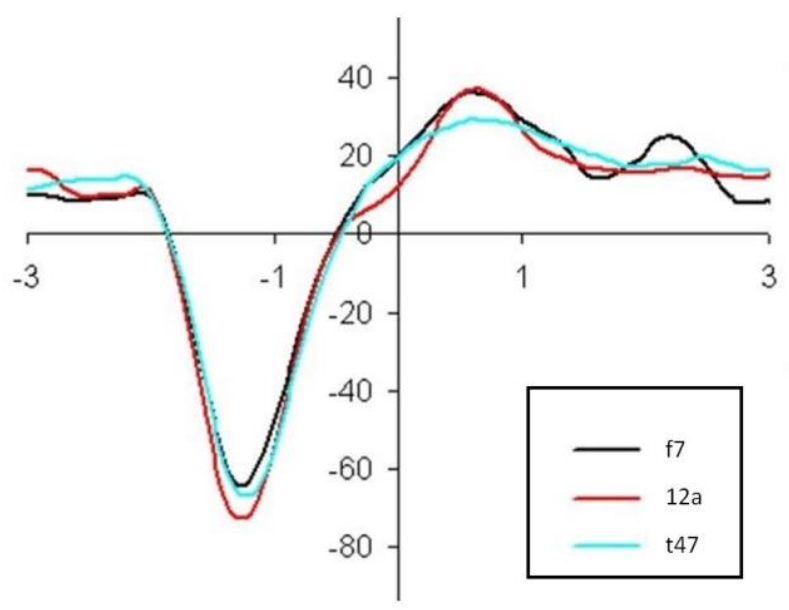

Figure 11: Spectra of normal cell line (red), cancer cell line f7 (black) and t47 (green)

As an example, we used our biosensing technology to measure the spectra of three cell lines including a normal cell line (red line 12a) and two cancer cell lines f7 and t47 at different progression stages, as shown in Figure 11. Clearly, the spectrum for the normal cell line has a distinctly different parabolic dependence compared to the cancer cell lines. 
In turn, the two cancer cell lines are different quantitatively. Experimental design and measurement is described in detail in Chapter 5. 


\section{SIGNATURE IDENTIFICATION OF BACTERIAL SAMPLE}

\subsection{Background}

Rapid and accurate identification of microorganisms like bacteria is critical for improving the state of care of infectious diseases. Lack of rapid identification and therapeutics of bacterial infection epidemics has a significant growth in recent times. As described in chapter 1.2 , recently developed lab-on-a-chip technology that can detect the traces of certain bacteria and viruses has made it possible for rapid diagnostics and testing in the field, although it still requires testing in a special biosafety facility. The traditional screening approach is to use bacterial culture and biochemical staining. This technique, unfortunately, requires a tedious sample preparation protocol and is relatively slow (a matter of hours and sometimes days), and is limited to a few biomarkers. On its north, an advanced approach based on polymerase chain reaction (PCR) is exorbitant to be used in the most clinics and small office environments. In order to solve the problem, our group has designed and investigated a device as, described in chapter 3 , for sensitive and rapid screening for various biological entities such as bacteria, viruses, cancer cells, and other biomolecular structures. Over the past several years, we concocted this device on the principle of a small package and low-cost in order to make it readily available even in the poorest healthcare facilities. This device is based on continuous wave nuclear magnetic resonance (NMR) technology.

\subsection{Sample preparation}

First, we used bacterial samples to get establish their signature signals using NMR. LB media plates were streaked with frozen culture stock and incubated overnight. Single colony inoculated LB media was incubated overnight on a shaker. Cells were washed with 
and resuspended in saline. Optical density was adjusted and normalized with saline to achieve an OD600 of 1x or 3x. Bacteria was incubated with MENs for 30 minutes at room temperature on a rotator. Specific amounts used for each experiment are listed beside results. Samples were prepared in triplicates. We used TEL cws 12-50 Advanced NMR/ESR System and its accompanying software to sweep the field or the frequency. Glass capillary tubes delivered the solution into the reader. The $\mathrm{B}_{0}$ was initially adjusted with a MENs only solution before any measurements were taken, and was also periodically adjusted as needed to mitigate expected shift in between readings. The graphs presented are averages of the 3 samples used.

A triplicate of each Pseudomonas aeruginosa, Escherichia coli, Serratia marcescens, and Bacillus cereus was used for each experiment and the same samples were used for to prepare slides for Atomic Force Microscope (AFM) and Scanning Electron Microscope (SEM) imaging. These bacterial strains were selected to demonstrate a variety of possible pathogens that could be detected with this system.

The AFM images shown in Figure 12 through Figure 15 shows the surface morphology of pseudomonas aeruginosa, escherichia coli, serratia marcescens, and bacillus cereus respectively. Top two figures are images of samples without nanoparticles while the bottom two are with particles. The left side images are the height maps whereas the right ones are the amplitude. The height maps on the left do not really look like the real bacteria as it appeared in the SEM images and such images do not display easily the shape of the features. Ways around this include shading the image, and more commonly, creating a pseudo-3D image from the height data. However, the deflection (or 
amplitude) images are equivalent to a map of the slope of the sample, so they often display the shape of the sample more clearly. The z-scale in deflection or amplitude is completely meaningless in terms of the sample structure. All it shows is how the tip deflected as it encountered sample topography. Nevertheless, the AFM images could not clearly show the topography of binding MENs to the surface of the bacteria. The $10 \mu \mathrm{m}$ scale AFM image does not show good MENs binding with the bacteria due to low resolution and sensitivity. Some bacteria like bacillus cereus may not interact strongly with the MENs. SEM images (Figure 16 through Figure 19) shows better clustering of particles with bacteria.

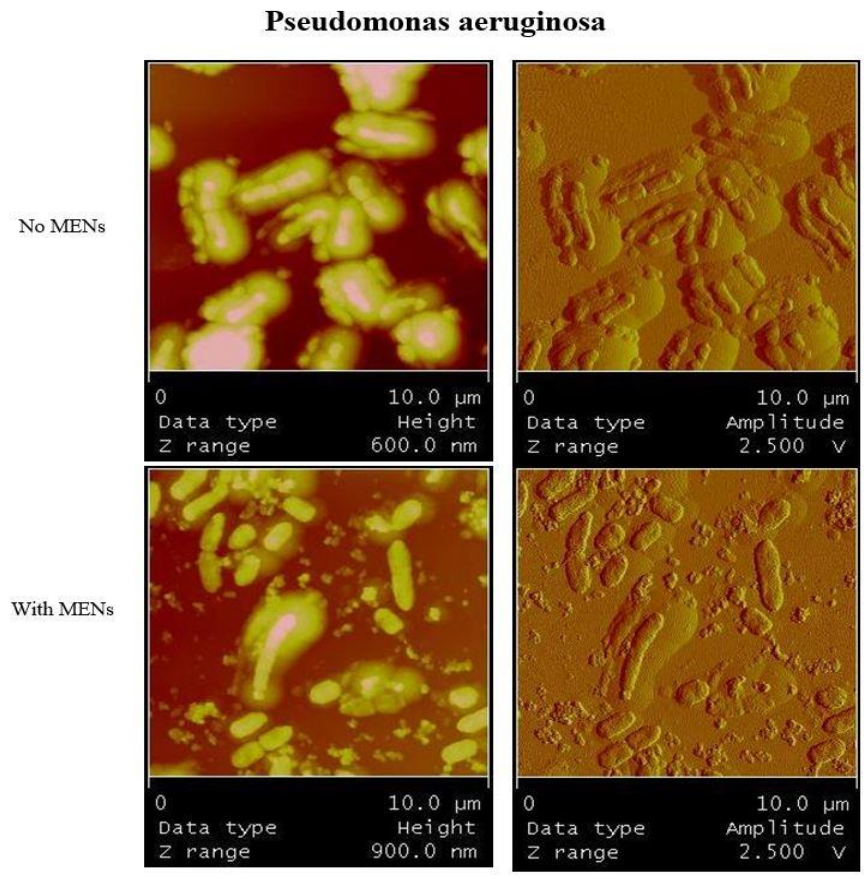

Figure 12: AFM image of Pseudomonas aeruginosa in a medium without MENs (top two) and with MENs (bottom two) 


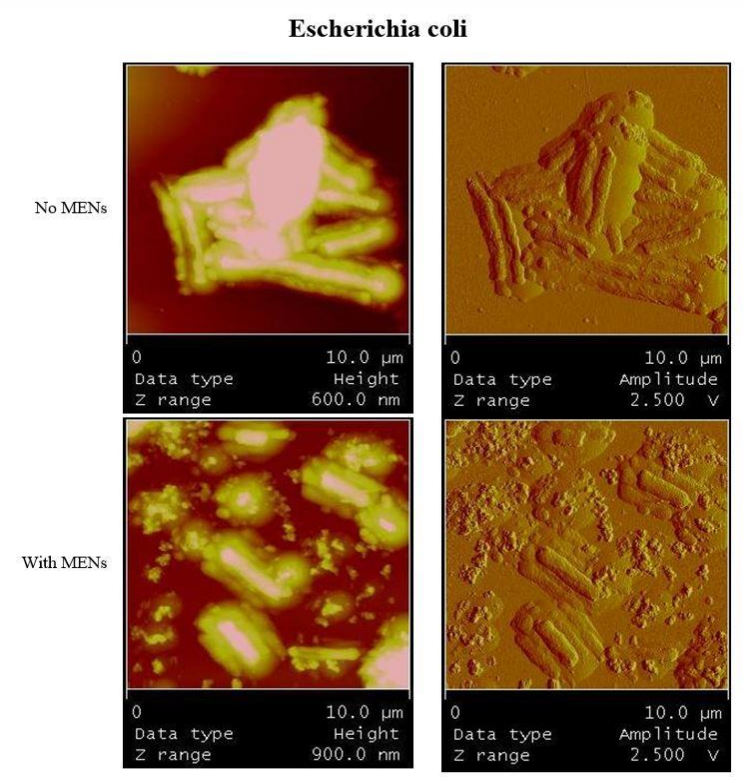

Figure 13: Figure 3.5: AFM images of Escherichia coli in a medium without MENs (top two) and with MENs (bottom two)

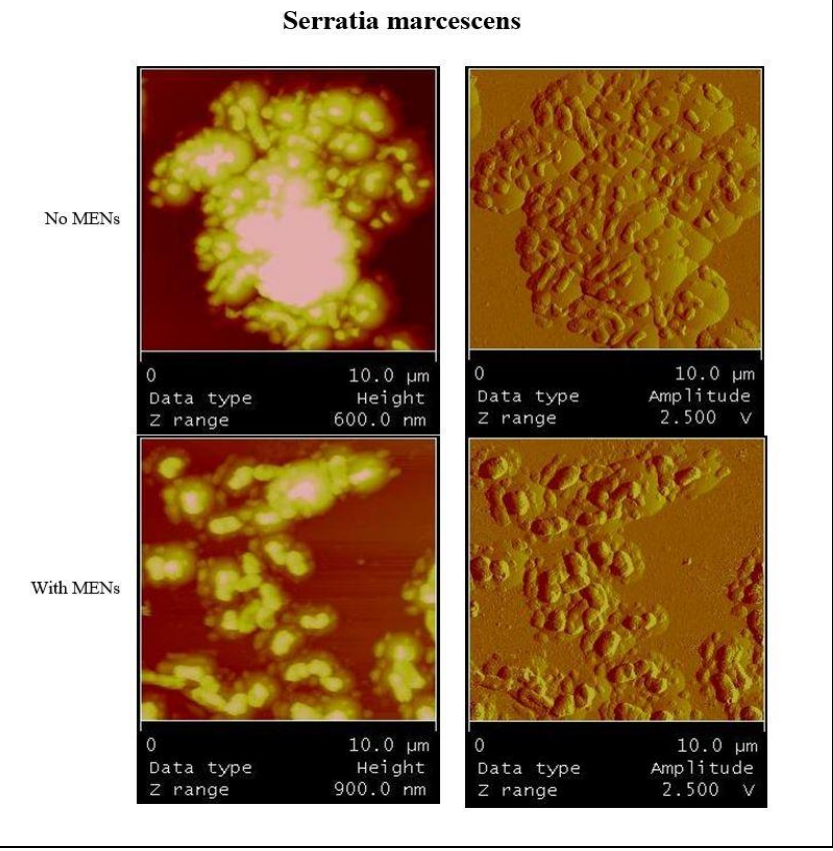

Figure 14: AFM images of Serratia marcescens in a medium without MENs (top two) and with MENs (bottom two). 


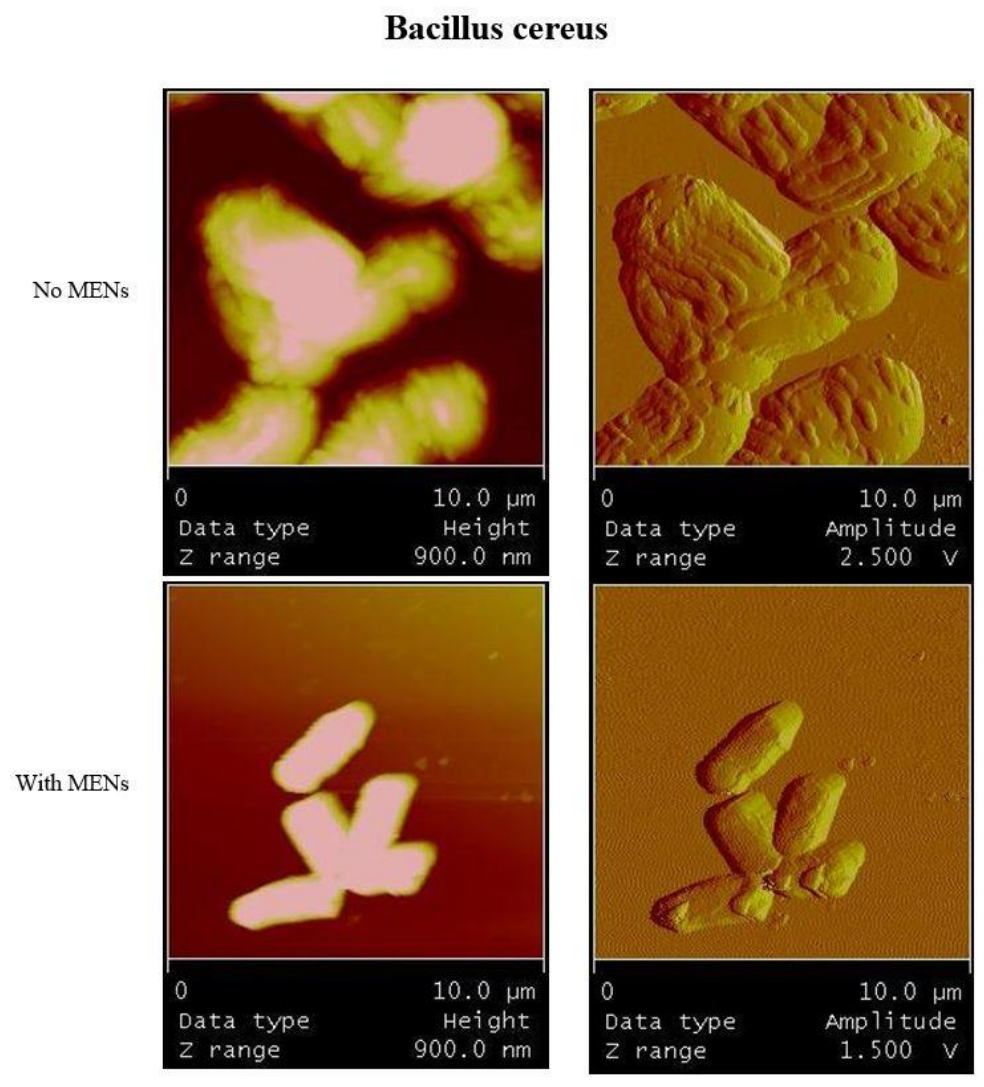

Figure 15: AFM images of Bacillus cereus in a medium without MENs (top two) and with MENs (bottom two).

The following SEM images Figure 16 through Figure 19 shows the clustering of MENs with the bacteria. The SEM images shown in Figure 16 through Figure 19 show the surface morphology of pseudomonas aeruginosa, escherichia coli, serratia marcescens, and bacillus cereus respectively. Top two figures are images of samples without nanoparticles while the bottom two are those with particles. The left side images are with the lower magnification and the right ones are with higher magnification (resolution) as shown in the labels at the bottom bars of each images. 

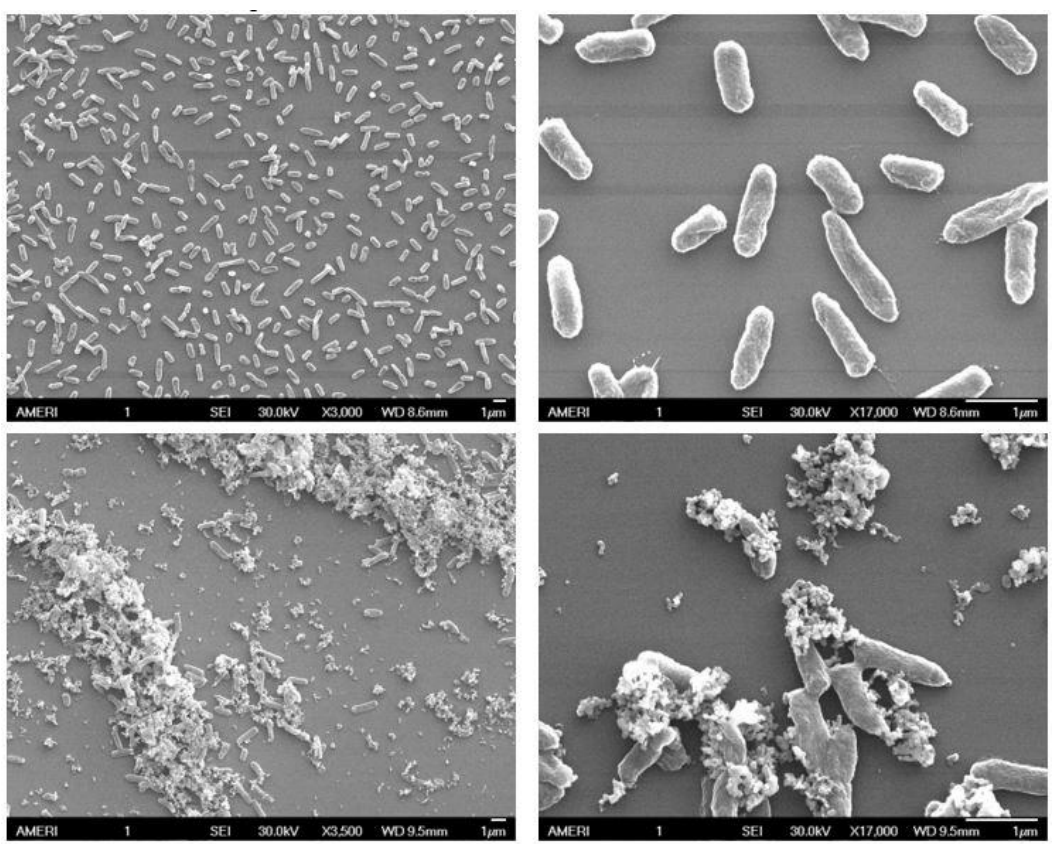

Figure 16: SEM images of pseudomonas aeruginosa without MENs (top two) and with MENs (bottom two) with different magnifications as shown in the label.
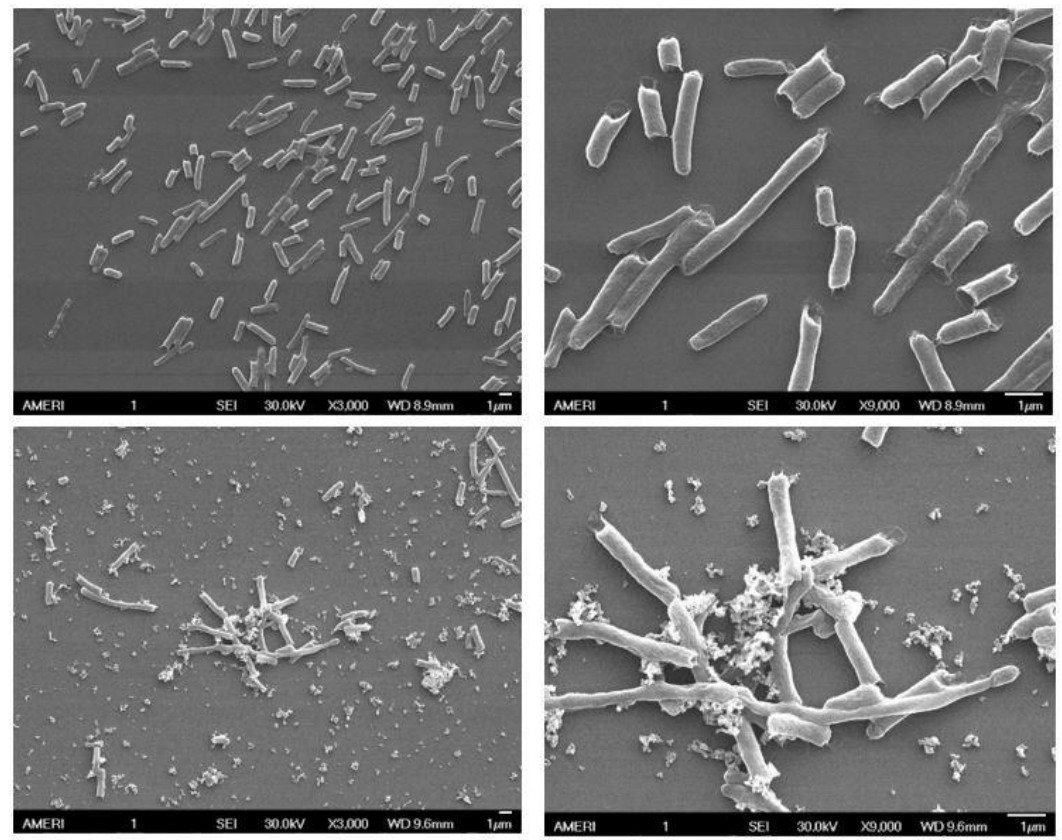

Figure 17: SEM images of escherichia coli without MENs (top two) and with MENs (bottom two) with different magnifications as shown in the label. 

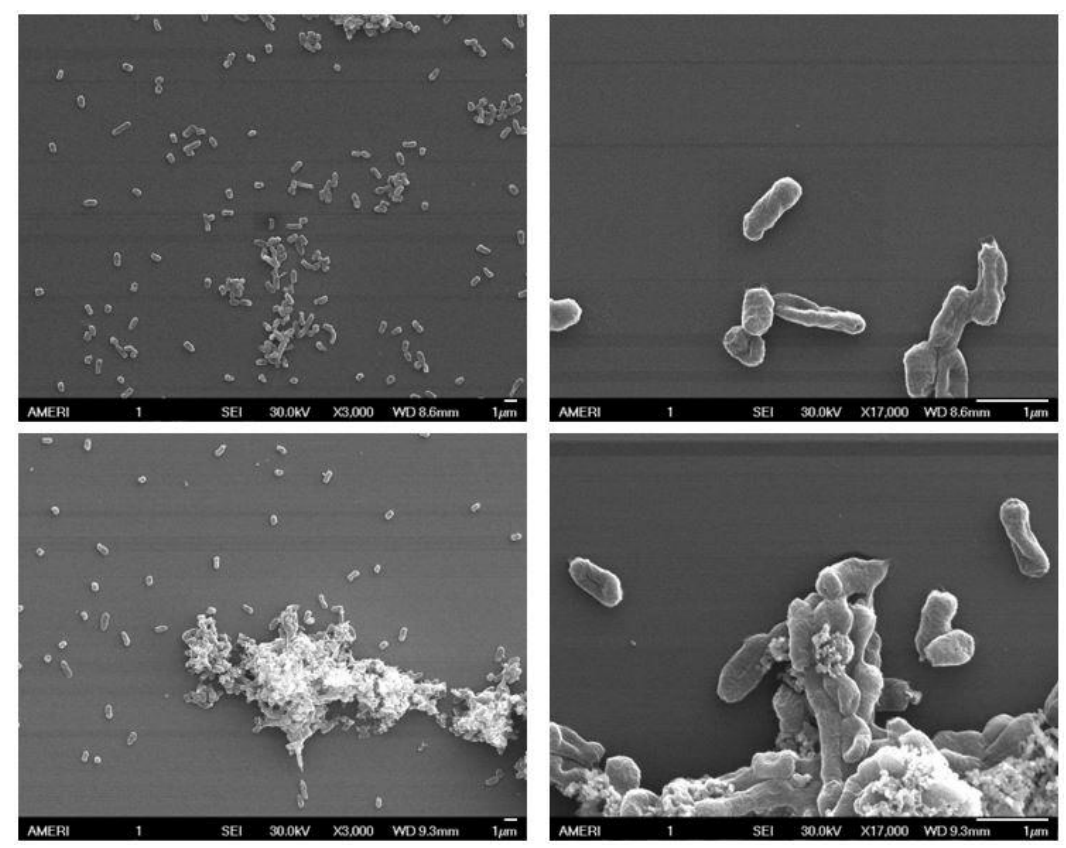

Figure 18: SEM images of serratia marcescens without MENs (top two) and with MENs (bottom two) with different magnifications as shown in the label.
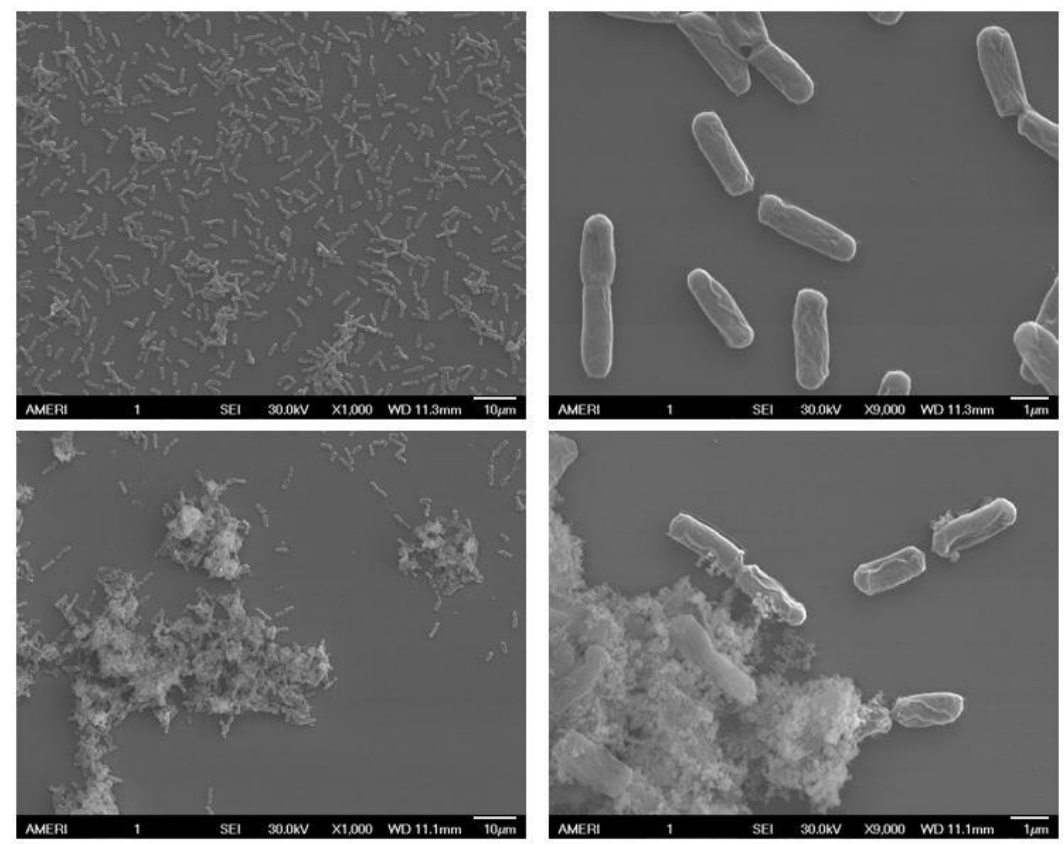

Figure 19: Figure 3.11: SEM images of bacillus cereus without MENs (top two) and with MENs (bottom two) with different magnifications as shown in the label. 


\subsection{Device optimization and bacterial measurement}

Device was designed as described in Chapter 3. The TEL cws 12-50 Advanced NMR/ESR System was optimized for the measurement of the bacterial sample. The absorbance of the sweep comes from the nanoparticles in the solution, meaning that a higher concentration of particles causes a greater absorbance (Figure $20 \mathrm{~A}$ ). The optimal concentration for testing was selected to be $50 \mathrm{mg} / \mathrm{ml}$ as it resulted in the largest increase in absorption coupled with the smallest standard deviations. The saline solution and bacterial cells give only a very weak background signal (Figure $20 \mathrm{~B}$ ).

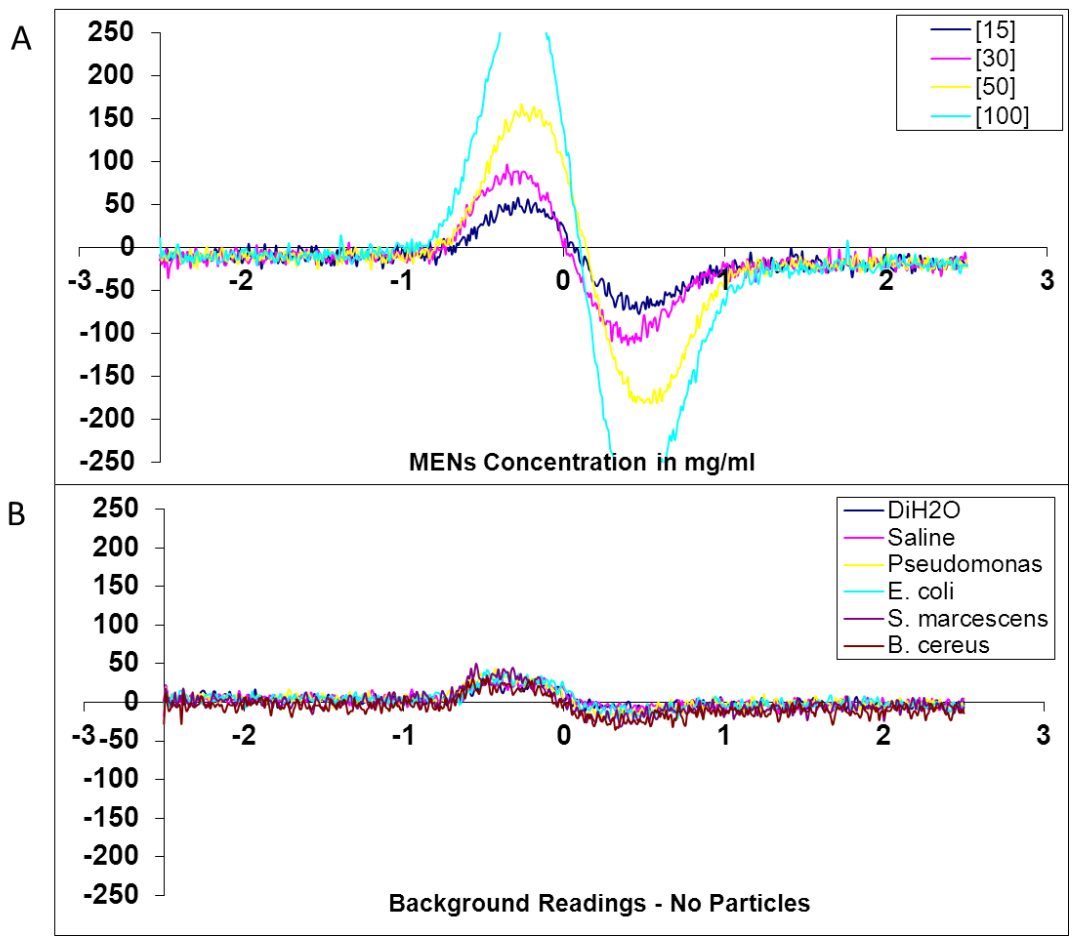

Figure 20: NMR Absorbance. A) Nanoparticles in the solution determine the strength of the absorbance. B) Bacterial solutions have a very weak absorbance. 


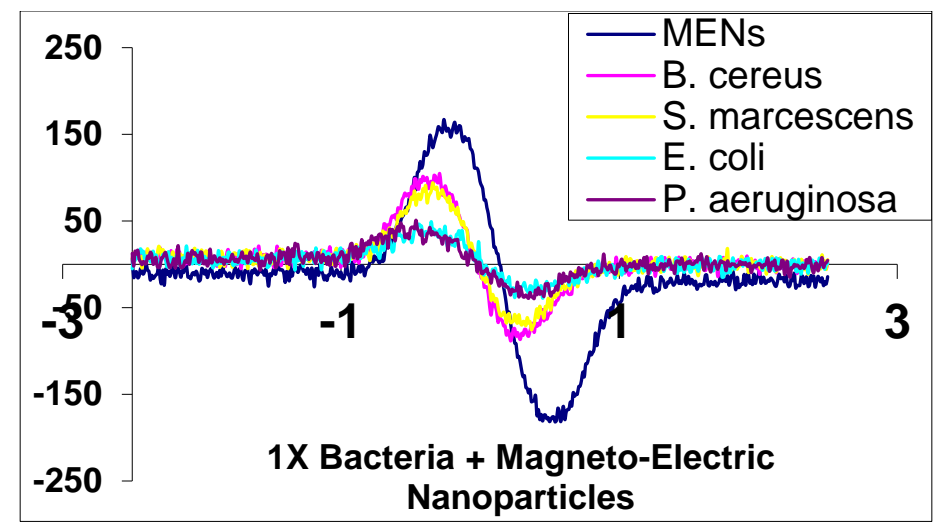

Figure 21: Pelleted $1 \mathrm{ml} 1 \mathrm{X}$ bacteria, suspended in $20 \mu \mathrm{l}$ saline, added $20 \mu \mathrm{l} 50 \mathrm{mg} / \mathrm{ml}$ MENs.

Next, the optimal phase for the field sweep was determined to be around 300 (Figure 22) and 297 was used for all readings. MENs were determined to the superior identifier of bacteria compared to magnetic nanoparticles and GMO-coated MENs (Figure 23). Frequency sweep results confirm patterns seen in the field sweep used (Figure 24). 


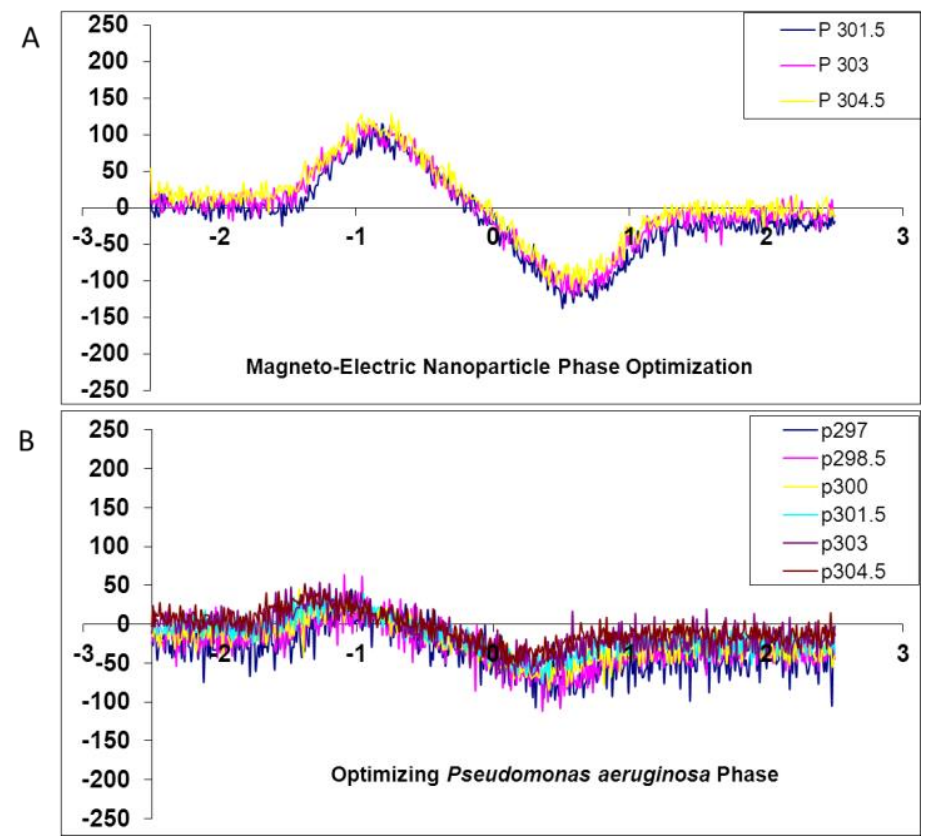

Figure 22: Phase Optimization for MENs. A) MENs signal is strongest around a phase of 300. B) An example of a solution of bacteria with no MENs showing weak signal even at optimized settings.

A

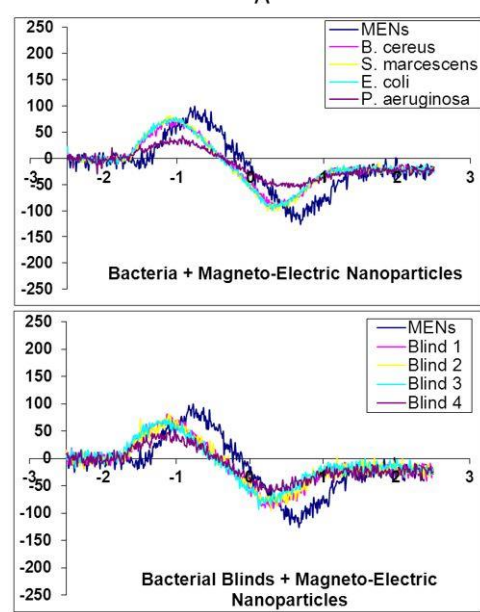

Figure 23: MENs Provide Best Blind Differentiation. A) MENs being used to identify blinds. B) Traditional magnetic nanoparticles being used to identify blinds. C) GMO-coated MENs being used to identify blinds.

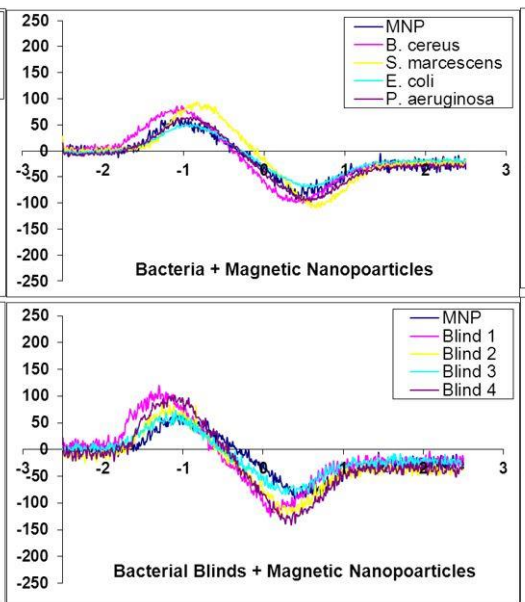

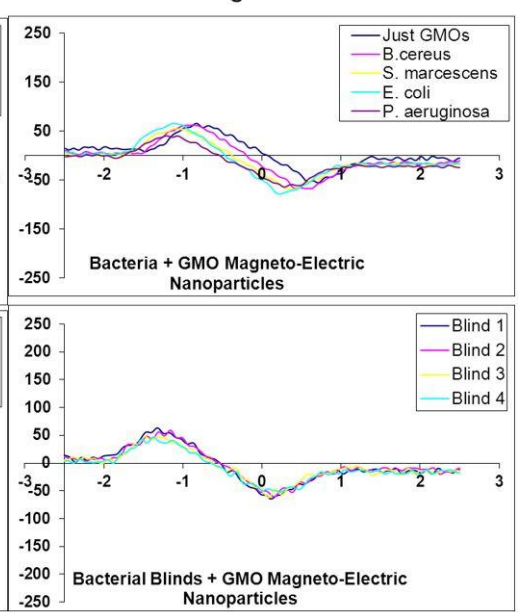

Nanoparticles 


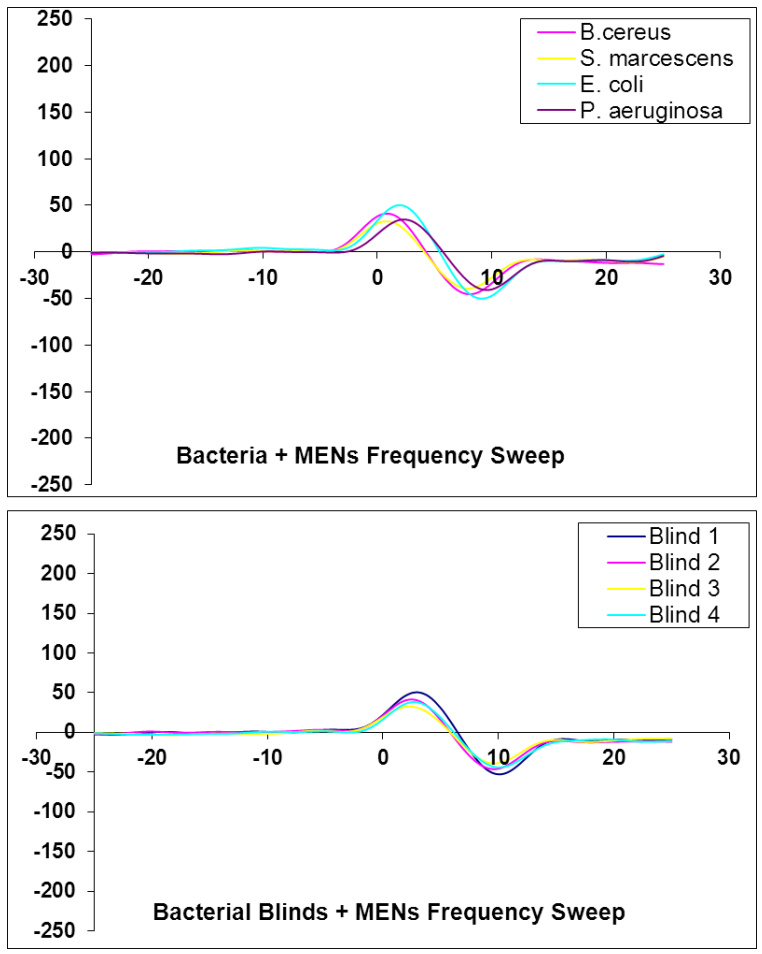

Figure 24: Frequency Sweep. The field sweep data used is consistent with the frequency sweep data, but is not better for identifying bacterial blinds. 


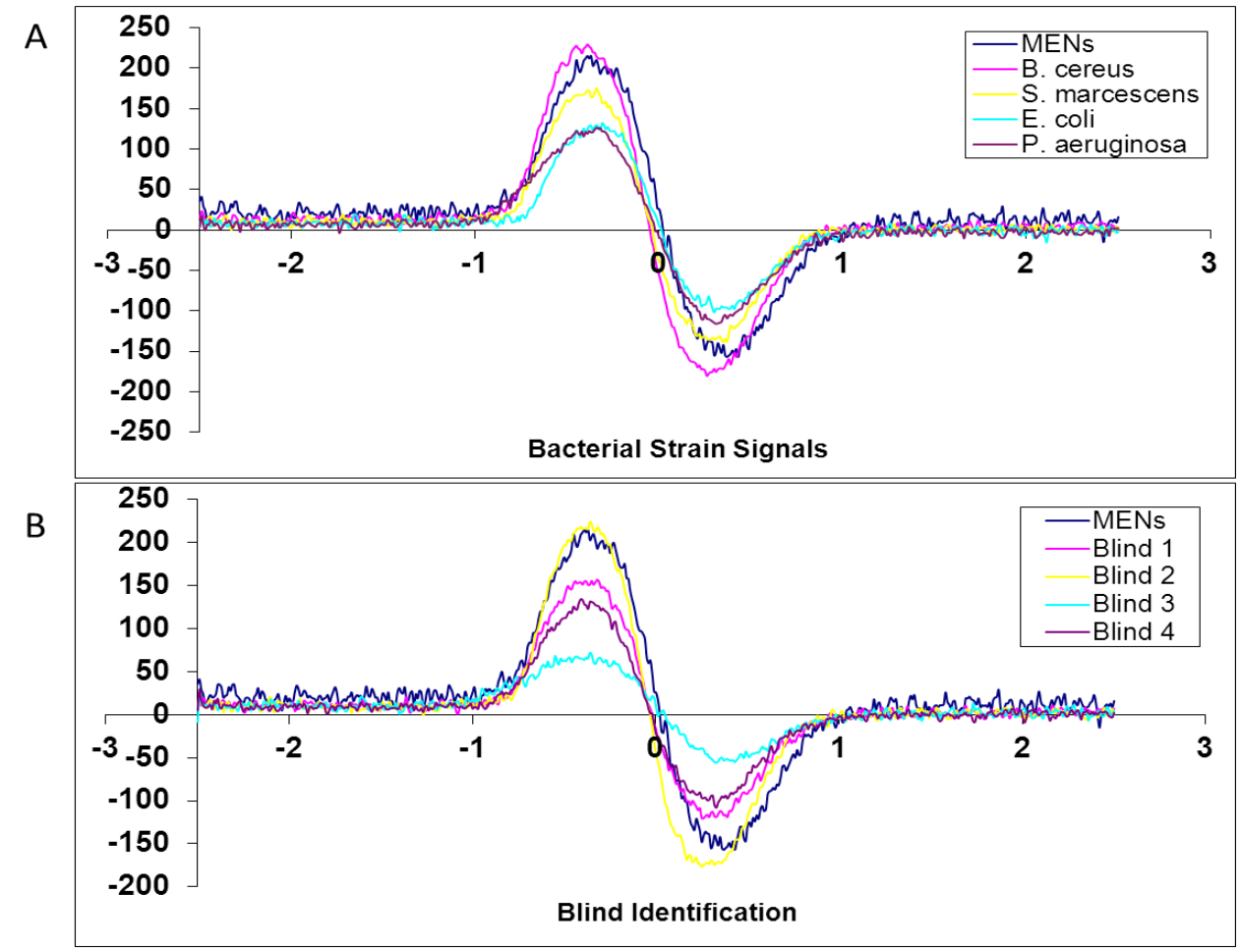

Figure 25: A) Four bacterial species were used to demonstrate the unique MENs interacting properties of each. B) Blind identities were successfully determined using the reference: $1=S$. marcescens, $2=B$. cereus, $3=P$. aeruginosa, $4=E$. coli.

Exploitation of intrinsic surface electromagnetic properties of biological organism works as a tool for biomedical signature and screening.

The direct curves were analyzed to plot the signature curves of all four bacteria using moving average fit curve. The background curve due to MENs only were removed to get the signature of the bacteria only. Following are the eyeball comparison of each bacterium with MENs and corresponding signature curve. 


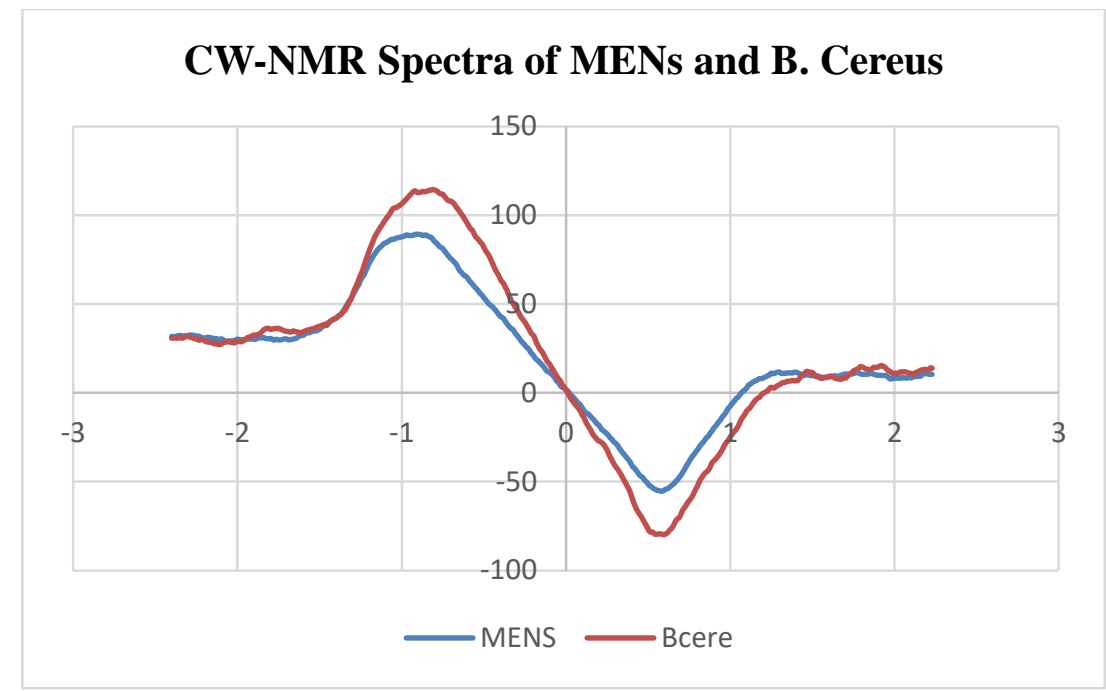

Figure 26: Eyeball comparison of MENs and Bacillus Cereus

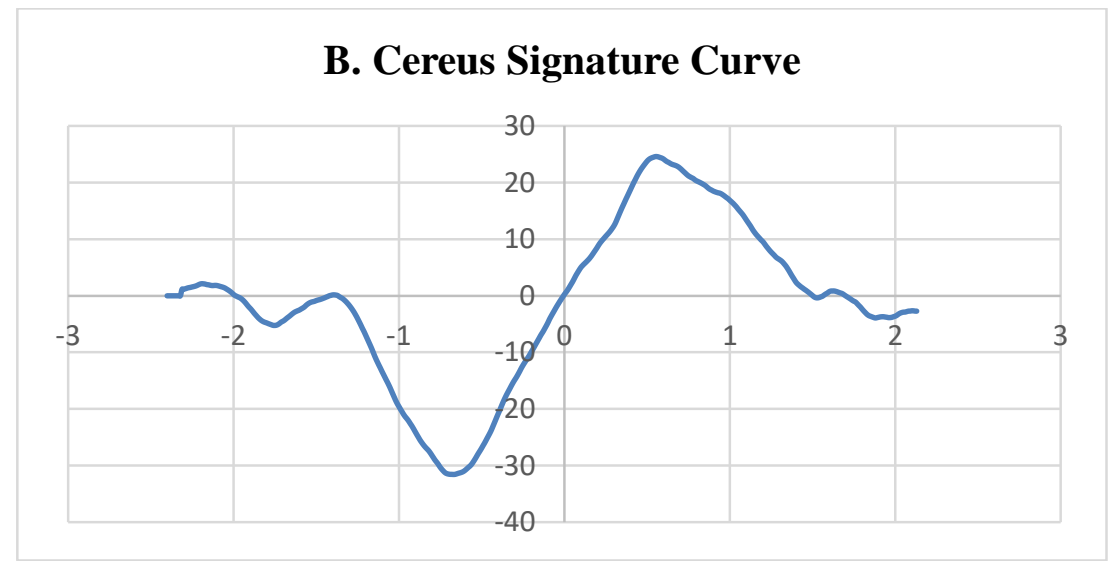

Figure 27: Signature curve of Bacillus Cereus 


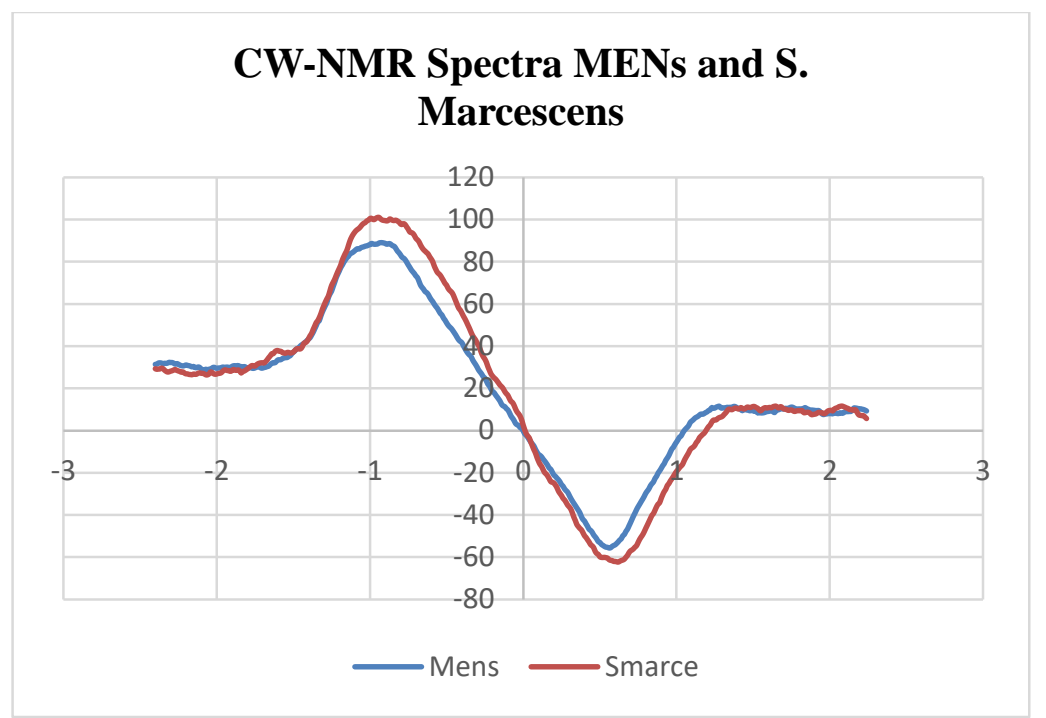

Figure 28: Eyeball comparison of MENs and Serratia marcescens

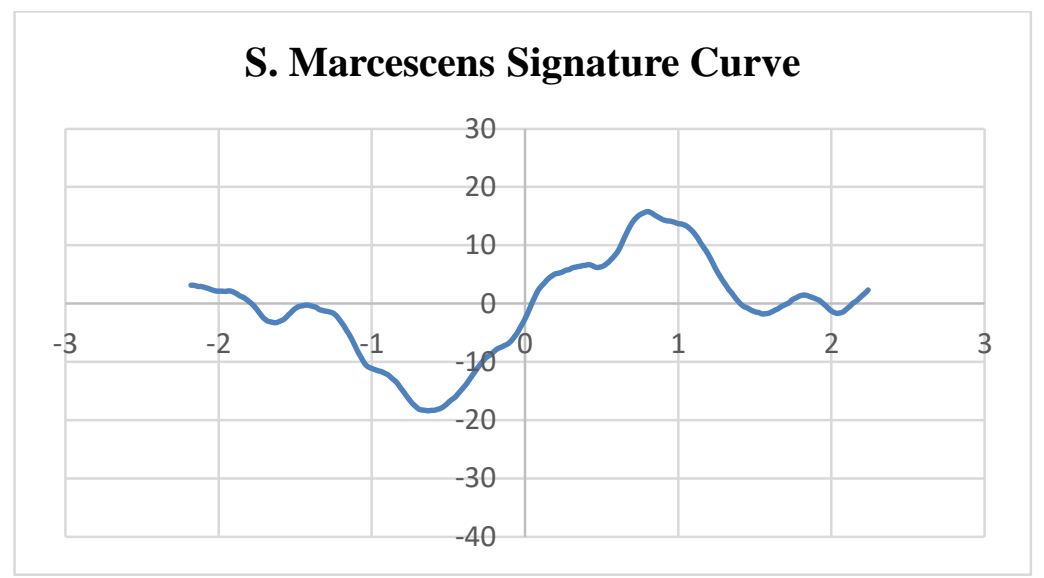

Figure 29: Signature curve of Serratia Marcescens 


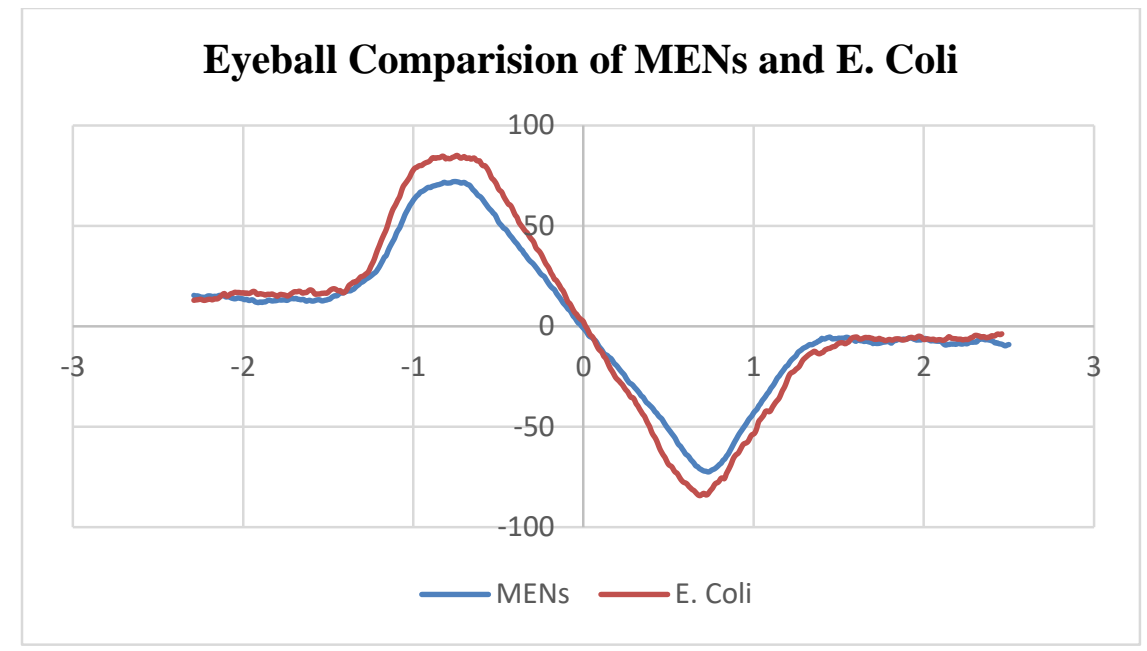

Figure 30: Eyeball comparison of MENs and Escherichia Coli

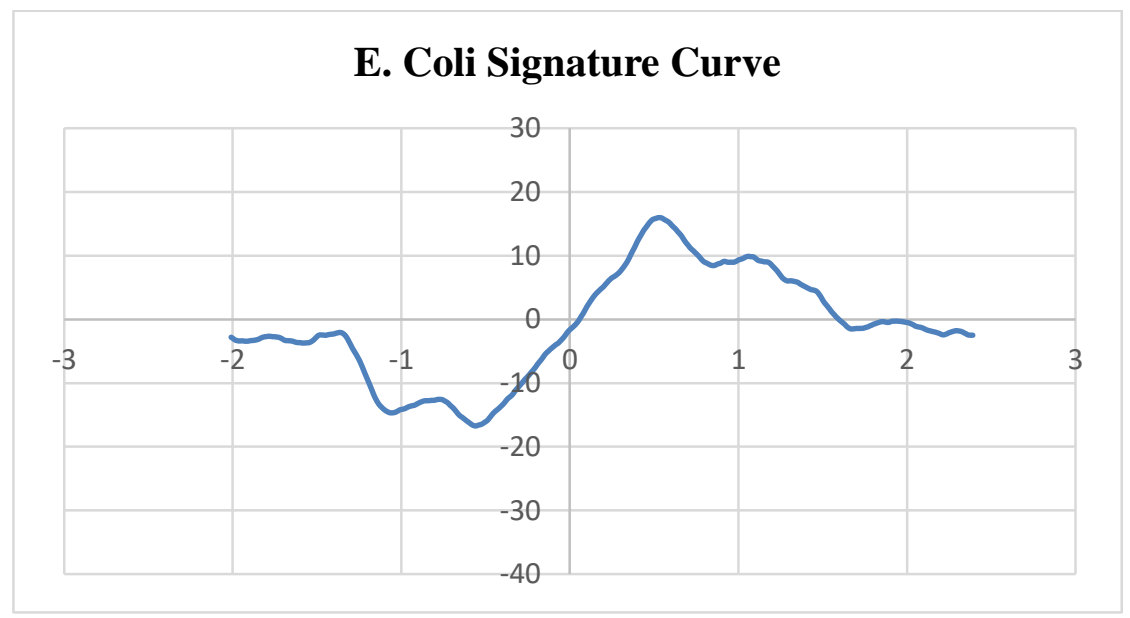

Figure 31: Signature curve of Escherichia Coli 


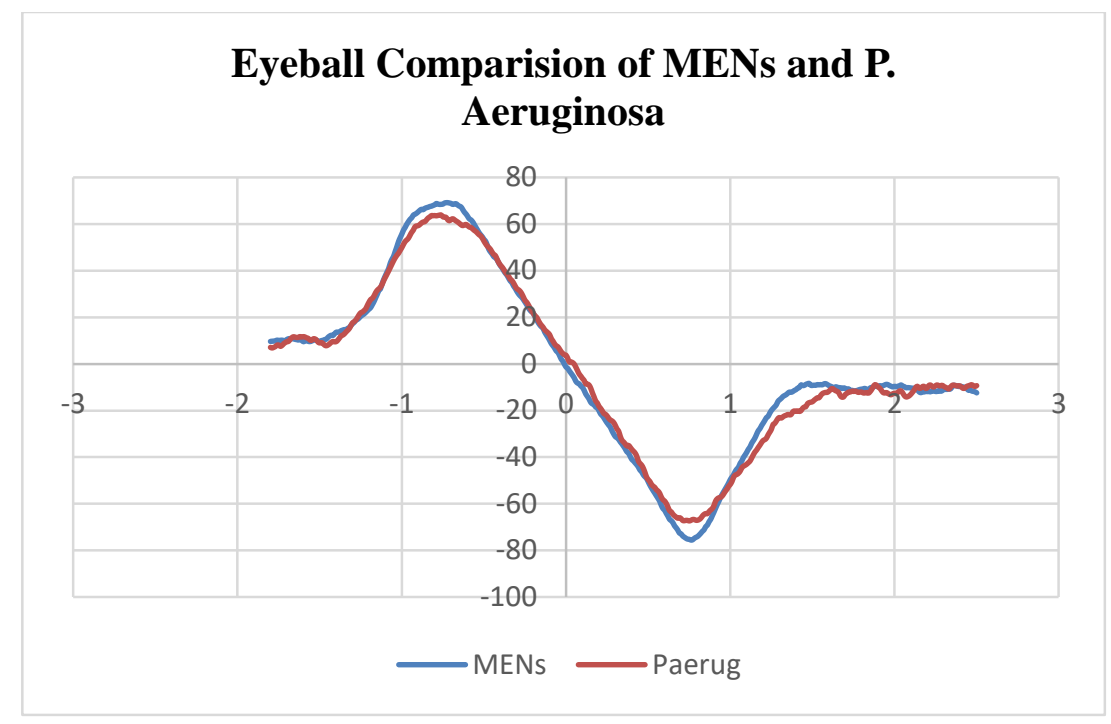

Figure 32: Eyeball comparison of MENs and Pseudomonas Aeruginosa

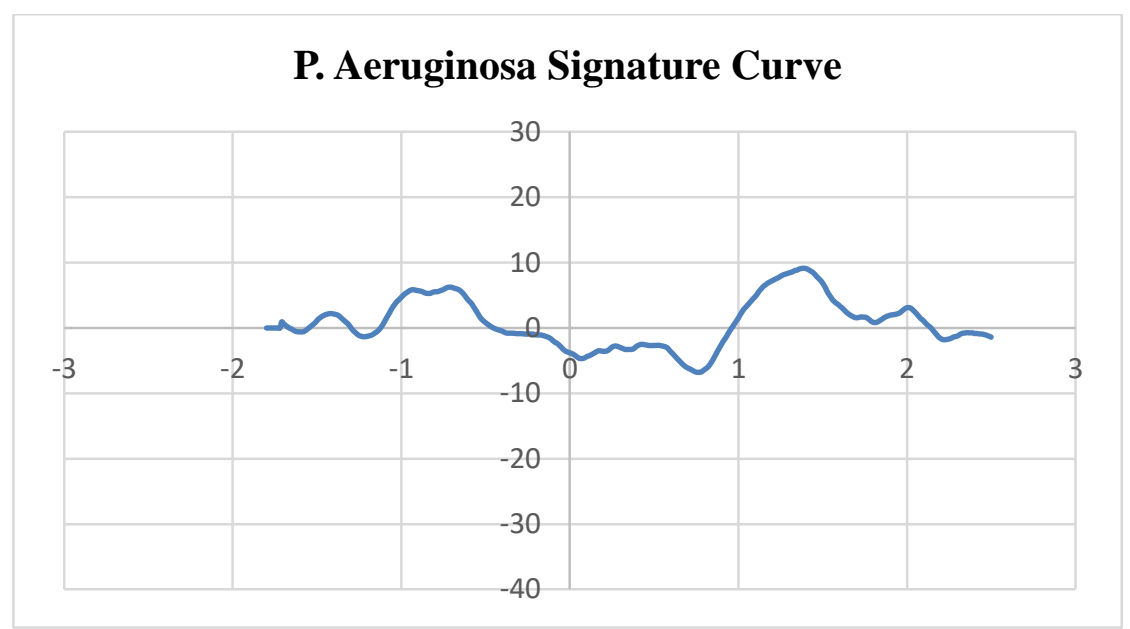

Figure 33: Signature curve of Pseudomonas Aeruginosa 


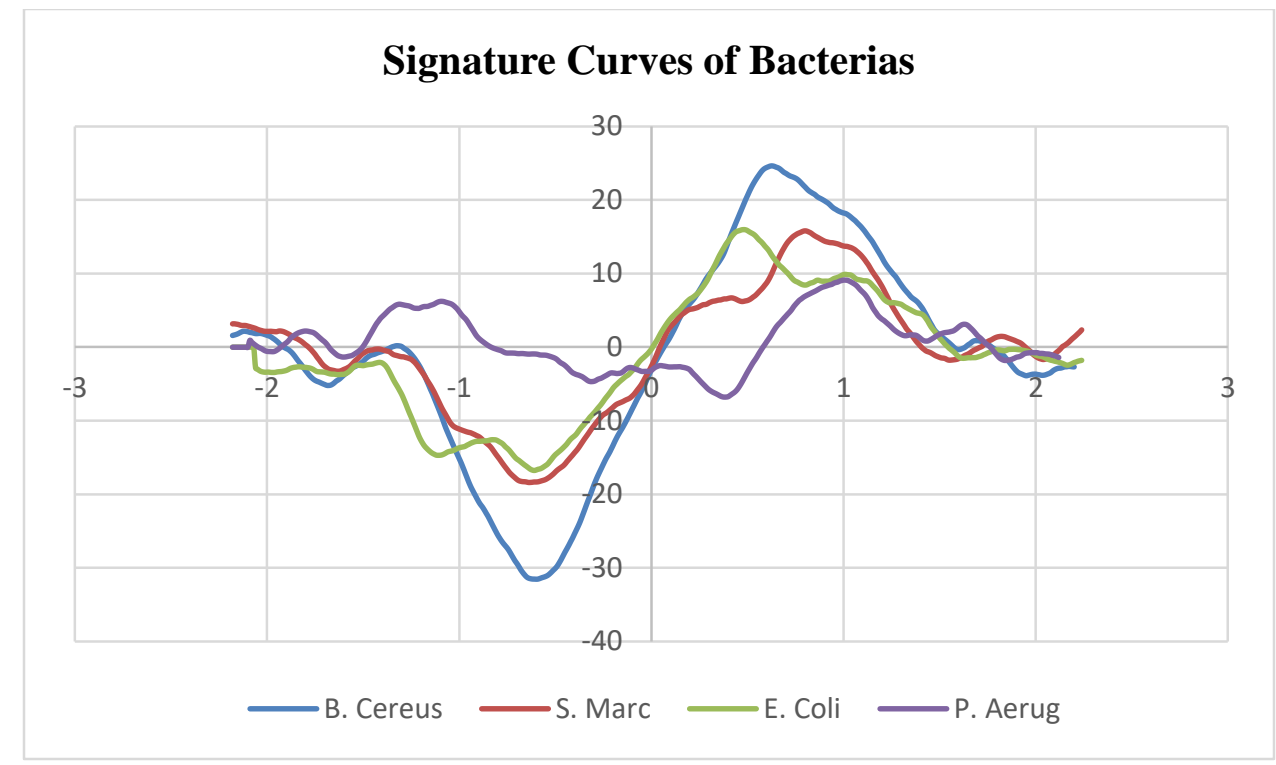

Figure 34: Signature curves of Bacillus Cereus (blue), Serratia Marcescens (red), Escherichia Coli (green), and Pseudomonas Aeruginosa (purple)

Signature curves of all four bacteria are unique. Although the signature curve of Serratia Marcescens (Figure 29) and Escherichia Coli (Figure 31) seems quite close but they are clearly distinguishable. Optimization and proper calibration of MENs concentration, sample preparation, precise measurement and proper signal processing application design will eliminate the doubt if any. The experiments on bacterial sample shows that the MENs could be used not only to distinguished between their counterparts but also with other types of cells. 


\section{SIGNATURE IDENTIFICATION OF CANCER CELL LINES}

\subsection{Background}

Rapid identification of cancer cells is vital for cancer prevention and treatment. Traditional techniques which rely on biochemical staining require a tedious sample preparation and are limited to a few biomarkers. A more advanced approach based on polymerase chain reaction (PCR) remains cost-ineffective in a small-clinic environment. Therefore, recently there has been increased interest in magnetic nanoparticle biosensing (MNB). Due to a new dimensionality provided by the presence of externally-controlled magnetic moments, MNB promises to enable high-specificity screening and fast diagnostic of pathogens. Indeed, ideally one could envision an apparatus in which magnetic nanoparticles are used to couple intrinsic information related to single cells, e.g. the electric charge profile on the surface of a cell in a specific biological microenvironment, to an external magnetic device such as a nuclear magnetic resonance (NMR) system. However, the current progress in this area still remains relatively slow. The main challenge is to couple magnetic nanoparticles to intrinsic information at the cellular or intra-cellular level with sufficiently high efficacy to be able to process the information with a magnetic system. While the system measures magnetic fields, the intrinsic cellular information is reflected in electric fields. It can be noted that in the cellular microenvironment, each cell structure, corresponding to a specific cancer type and a cancer progression stage, is characterized by a certain membrane surface morphology which in turn results in a signature electric-field configuration [65-67]. However, traditional purely magnetic nanoparticles wouldn't be able to detect this complex electric-field configuration, unless they have electric charges. To address this problem, in lieu of the traditional magnetic nanoparticles, we have used a 
type of multiferroic nanoparticles known as magnetoelectric nanoparticles (MENs). Unlike the traditional magnetic nanoparticles, MENs have both magnetic and electric dipole moments; in addition, these two different moments are correlated through the magnetoelectric (ME) effect. Due to the presence of an electric charge MENs preferentially attach to cell-specific sites, while due to the ME effect MENs allow to convert the intrinsic electric field information at the cellular level into a specific magnetic field pattern which in turn could be measured through a magnetic measurement setup such as an NMR system. Because each cell type has its own signature electric field distribution, such NMR measurements could be used to distinguish different cell types from each other at the intracellular level.

\subsection{Sample preparation and cell culture}

MENs were prepared as described in chapter 2.3. $\mathrm{CoFe}_{2} \mathrm{O}_{4}-\mathrm{BaTiO}_{3}$ core shell MENs were prepared according to the following conventional procedure. As the first step, $\mathrm{CoFe}_{2} \mathrm{O}_{4}$ particles were prepared by the standard hydrothermal method, according to which $0.058 \mathrm{~g}$ of $\mathrm{Co}\left(\mathrm{NO}_{3}\right)_{2} .6 \mathrm{H}_{2} \mathrm{O}$ and $0.16 \mathrm{~g}$ of $\mathrm{Fe}\left(\mathrm{NO}_{3}\right)_{3} .9 \mathrm{H}_{2} 0$ were dissolved in 15 $\mathrm{ml}$ of distilled water and $0.2 \mathrm{~g}$ of polyvinylpyrrolidone was dissolved in $5 \mathrm{ml}$ of aqueous solution containing $0.9 \mathrm{~g}$ of sodium borohydride at $120^{\circ} \mathrm{C}$ for 12 hours. Then, a precursor solution of $\mathrm{BaTiO}_{3}$ was prepared by mixing $30 \mathrm{ml}$ of aqueous solution containing $0.029 \mathrm{~g}$ of $\mathrm{BaCO}_{3}$ and $0.1 \mathrm{~g}$ of citric acid with $30 \mathrm{ml}$ of ethanolic solution containing $1 \mathrm{~g}$ of citric acid and $0.048 \mathrm{ml}$ of titanium (IV) isopropoxide. Coreshell $\mathrm{CoFe}_{2} \mathrm{O}_{4}-\mathrm{BaTiO}_{3} \mathrm{MENs}$ were prepared by mixing $0.1 \mathrm{~g}$ of $\mathrm{CoFe}_{2} \mathrm{O}_{4}$ nanoparticles in the $\mathrm{BaTiO}_{3}$ precursor solution and the mixture was sonicated for $2 \mathrm{hrs}$. Once the $\mathrm{CoFe}_{2} \mathrm{O}_{4}$ nanoparticles were thoroughly dispersed, the mixture was dried on the hot plate 
at $60^{\circ} \mathrm{C}$ overnight while being continuously stirred. The dried powder was heated to $780^{\circ} \mathrm{C}$ for $5 \mathrm{hrs}$. in a furnace (CMF-1100) and cooled at $52^{\circ} \mathrm{C}$ per minute to obtain the coreshell MENs of $\sim 30 \mathrm{~nm}$ diameter. The particles size distribution was measured using dynamic light scattering method (Malvern-Zetasizer).

Three cancerous cell lines Skov-3 (Ovarian adenocarcinoma), U87-MG (Glioblastoma), MCF-7A (Breast adenocarcinoma) and two non-cancerous cell lines viz Brain Endothelial cells (Brain EC) and Rat smooth muscle endothelial cells (RSMC) were cultured at $37^{\circ} \mathrm{C}$ as per manufacturer's instructions. For nanoparticle studies, cells were detached using $0.25 \%$ trypsin solution, plated in 6 well plates and allowed to grow to 80 $\%$ confluency. Magnetoelectric nanoparticles (MEN's) were resuspended in cell culture media through sonication and were incubated for 30 minutes. MEN's were added to each well at a concentration of $150 \mu \mathrm{g} / \mathrm{ml}$ and the cells were further incubated for 15 hours, to allow attachment of MEN's with the cells. Effect of d.c magnetic field was studied by exposing the cells incubated with MEN's to a magnet that was placed, at a distance, directly underneath the culture plate. The distance of magnet from cell culture plate needed to create 100 Oe field was determined using a gauss meter. U87 -MG cells were exposed to field for 6 hours after which the spectra was acquired whereas Brain EC's and RSMC's were exposed to field for 15 hours under MEN incubation. Additionally, all the cell lines were incubated with $150 \mu \mathrm{g} / \mathrm{ml}$ traditional $\mathrm{MNP}\left(\mathrm{CoFe}_{2} \mathrm{O}_{4}\right)$ for 15 hours with and without d.c. magnetic field. After the end of incubation period, the cells were washed with phosphate buffered saline (PBS) to remove particles not attached to cells. Cells were scraped from the bottom of plate and transferred to a $5 \mathrm{~mm}$ NMR tube. Continuous wave- ${ }^{1} \mathrm{H}$ NMR readings 
were obtained using a CWS 1250 NMR spectroscope. Sample placement, instrument parameters $\left(\mathrm{B}_{0}\right.$, instrument phase,line-width) were carefully selected to ensure optimal signal to noise ratio. NMR spectra was collected at opposite phases (in our case these were $107^{\circ}$ and $297^{\circ}$ ). The spectra from cells with/without MEN's was added with opposite phase signal of PBS to eliminate the solvent interference. The figures below are AFM images of endothelial cells (height on the left and phase on the right). Figure 35 (b) clearly shows that the MENs are inside the cells due to magnetic-electroporation when subjected to dc magnetic field as described earlier.
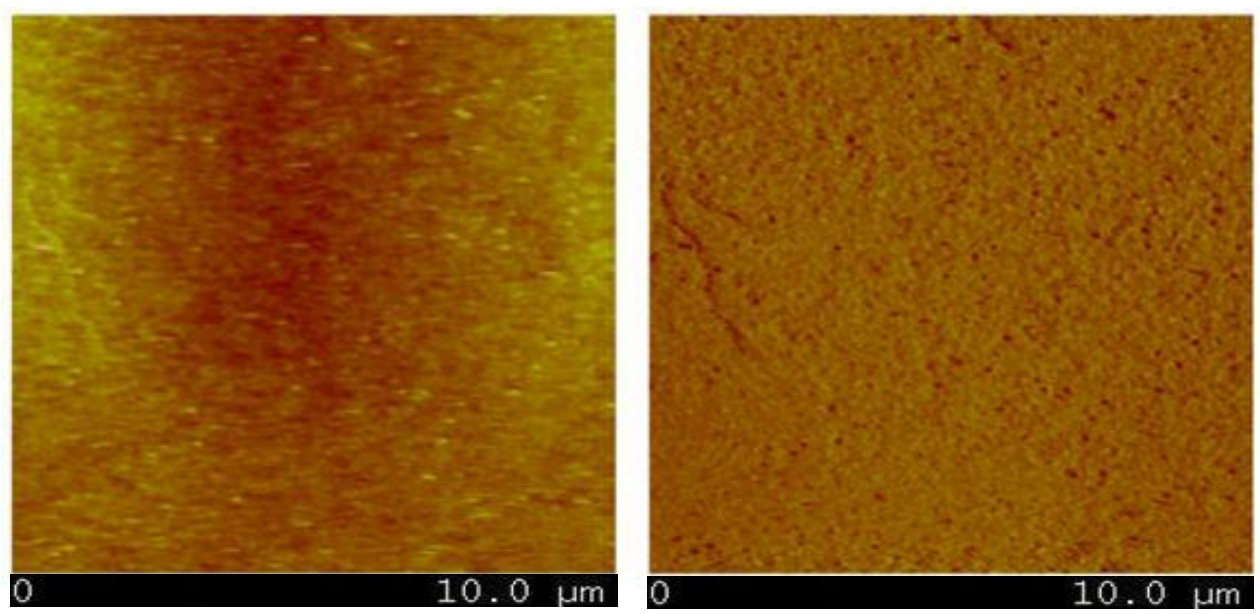

Figure 35: AFM image pair of endothelial cells (z-height (left), phase (right)) 

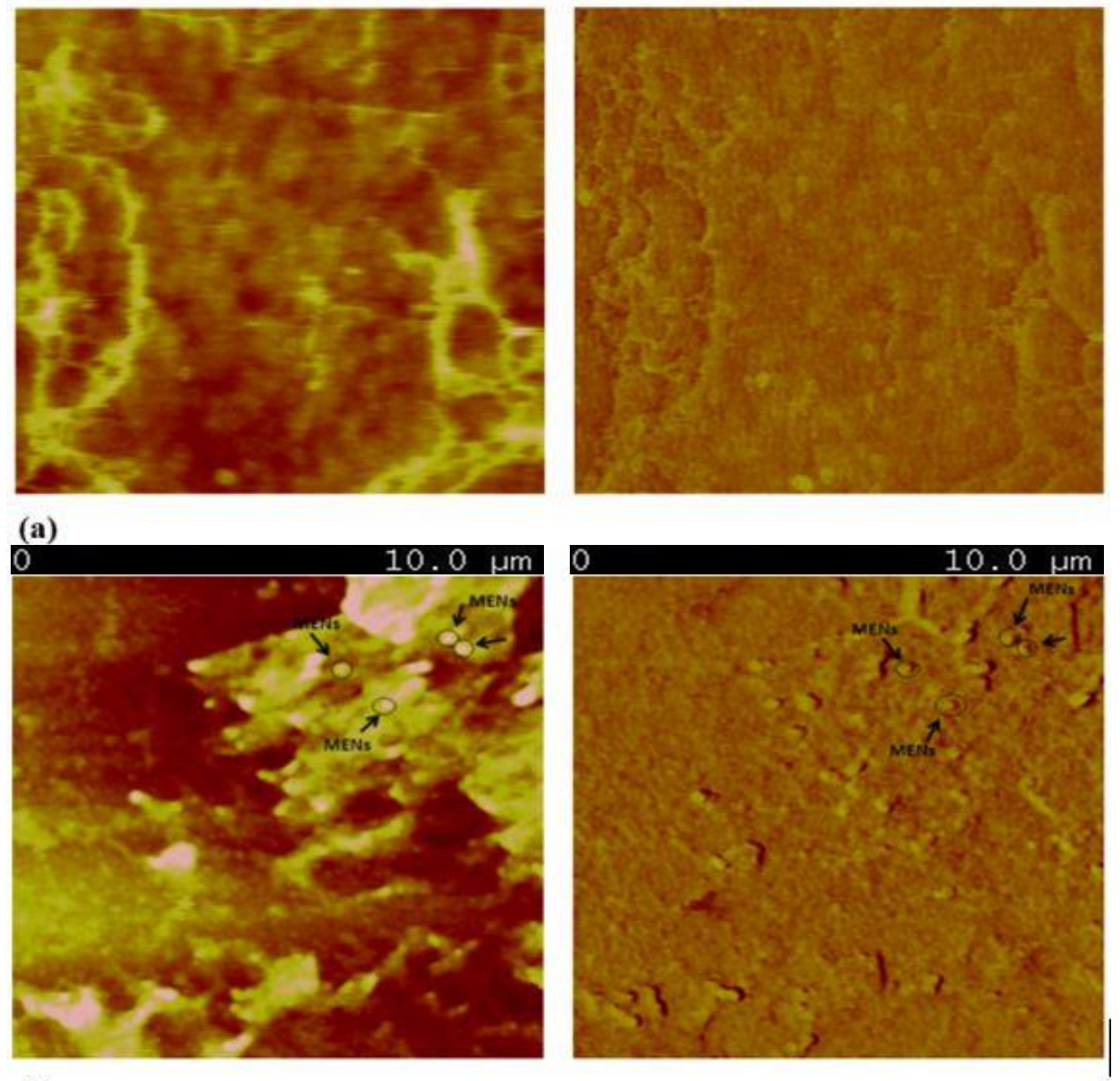

(b)

Figure 36: AFM image pair (z-height (left), phase (right)) of glioblastoma cells (a) without MENs, (b) with MENs

\subsection{Measurement of Cancer Cells}

In this study, MENs were integrated into media with different cancer cells and then their NMR spectrum was measured under equivalent conditions. 


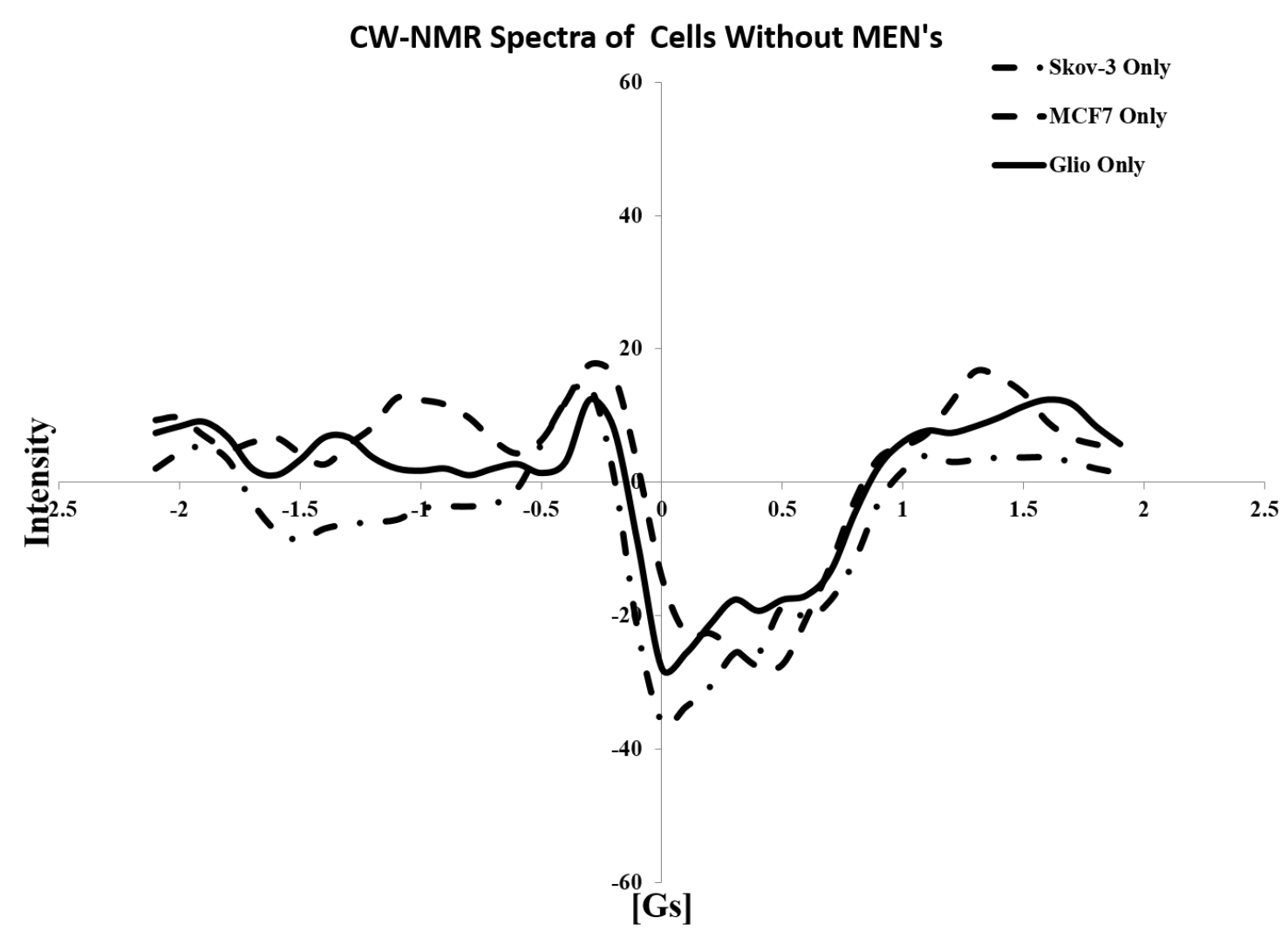

Figure 37: CW-NMR spectra of cells without MENs for ovarian carcinoma cells SKOV-3, breast adenocarcinoma cells MCF-7 and glioblastoma cells U87-MG.

Figure 37 shows continuous wave NMR curves for three cancer cell lines under study including glioblastoma cells, ovarian carcinoma cells SKOV-3, and breast adenocarcinoma cells MCF-7 without any nanoparticle being present in the media. The NMR signal is measured in the field sweep range from $-2.5 \mathrm{Gs}$ to $+2.5 \mathrm{Gs}$ and at a frequency of $14,000 \mathrm{kHz}$. It can be noted that the three cell lines don't significantly differ from each other. 

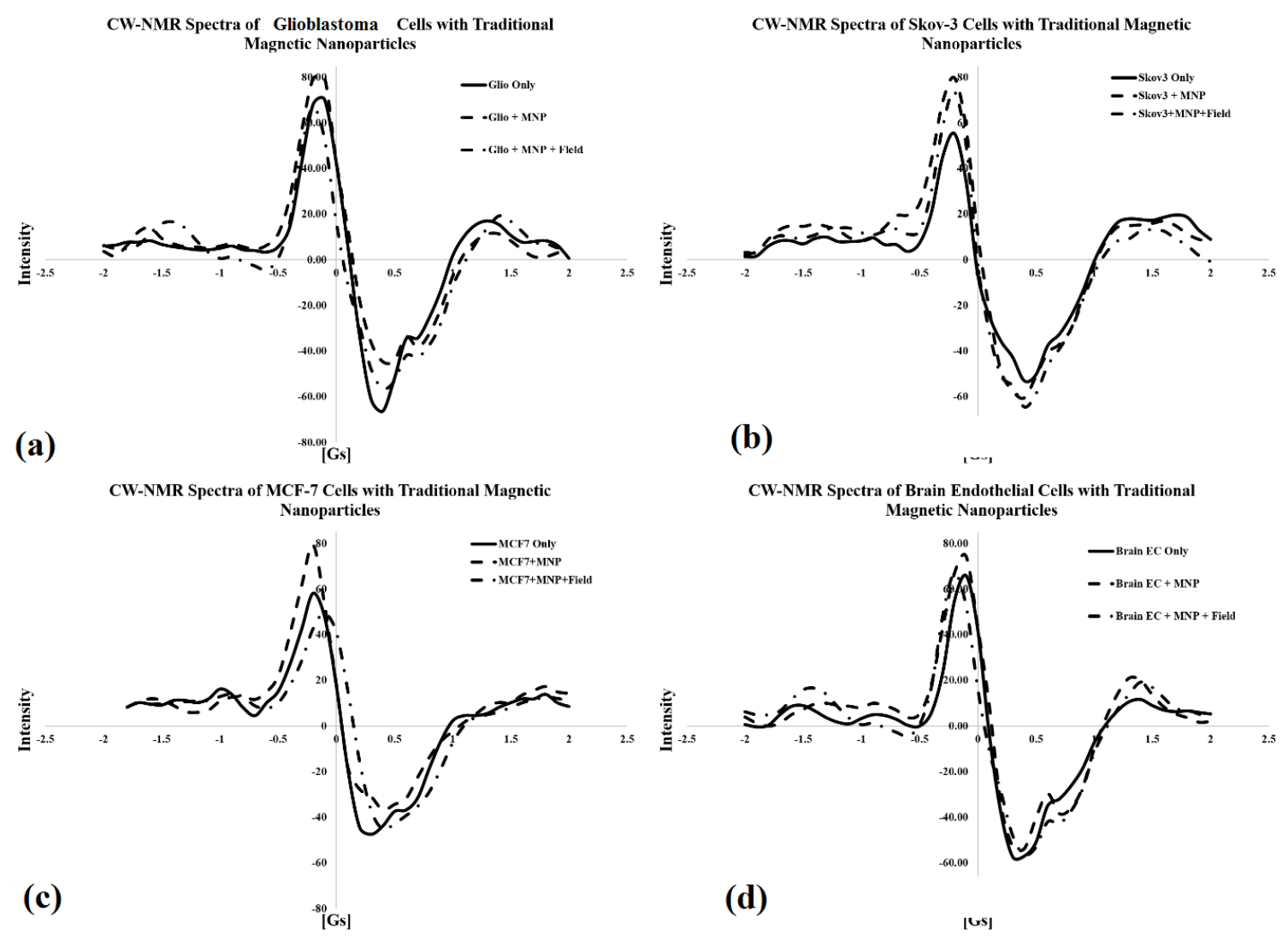

Figure 38: CW-NMR Spectra of (a) U87-MG, (b) SKOV-3, (c) MCF-7, and (d) Brain
Endothelial Cells with traditional magnetic nanoparticles

Figure 38 shows continuous wave NMR curves obtained by incubating three cancer cell lines (a) Glioblastoma, (b) SKOV-3, (c) MCF-7 and a non-cancerous healthy cell line (d) for 15 hours with traditional magnetic nanoparticles. Each set consists of three curves including spectra for cells only, cells incubated with MNPs without application of an external field, and cells incubated with MNPs under application of a 100-Oe d.c. field. The concentration of the nanoparticles in the media was approximately $150 \mu \mathrm{g} / \mathrm{ml}$. Similar to the case without any nanoparticles, neither of the spectra i.e. cells only (solid line), cells with magnetic nanoparticles (broken lines) and cells with magnetic nanoparticles with 
external magnetic field (dotted broken lines) for the four cell lines significantly differs from the corresponding cell line without MNs incubation as well as between each other.

However, the above trend drastically changes if MENs are used instead of MNPs.
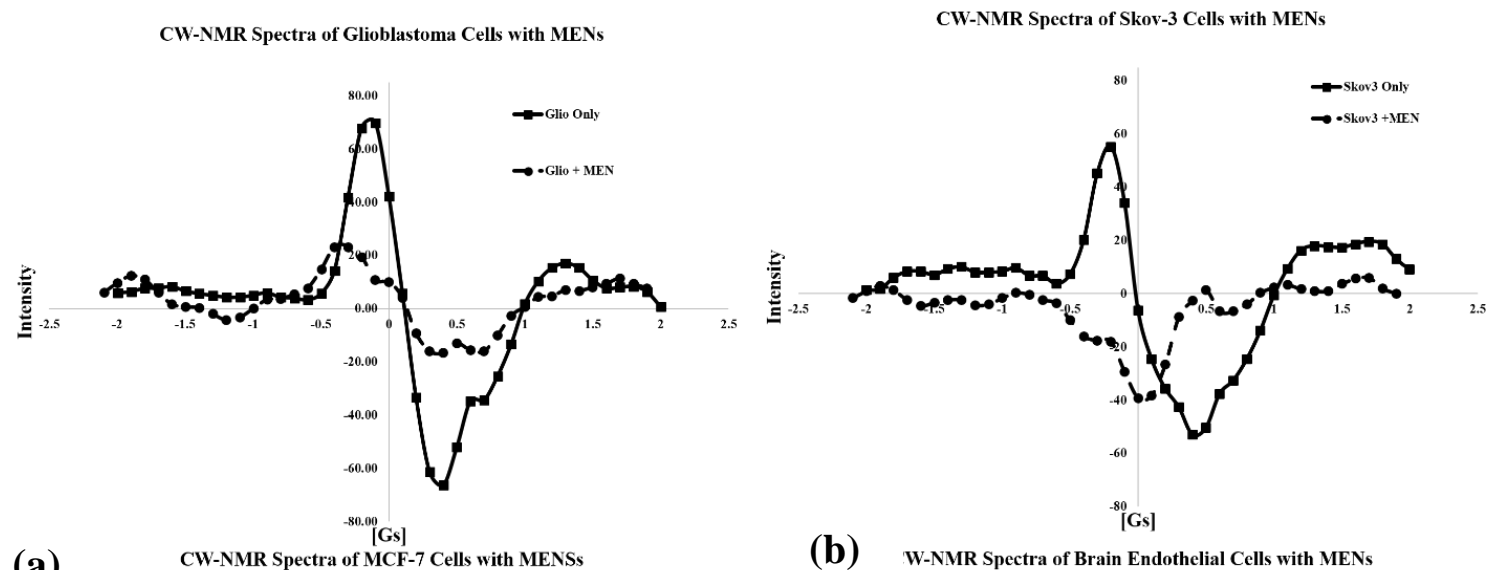

(a) CW-NMR Spectra of MCF-7 Cells with MENSs

(b) W-NMR Spectra of Brain Endothelial Cells with MENs
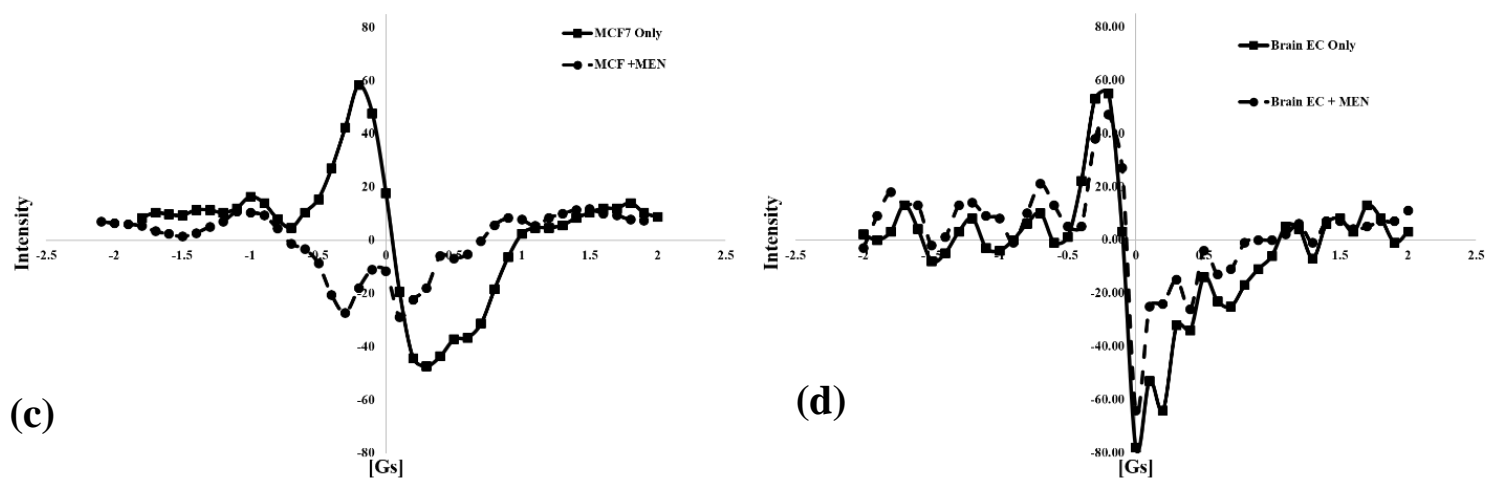

Figure $39 \mathrm{CW}-\mathrm{NMR}$ spectra of cells incubated with MENs for (a) glioblastoma cells U87MG, (b) ovarian carcinoma cells SKOV-3, (c) breast adenocarcinoma cells MCF-7, and (d) normal brain endothelial cells

Figure 39 shows CW-NMR spectra obtained by incubating the same three cancer cell lines and non-cancerous normal cell line for the same amount of time of 15 hours, with the only exception of having MENs instead of MNPs at the same concentration of approximately $150 \mu \mathrm{g} / \mathrm{ml}$. According to these spectra, in great contrast to the traditional 
MNPs, MENs significantly affects a NMR spectrum for each cancer cell type. The only exception is the non-cancerous endothelial cell line; as MNPs, MENs barely affected the spectrum.

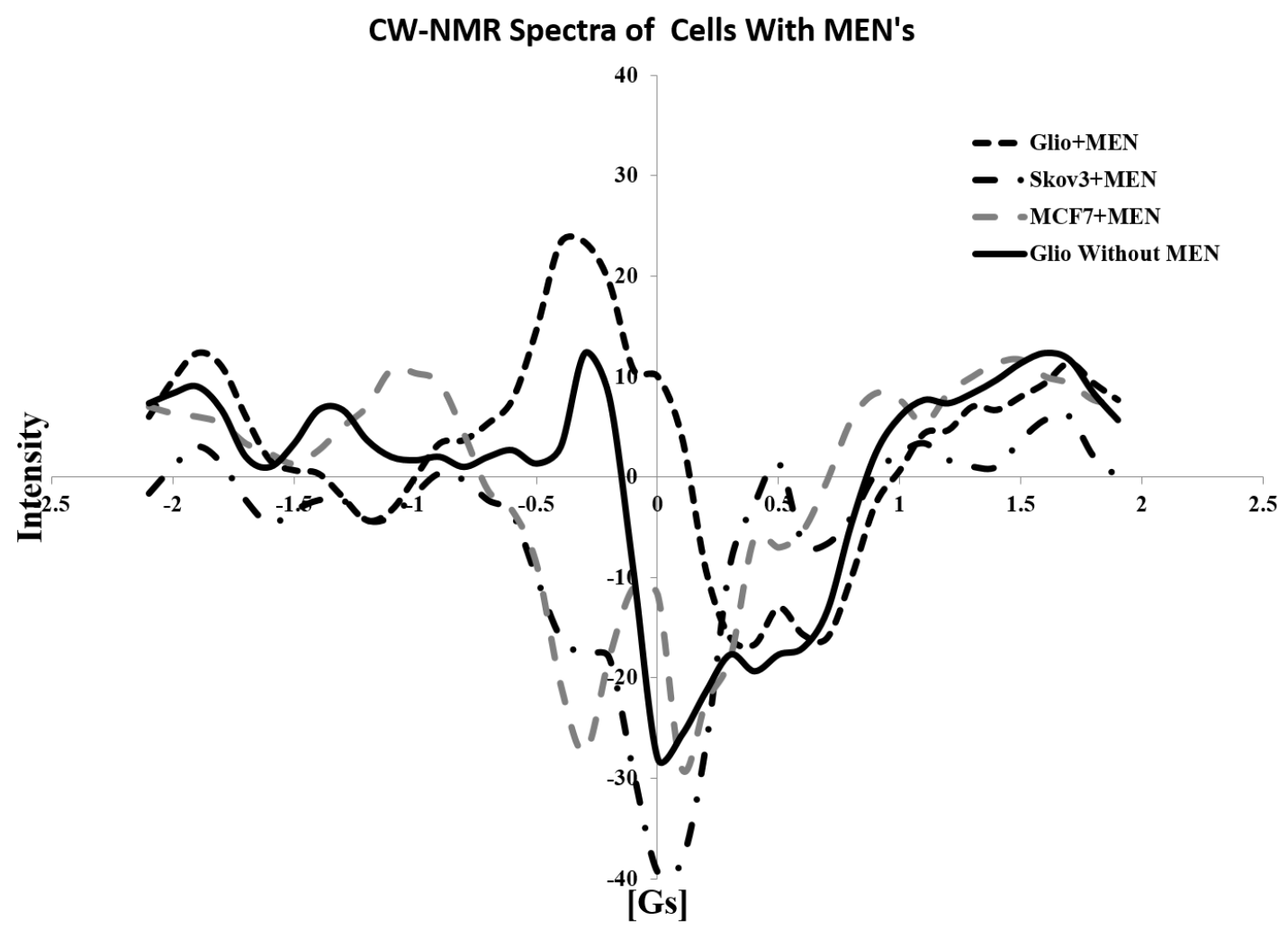

Figure 40: CW-NMR spectra of Glioblastoma, SKOV-3 and MCF-7 cells (broken lines as shown in the legend) with MENs and Glioblastoma cells without MENs (solid line)

Figure 40 shows continuous wave NMR curves for the same three cancer cell lines incubated with MENs without field application under equivalent conditions. The concentration of the nanoparticles was approximately $150 \mu \mathrm{g} / \mathrm{ml}$. Unlike the above case with the traditional MNs, the three cell lines Glioblastoma, SKOV-3 and MCF-7 significantly differ from each other. It can be noted that the difference between the spectra 
is not just quantitative but rather qualitative. Each cell type displays a distinguished set of peaks in its spectrum thus indicating an intrinsic interaction between MENs and cells. The spectra of Glioblastoma cells without any MENs (dark solid line) is provided for reference. 


\section{SIGNATURE ANALYSIS OF PROTHROMBIN}

\subsection{Background}

As we described in chapter 2, it is imperative to understand the functionality of proteins in our body that plays a vital role for disease prevention and cure. There are many applications of nanotechnology on computer engineering, sensing, biology and medicines, pathogen detection etc. however not much is done specific to functionality of protein that cause diseases [2-4,21-25]. Most of the nanomedicine technology rely on drug delivery through blood vessels so the toxicity of nanoparticles has been discussed but not its effect on the blood coagulation system [26-29]. Heart disease is the leading causes of death in the world. Ischaemic heart disease, stroke, lower respiratory infections and chronic obstructive lung disease have remained the top major killer diseases in recent years. It is critically important to control and monitor the Prothrombin Time (PT) in order to prevent from possible stroke or clot formation in blood vessels. We attempt to get the signature of prothrombin and its functionality using magnetoelectric nanoparticles.

\subsection{Prothrombin time and international normalized ratio}

Prothrombin time (PT) is a blood test that measures the time it takes for the plasma of your blood to clot. The measurement of prothrombin time depends on the reagent used in the laboratory. Unlike the standard laboratory test, viscoelastic method (VEM) is based on the whole blood coagulation available in real time at much faster turnaround time [68]. The prothrombin time is functional determination of the extrinsic (tissue factor) pathway of coagulation and is sensitive to the vitamin-K dependent clotting factors (factors II, VII, and $\mathrm{X}$ ). Tissue factor (factor III) is a transmembrane protein that is widely expressed on 
cells of non-vascular origin, which activates factor VII during the initiation of the extrinsic coagulation pathway.

Since the prothrombin time varies according to the type of analytical system employed and the reagent used to perform the test, World Health Organization (WHO) introduced a standard way called International Normalized Ratio (INR) [69]. Each manufacturer assigns an International Sensitivity Index (ISI) for any tissue factor they manufacture as a reagent. The ISI value indicates how a particular batch of tissue factor compares to an international reference tissue factor. The INR of a patient is given by the following equation.

$$
I N R=\left(\frac{P P T}{M N P T}\right)^{I S I}
$$

Where, PPT is the Patient Prothrombin Time for the given reagent (ISI), and MNPT is the Mean Normal Prothrombin Time.

The value of ISI is usually between 1 and 2. The INR of a healthy person is between 0.8 and 1.1 while the physicians recommend it to be between 2 and 3 for therapeutic patients.

Critically ill patients develop hemostatic abnormalities, ranging from isolated thrombocytopenia or prolonged global clotting tests to complex defects, such as disseminated intravascular coagulation. There are many causes of coagulation disorders depending the kind and condition of patients that require specific therapeutic or supportive management. In recent years, new insights into the pathogenesis and clinical management 
of many coagulation defects in critically ill patients have been accumulated and optimized the diagnostic and therapeutic strategy [70].

The use of certain anticoagulant therapy in patients with cases of blood related heart disease, stroke and pulmonary arterial hypertension (PAH) has been controversial for decades [71-74]. In this study, with the approved IRB and consent with the patient (the researcher), we are performing an experiment in human subject using blood sample of patient with Pulmonary Artery Hypertension WHO group I. The results of Comparative, Prospective Registry of Newly Initiated Therapies for Pulmonary Hypertension (COMPERA) lend support to current recommendations for the use of anticoagulant therapy in patients with idiopathic PAH, but not in other forms of PAH [7476]. But, it is inevitable that the proper use of anticoagulant lowers the risk of heart failure provided that there are no other side effects.

\subsection{Sample Preparation}

MENs was prepared as described in chapter 2.3 with slight modification. $\mathrm{CoFe}_{2} \mathrm{O}_{4}$ $\mathrm{BaTiO}_{3}$ core shell MENs were prepared according to the following conventional procedure. As the first step, $\mathrm{CoFe}_{2} \mathrm{O}_{4}$ particles were prepared by the standard hydrothermal method, according to which $0.058 \mathrm{~g}$ of $\mathrm{Co}\left(\mathrm{NO}_{3}\right)_{2} .6 \mathrm{H} 20$ and $0.16 \mathrm{~g}$ of $\mathrm{Fe}\left(\mathrm{NO}_{3}\right) 3.9 \mathrm{H} 20$ were dissolved in $15 \mathrm{ml}$ of distilled water and $0.2 \mathrm{~g}$ of polyvinylpyrrolidone was dissolved in 5 $\mathrm{ml}$ of aqueous solution containing $0.9 \mathrm{~g}$ of sodium borohydride at $120^{\circ} \mathrm{C}$ for 12 hours. Then, a precursor solution of $\mathrm{BaTiO}_{3}$ was prepared by mixing $30 \mathrm{ml}$ of aqueous solution containing $0.029 \mathrm{~g}$ of $\mathrm{BaCO}_{3}$ and $0.1 \mathrm{~g}$ of citric acid with $30 \mathrm{ml}$ of ethanolic solution containing $1 \mathrm{~g}$ of citric acid and $0.048 \mathrm{ml}$ of titanium (IV) isopropoxide. Coreshell 
$\mathrm{CoFe}_{2} \mathrm{O}_{4}-\mathrm{BaTiO}_{3} \mathrm{MENs}$ were prepared by mixing $0.1 \mathrm{~g}$ of $\mathrm{CoFe}_{2} \mathrm{O}_{4}$ nanoparticles in the $\mathrm{BaTiO}_{3}$ precursor solution and the mixture was sonicated for $2 \mathrm{hrs}$. Once the $\mathrm{CoFe}_{2} \mathrm{O}_{4}$ nanoparticles were thoroughly dispersed, the mixture was dried on the hot plate at $60^{\circ} \mathrm{C}$ overnight while being continuously stirred. The dried powder was heated to $780^{\circ} \mathrm{C}$ for 5 hrs. in a furnace (CMF-1100) and cooled at $52^{\circ} \mathrm{C}$ per minute to obtain the coreshell MENs of $\sim 30 \mathrm{~nm}$ diameter. The particles size distribution was measured using dynamic light scattering method (Malvern-Zetasizer).

Blood sample was taken via finger prick from a patient of Pulmonary Arterial Hypertension, WHO Group I. The blood sample was taken in a pipette and gently transferred into a micro centrifuge tube containing MENs. Details are in the following chapter.

\subsection{Measurement of therapeutic blood}

Blood consist of erythrocytes or red blood cells (RBC), leukocytes or white blood cells (WBC), and thrombocytes or platelets in plasma. It contains ferromagnetic materials especially in RBCs that is expected to interact with MENs. Most of the therapeutic drug used to control PAH is routed via blood so as the drug coated magnetoelectric nanoparticles (MENs). Interestingly, there is very little or none interaction of MENs on blood cells rather the experiment shows a significant effect on PT/ INR of the blood. Our objective is to analyze the interaction of MENs with the INR of therapeutic blood or other blood constituents especially the hemoglobin or hematocrit. With the approval of IRB protocol and consent from the Office of Research Integrity at FIU and all required trainings for 
Human Subject Research, therapeutic blood sample of a pulmonary arterial hypertension patient is taken for the study.

Sample of blood from a PAH patient, New York Heart Association (NYHA) Functional Class III, was collected via finger prick. The measured INR of the blood was 2.9, hemoglobin $18.9 \mathrm{gm} / \mathrm{dL}$ and hematocrit 58\%. After treating with MENs of concentration $50 \mathrm{mg} / \mathrm{mL}$, the INR increased to 4.1 however, the aftermath measurement of INR will not reflect the actual reading due to time exposed and concentration of the sample. The cougulometer may not function properly under such environment. It may indicate that the MENs has higher impact on the prothrombin time of blood. From the engineering prospective, experiments show a distinct signature of INR of therapeutic blood using MENs. In-depth analysis using molecular dynamics computational simulation will give the actual interaction mechanism of MENs on prothrombin. Currently, we are working to understand the underlying physics behind it. We tried to measure the INR with the lower concentration of MENs but failed to get the readings from cougulometer. A measured amount of citrate was added as a reversible anticoagulant to study its interaction with the nanoparticles using NMR.

$25 \mu \mathrm{L}$ of MENs Concentration $50 \mathrm{mg} / \mathrm{mL}$ was taken in a micro centrifuge tube with equal amount of therapeutic blood sample. Out of the sample, only one capillary was taken for NMR measurement. The CW-NMR spectrometer results are shown in the following graphs along with their signature curves. 


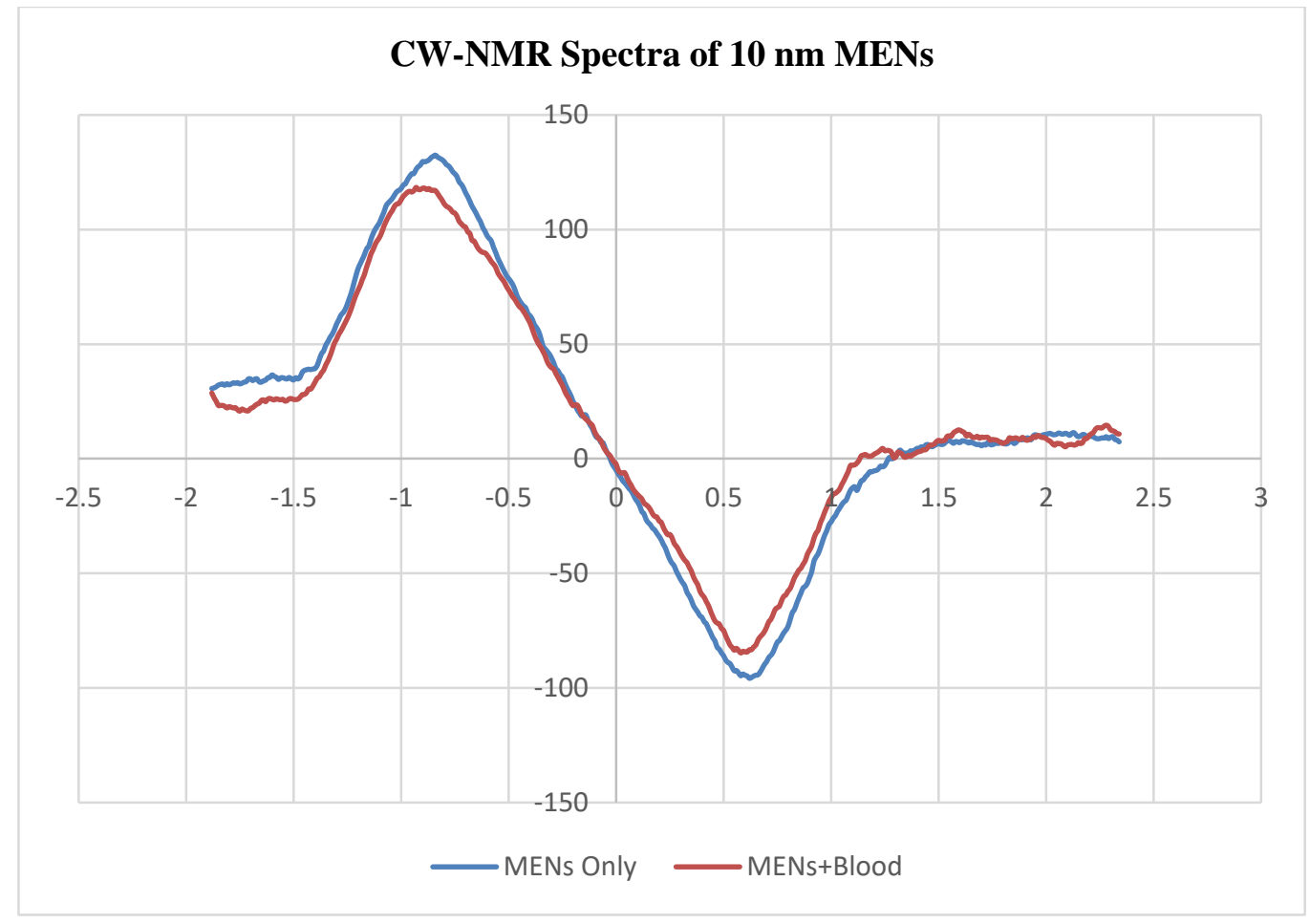

Figure 40: Comparison of $10 \mathrm{~nm}$ MENs background (blue) and MENs with blood sample (red)

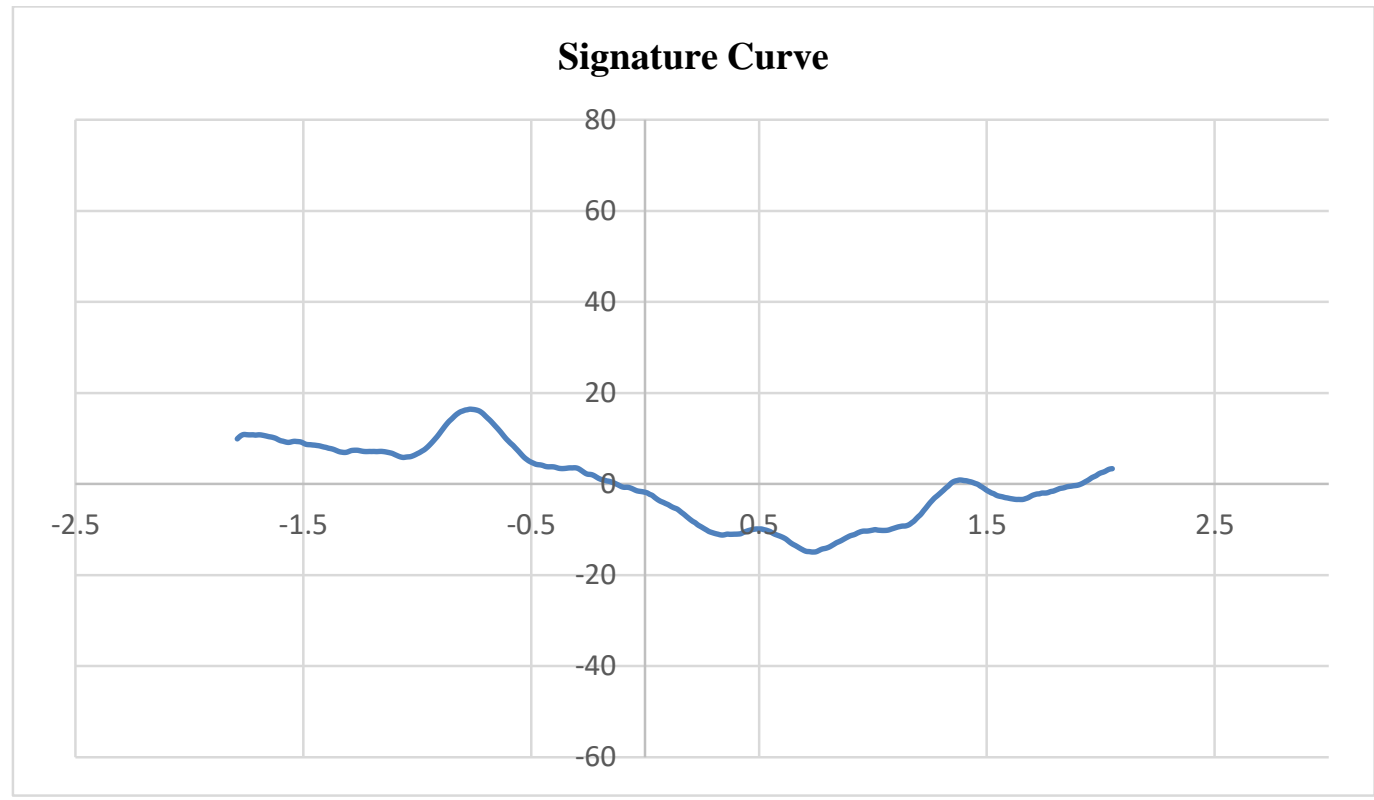

Figure 41: Signature curve of $10 \mathrm{~nm}$ MENs with sample blood 


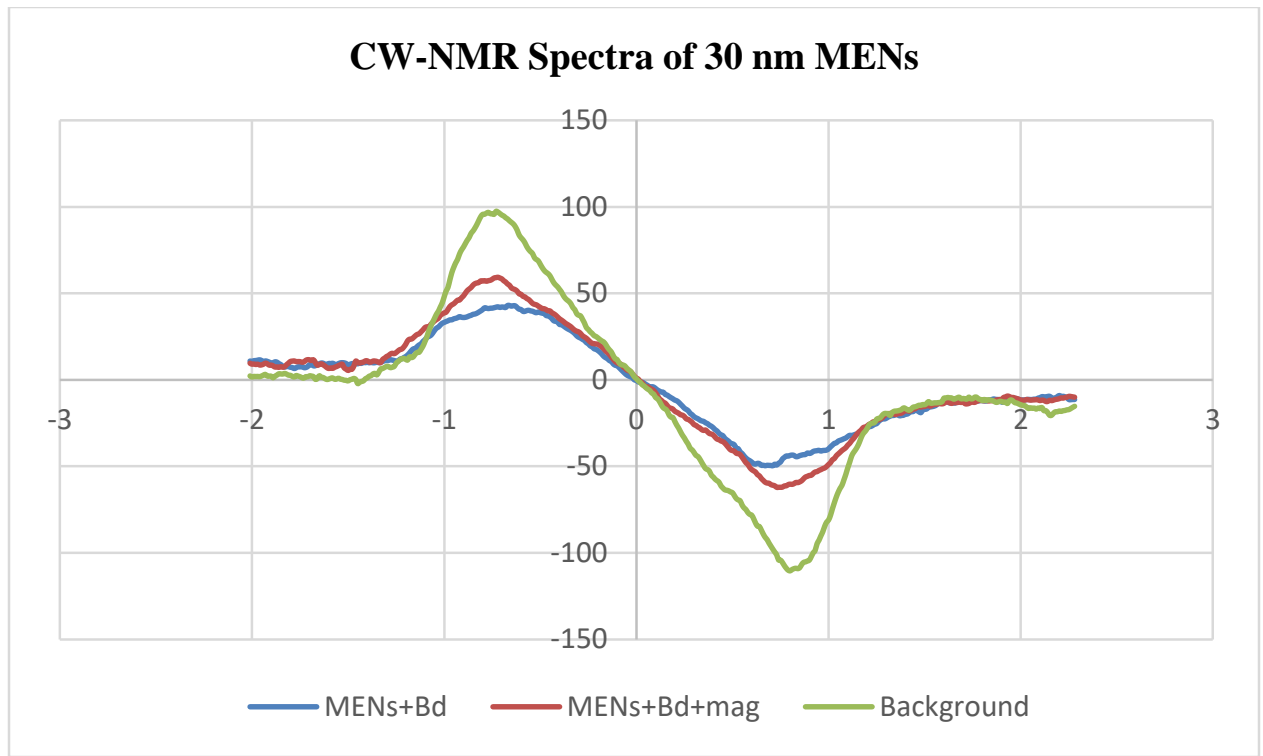

Figure 42: Comparison of $30 \mathrm{~nm}$ MENs background (green), MENs with blood sample (blue) and MENs and blood sample with magnetization (red)

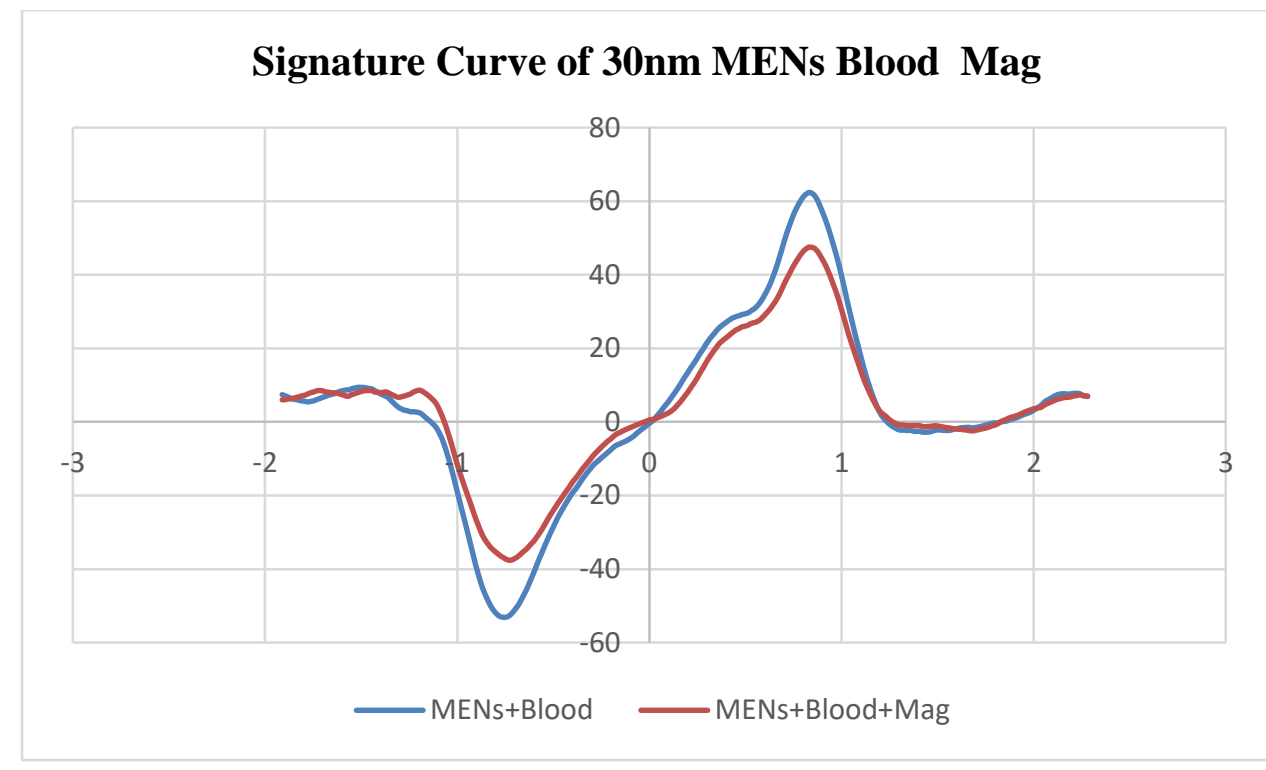

Figure 43: Signature curve of $30 \mathrm{~nm}$ MENs with sample blood (blue) and MENs and sample blood with magnetization (red)

Temporary magnetization of MENs (red) does effect the surface interaction with blood constituents but not significantly. 


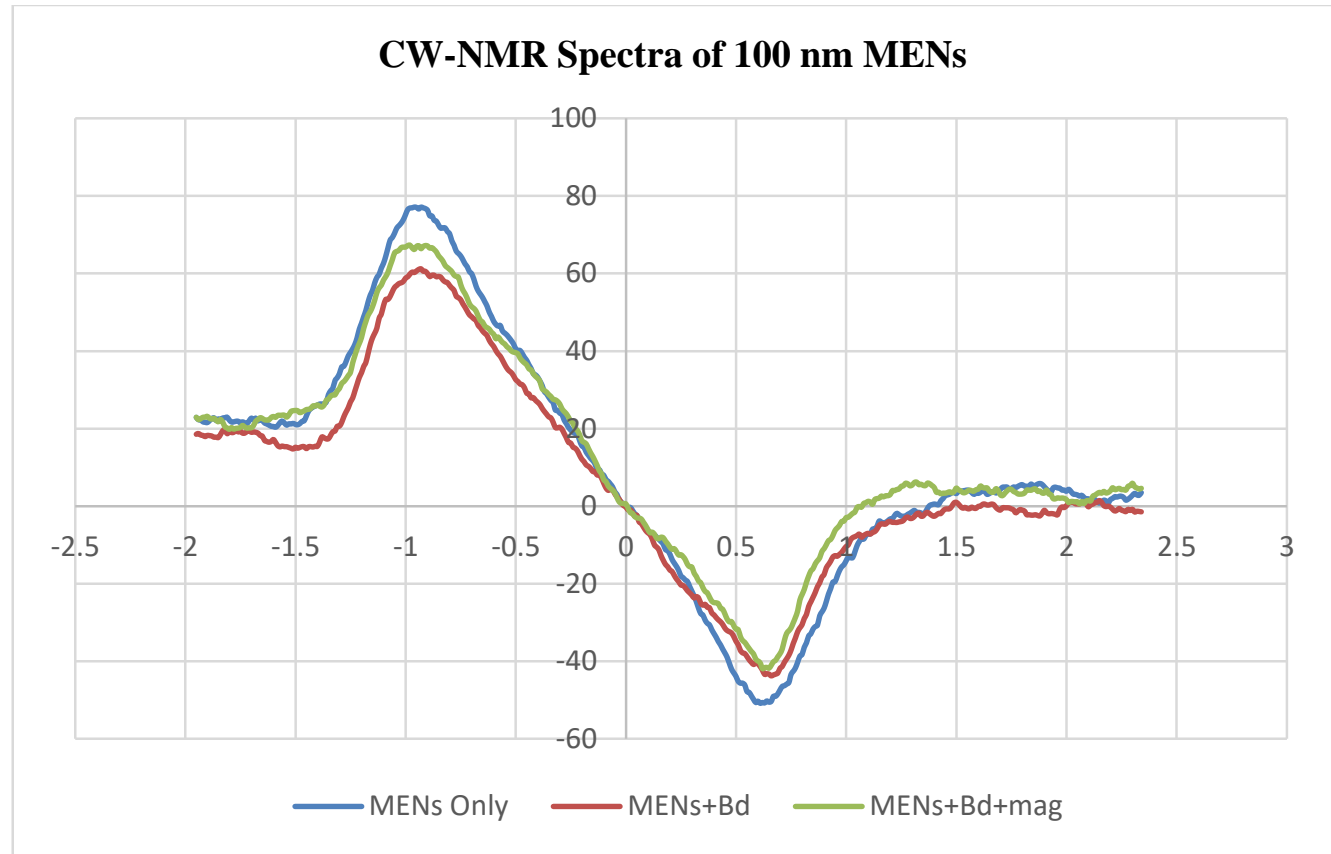

Figure 44: Comparison of $100 \mathrm{~nm}$ MENs background (blue), MENs with blood sample (red) and MENS and blood sample with magnetization (green)

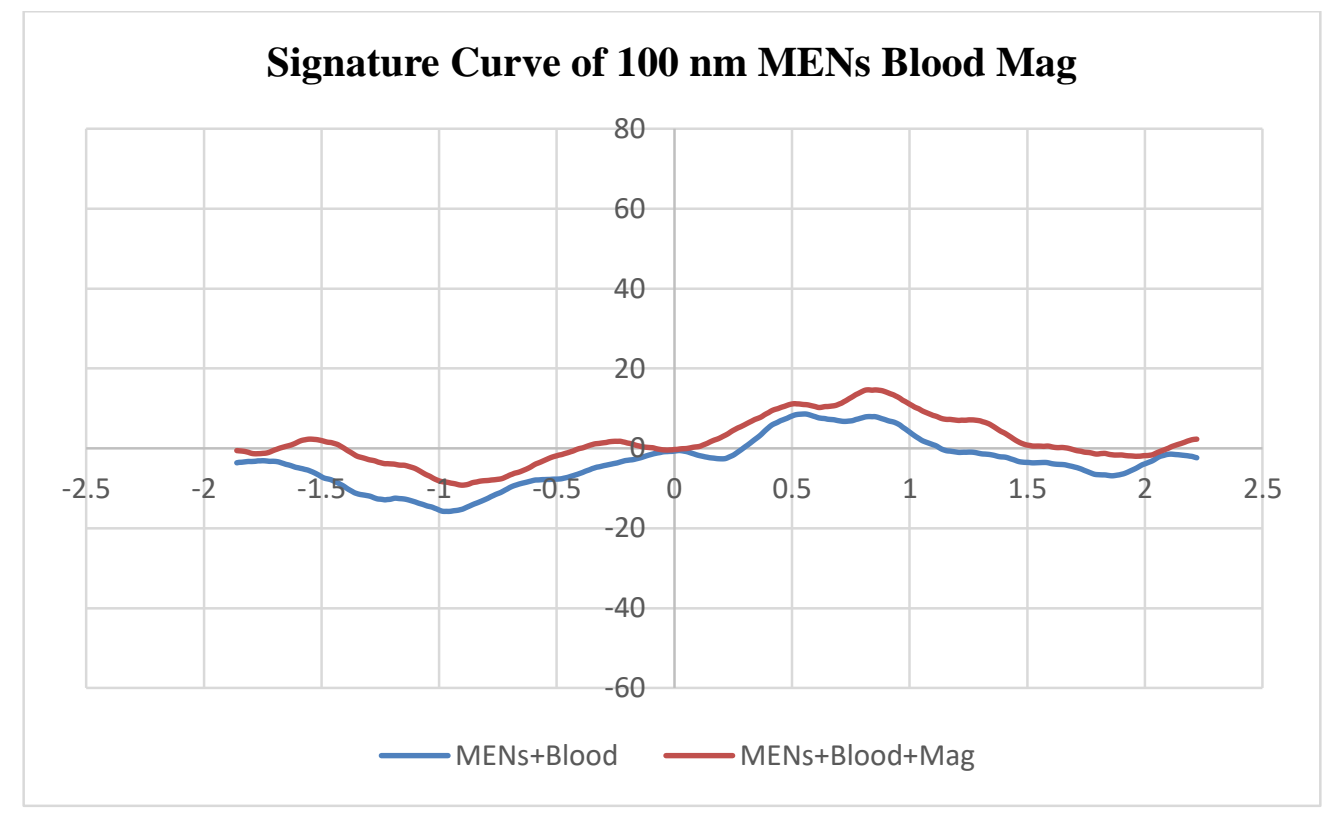

Figure 45: Signature curve of $100 \mathrm{~nm}$ MENs with sample blood (blue) and MENs and sample blood with magnetization (red) 
As usual, $\mathrm{X}$-axis in Gs and Y-axis is in arbitrary units. Figure 42 shows the efficacy of $30 \mathrm{~nm}$ MENs is greater than $10 \mathrm{~nm}$ (Figure 40) and $100 \mathrm{~nm}$ (Figure 44). Moreover, temporary magnetization does not have significant effect on the signature curve.

For further study we chose 30nm MENs for the experiment due to its higher efficacy. The blood sample of therapeutic patient was taken by finger prick as in the previous experiment with measured INR 1.4. Next, $500 \mu \mathrm{L}$ of blood sample with the concentration of $500 \mu \mathrm{g} / \mathrm{mL}$ of MENs was taken in a NMR tube for measurement. Figure 46 shows the CW-NMR spectra of the blood sample (INR 1.4) with MENs. Sample placement, instrument parameter like $\mathrm{B}_{0}$, phase, and line width were carefully selected to ensure optimal signal to noise ratio. NMR spectra was collected at opposite phases $107^{\circ}$ (blood with MENs) and $297^{\circ}$ (background). The spectra from blood sample and MENs background was added with opposite phase signal to eliminate the possible solvent interference. Field sweep was taken as $50 \mathrm{Gs}$ with the frequency of $14,000 \mathrm{kHz}$.

The INR of therapeutic patients varies depending on the physical activity, diet and various other factors so INR should be monitored such that it does not go down to around 1 or up to around 4 . The patient has been monitored with prescribed warfarin from $5 \mathrm{mg}$ to $7.5 \mathrm{mg}$ per day since last several years. During regular checkup, at a point when the measured INR was 1.4, we took the blood sample and used for CW-NMR spectra. It is hard to keep diet (especially that interferes with vitamin K), exercise, supplemental oxygen, and other physical activities as normal for such an active patient so it is evident that the INR varies from time to time, therefore it needs to be monitored every 2 to 4 weeks and change the dose of warfarin accordingly. At some other time when the INR was 3.1, the blood 
sample was taken again for measurement. Same as before, $500 \mu \mathrm{L}$ of blood sample with the concentration of $500 \mu \mathrm{g} / \mathrm{mL}$ of MENs was taken in a NMR tube for measurement. Figure 47 shows the CW-NMR spectra of the blood sample (INR 3.1) with MENs.

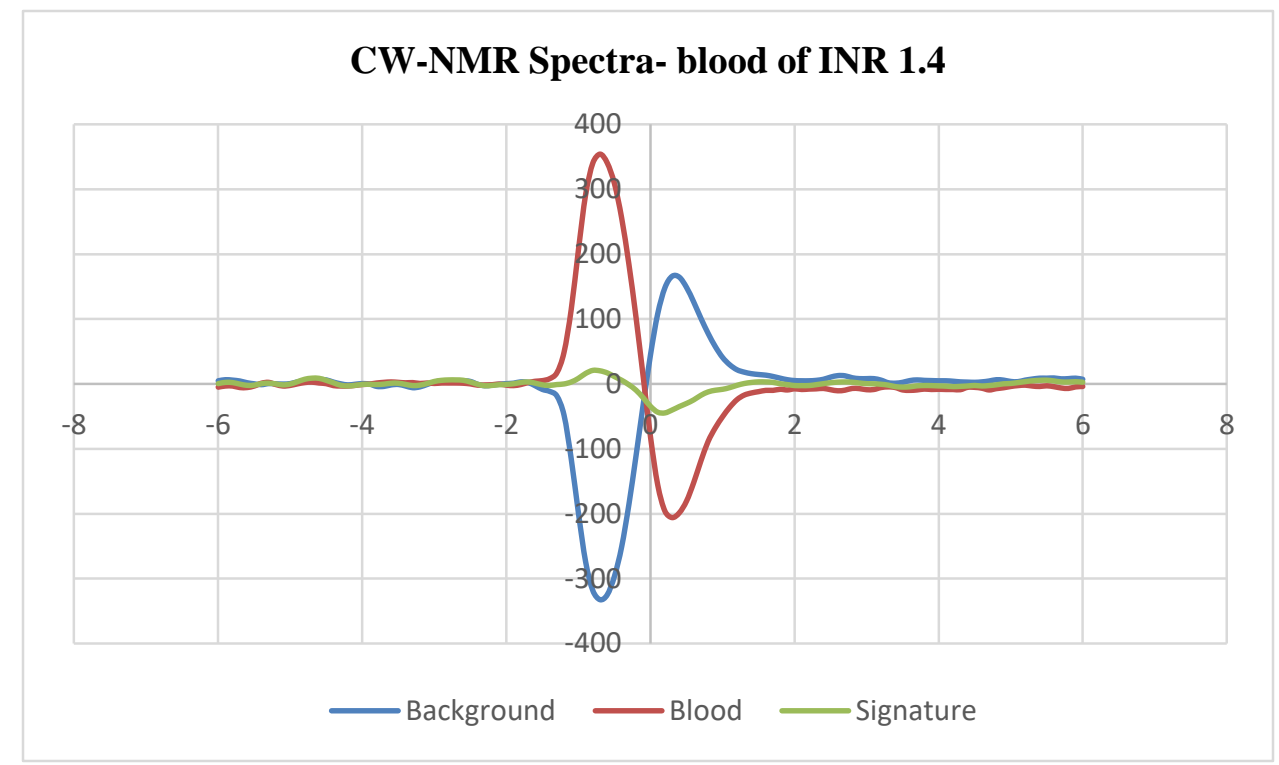

Figure 46: CW NMR Spectra of MENs with therapeutic blood of measured INR 1.4 


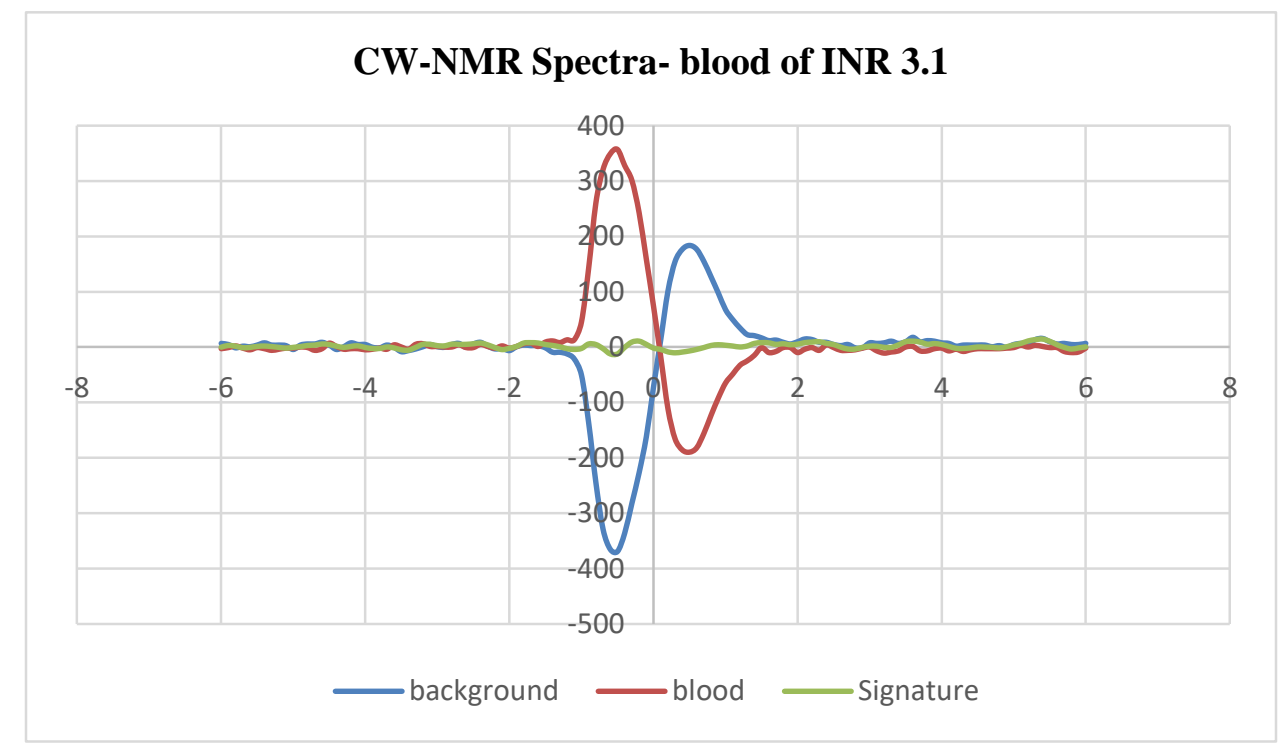

Figure 47: CW NMR Spectra of MENs with therapeutic blood of measured INR 3.1

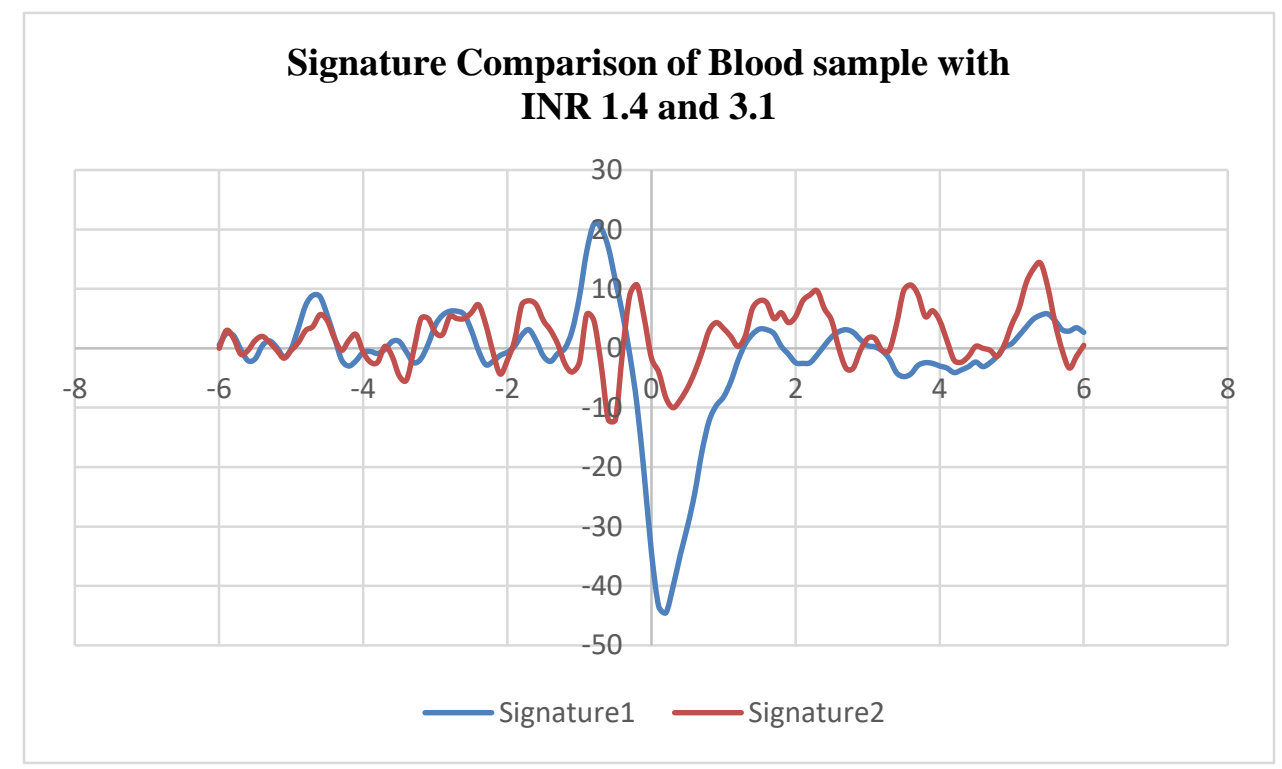

Figure 48: CW-NMR Signature comparison of blood samples with measured INR 1.4 (blue) and 3.1 (red)

Figure 48 shows that the signature of blood with lower INR (1.4) has greater intensity compare to the higher INR (3.1). Higher INR means thin blood where only few linkers 
connecting kringles of prothrombin are active. Lower INR means dense blood that has high efficacy with the MENs so that we can see a significant peak in the graph. We are in the process of performing high resolution computational molecular dynamics simulation which may reveal the in-depth understanding of molecular mechanism of the system.

\subsection{Loess Method}

Most of the times we used Loess Method for the graph plot. There are several methods of smoothing the curves where moving averages is widely used by engineers when the variables are somewhat linearly distributed. We attempt to use an alternate way, mostly used by economists, called Loess method that strongly relates to non-parametric regression methods that combine multiple regression models in a k-nearest-neighbor-based meta-model. It can also be referred as LOcal regrESSion. In the loess method, weighted least squares are used to fit linear or quadratic functions of the predictors at the centers of neighborhoods. The radius of each neighborhood is chosen so that the neighborhood contains a specified percentage of the data points. The fraction of the data, called the smoothing parameter, in each local neighborhood controls the smoothness of the estimated surface. Data points in a given local neighborhood are weighted by a smooth decreasing function of their distance from the center of the neighborhood. SAS Analysis with the LOESS Procedure and dependent variable: yax is the intensity in arbitrary units. 


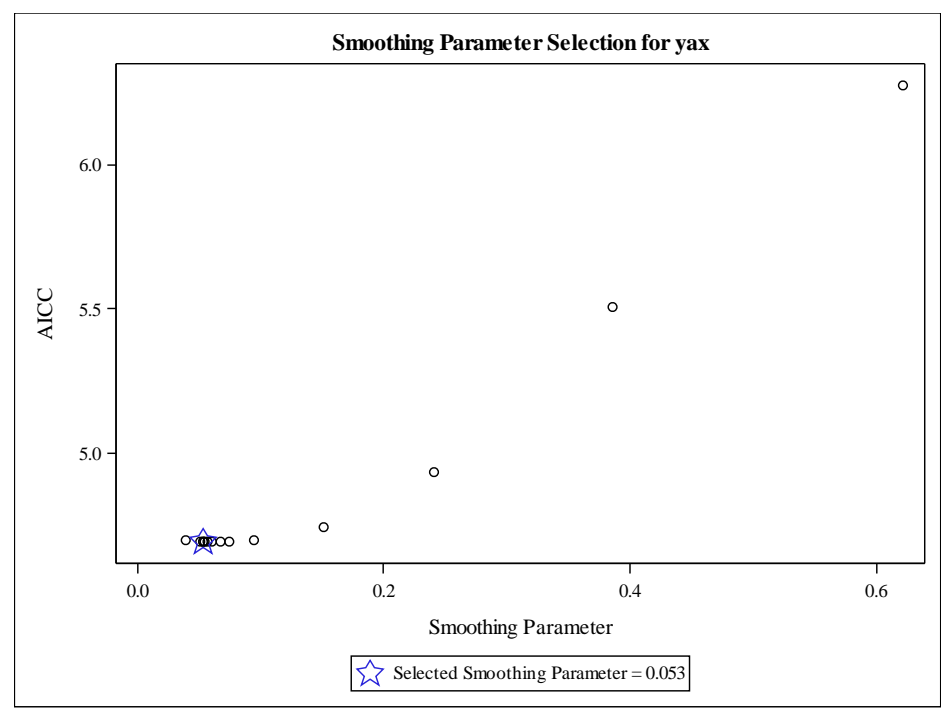

Figure 49: Selection of smoothing parameter

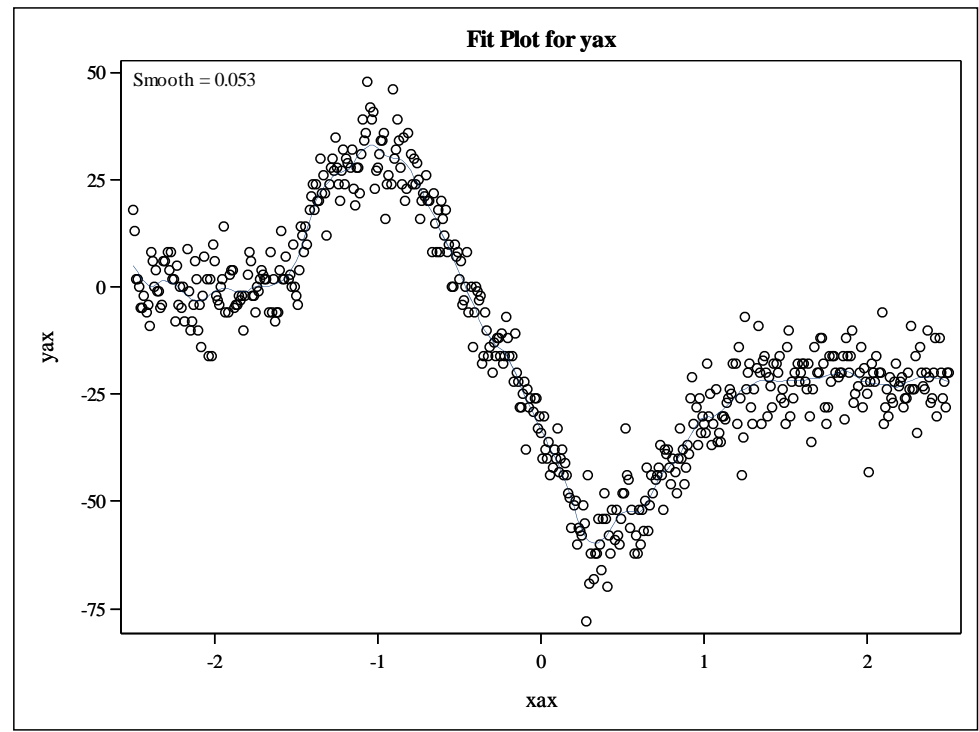

Figure 50: Figure 5.5: Curve fitting with smoothing parameter 0.053

Smoothing parameter was found to be 0.053 from Figure 44 . Figure 45 shows the best fit curve along with all the data points. 
There is no significant interaction of blood constituents with magnetoelectric nanoparticles and is save to use in medical applications however, the PT- INR shows a significant increment which could in other way be beneficial for the patients in terms of anticoagulation therapy.

\subsection{Sample blood with MENs coated with GMO and EDC}

Another sample of blood from the same subject is collected via finger prick. The measured INR of the blood was 2.8, hemoglobin $19.0 \mathrm{gm} / \mathrm{dL}$ and hematocrit $58 \%$. After treating with MENs of $2.5 \mathrm{mg}$, the Coagulation Meter showed the INR of 5.0. We must take into account that the time exposed to the blood after treating with MENs and the concentration of the blood have significant effect on INR and the coagulation meter will not reflect the actual value of PT or INR. The sample were treated MENs with GMO (GMO coated MENs sample preparation is discussed in Chapter 2.3.3) and with EDC separately. $20 \mathrm{uL}$ of MENs Concentration $50 \mathrm{mg} / \mathrm{ml}$ was taken in a micro centrifuge tube with $30 \mathrm{uL}$ of therapeutic blood sample.

Since this study is only to find the interaction of two intermediate layers GMO and EDC, and the CW-NMR spectra is significantly different, here we have shown the direct plot without using Loess Method for signal smoothing. 


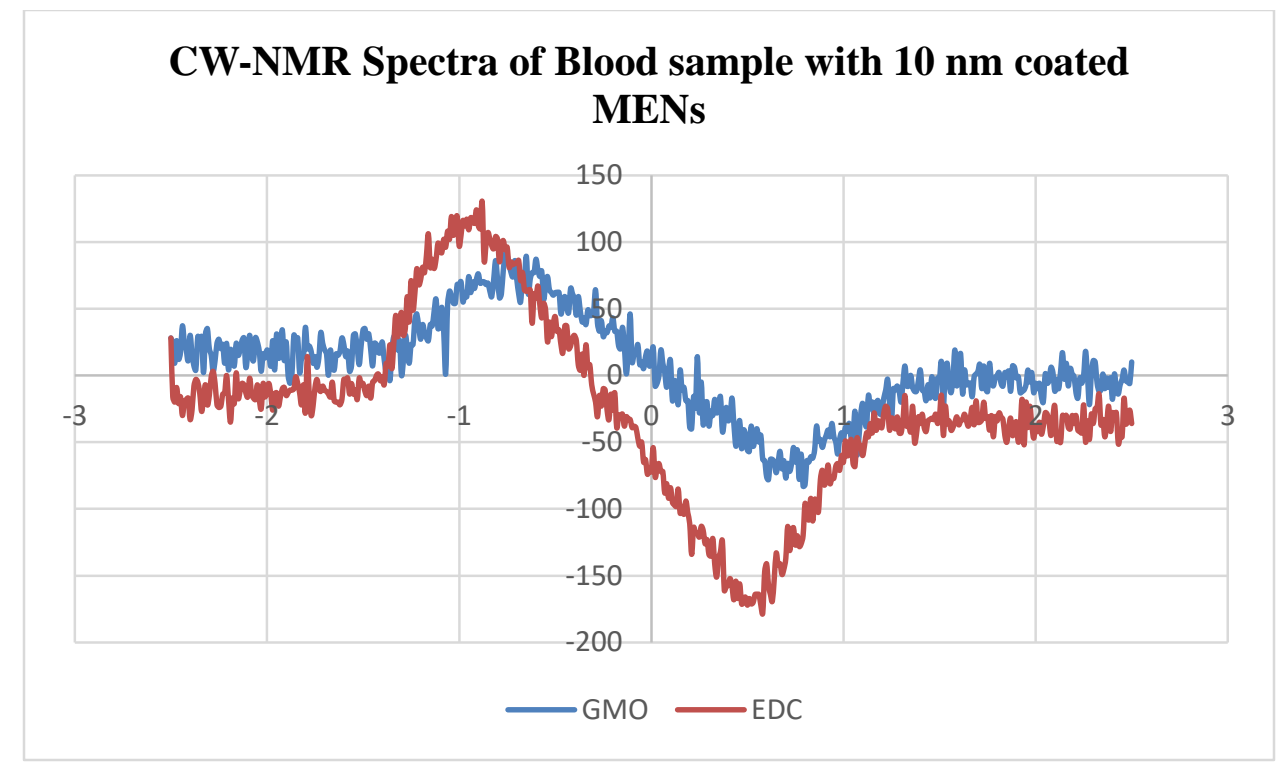

Figure 51: Comparison of GMO and EDC coated $10 \mathrm{~nm}$ MENs with blood sample

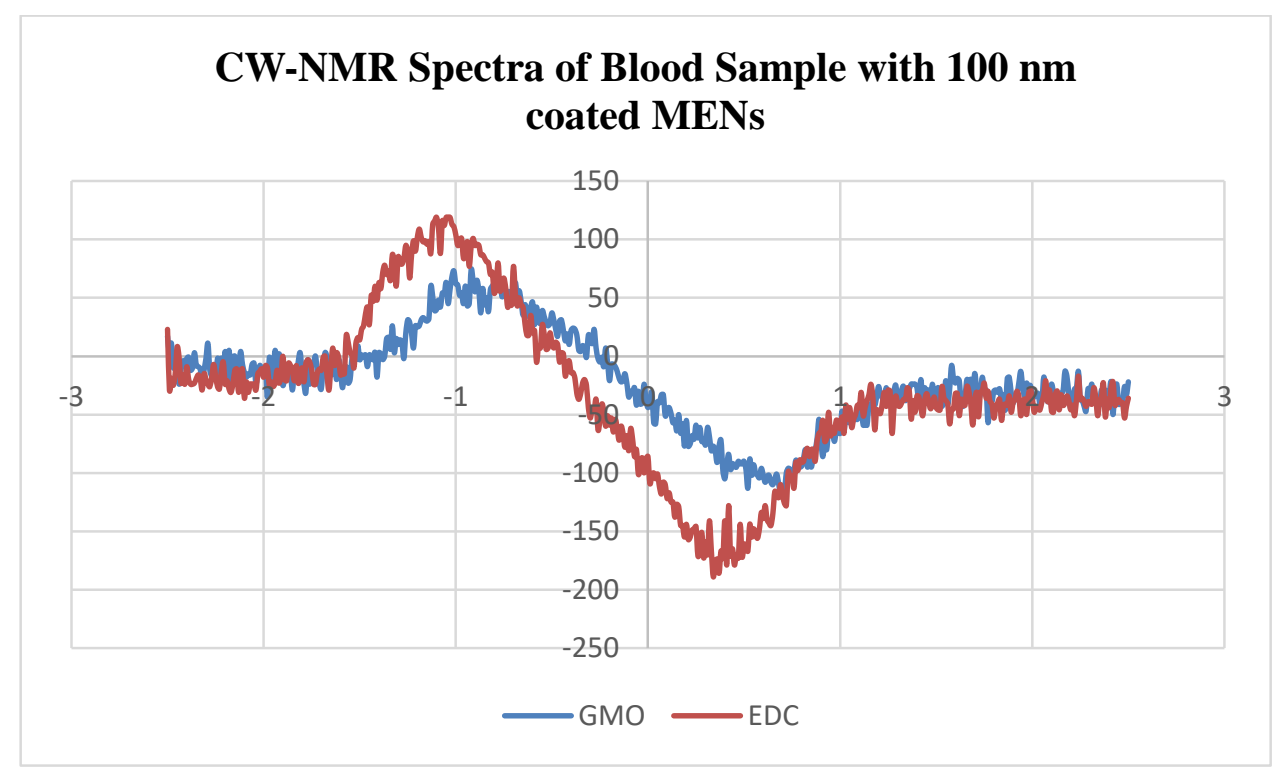

Figure 52: Comparison of GMO and EDC coated $100 \mathrm{~nm}$ MENs with blood sample 
It is clear that the MENs can also acts as a contrasting agent and safe and non-toxic to human blood however, the computational Physics, molecular dynamics study will explain what triggers the molecular mechanism of blood constituents that results in increment of PT/ INR. The graphs Figure 51 and Figure 52 are CW-NMR spectra of GMO and EDC coated MENs of size $10 \mathrm{~nm}$ and $100 \mathrm{~nm}$ respectively. EDC coated MENs can better function for the signature of not only therapeutic blood sample but also with other biomolecular organisms. Currently, we are working with the Theoretical BioPhysics Group at the department of Physics at FIU to find out the underlying mechanism of molecular dynamics of prothrombin and nanoparticles interaction. 


\section{DISCUSSION}

The above experiments have clearly demonstrated that MENs are uniquely positioned to distinguish different cells and mircoorganisms from each other through identification of the samples' electric-field properties at the subcellular level. In this study, MENs have been compared to traditional magnetic nanoparticles which don't display any magnetoelectric effect and thus, unlike MENs, don't produce any significant magneticfield-dependent electric field of their own. In other words, the experiments have proven that it is the presence of the unique ME effect which sets MENs apart from all the other nanoparticle types and turn them into a superior diagnostic tool. To demonstrate the fundamental nature of this diagnostic approach, three independent applications have been studied including identification of blood cells, bacteria, and cancer cells. All three types of aforementioned biomolecular organisms can have vital impacts on the modern state of disease prevention and life quality. To present the underlying story, this discussion will focus on one particular application: cancer cells. For example, in Chapter 5, the significance of the cancer related application cannot be underestimated. Again, only MENs, not traditional purely magnetic nanoparticles, could detect the fine NMR spectral features which originate from complex intrinsic charge configuration on the membrane surface of a specific cell type.

The following back-of-the-envelope model could give an idea of the underlying physics of the diagnostic of cancer cells with MENs. When nanoparticles are placed in any cellular media, they bind to characteristic sites of the surface of cellular membrane such that the electric and chemical energy are minimized. Indeed, this could be seen in AFM images which clearly showed the nanoparticles "stick" to ruptured regions on the 
glioblastoma cellular membrane surface. In contrast, the normal endothelial brain cell membranes displayed a relatively continuous rupture-free surface with no preferential binding sites. In case of a MEN, depending on the binding site, the intrinsic electric field is affected in a certain way due to the electrostatic and chemical bonds at this site. Because, unlike the traditional MNs, MENs have a non-zero electric dipole, the electrostatic bonds can be particularly strong. Previously, MENs were shown to acquire a non-zero surface charge due to the double-layer chemistry in the cellular microenvironment. Due to the ME effect the resulting change in the electric field would trigger a change in the magnetic moment of the nanoparticle, which in turn would induce a change of the local magnetic field at this particular location. Considering there are many nanoparticles, they would preferentially bind to all the characteristic sites and thus would change the net magnetic field in a very specific way depending on the cell type. Thus, the effect could be quantified according to the following formulation.

At each binding site, the NMR signal, e.g. the electromagnetic energy absorption, is generated when the following resonance condition is satisfied: $h \omega=M_{n} H_{n e t}$, where $h$ is the Plank constant, $\omega$ is the frequency of the electromagnetic wave, $M_{n}$ is the magnetic moment due to the nuclear spin (in this study, the proton spin), $H_{n e t}$ is the net magnetic field at this location. This local net field is made of the two contributions, the external field generated by the NMR magnets, $H_{0}$, and the field due to the ME effect of MENs at the location, $H_{M E}$, respectively:

$$
H_{\text {net }}=H_{0}+H_{M E}
$$


To a zeroth approximation, according to the ME dependence, in the vicinity of a nanoparticle, $H_{M E} \sim M_{S}=\alpha E$, where $M_{S}$ is the nanoparticle's saturation magnetization, $\alpha$ is the ME coefficient, and $E$ is the electric field in the vicinity of the nanoparticle. In turn, this electric field depends on the cell membrane morphology and the interaction between the binding site and the nanoparticle. Assuming an electric field, $E$, on the order of 1000 $\mathrm{V} / \mathrm{cm}$, a ME coefficient, $\alpha$, of $10 \mathrm{mVcm}^{-1} \mathrm{Oe}^{-1}$, according to the ME expression, the induced local magnetic field, $H_{M E}$, could be on the order of $10 \mathrm{Oe}$, which would make a significant contribution to the NMR signal. The NMR bias field, $H_{0}$, is on the order of 2,000 Oe. Here, it should be noted that the additional ME-induced field is very local, with a localization length defined by the MEN's average size of approximately $30 \mathrm{~nm}$. So, although the local induced fields are quite significant, the average effect in the relatively large NMR setup could be much smaller. According to this analysis, it can be seen that due to the ME effect, when an external magnetic field is swept in a 5-Oe range around the bias field during each NMR measurement, the measured spectrum should reflect contributions of MENs from different binding sites. That is the reason why we see a significant dependence on the cell type when MENs are placed into cancer media. Again, it can be noted that the traditional purely magnetic nanoparticles, which don't display any ME effect, couldn't provide this intrinsic contribution specific to each cell type, despite the fact that their saturation magnetization is almost two orders of magnitude higher than that for MENs. In a way, MENs allow to translate the intrinsic electric-field configuration on the cell membrane at the subcellular level into an easily measurable signature NMR signal specific to this particular cell type. 
The same physics could be used to explain the observed MENs-affected NMR spectra in the other two applications under study, i.e., for blood cell and bacteria identification. 


\section{CONCLUSION}

MENs could be used to distinguished between different types of bacteria, viruses, blood cells, cancer cells and their normal counterparts at the subcellular level.

The above experiments on bacteria, cancer cells, and prothrombin clearly showed that MENs could be used not only to distinguish between the organisms from their normal counterparts but also to identify different organisms. In other words, the experiment on cancer cells showed that MENs could be used not only to distinguish between cancer cells from their normal counterparts but also to identify different cancer cell lines. Different cells have different surface topography with different binding sites for nanoparticles. In addition, the surface morphology depends on the cell progression. In other words, the NMR signal modified by MENs in the cell media is complex and made of several components. The nanoparticles can be found on the cell membrane surface as well as inside the cell, as confirmed through AFM analysis. An important observation is the fact only MENs, due to the ME effect, could be used to provide such a signature identification at the subcellular level. For comparison, traditional magnetic nanoparticles with no ME effect couldn't provide such a precise diagnostic capability.

Optimization and proper calibration of MENs concentration, sample preparation, precise measurement and proper signal processing application design will further enhance the signature identification of biomolecules. It has been proven that exploitation of intrinsic surface electromagnetic properties of biological organism and utilization of the nuclear magnetic resonance property can enable a cost effective and portable tool for biomedical signature and screening. 


\section{FUTURE WORK}

Experimentally, we have shown the clustering of microorganisms with MENs and their magnetoelectric contribution leading to signature identification. We are working on the study of molecular interaction using free software called VMD (Visual Molecular Dynamics) and NAMD (Nanoscale Molecular Dynamics) simulation that can lead to an in-depth analysis of MENs interaction with microorganisms. Recently, theoretical biophysics group at FIU showed that Ebola virus protein hexamer enhances the clustering of certain lipids in the plasma membrane [77]. IBM group has shown the potential interference of protein-protein interactions by graphene using computation molecular dynamics [78]. Nobody has done any theoretical research on MENs interaction with protein. We are using PDB (Protein Data Bank) file of prothrombin to study the interaction of MENs that can not only potentially explain our experimental result but also lead to possible treatment of blood related diseases.

Moreover, we move forward from the use of magnetoelectric nanoparticles; paradigm shift from diagnostics to treatment. With the approved IACUC, we are currently working on in-vivo experiment of MENs for $\mathrm{PH}$ in mouse model. The arteries are complex in structure; however, we take a simple mechanical model and try to get the stiff PASMC back into normal.

The modal is based on the Navier-Stokes equation for incompressible flow, $\rho\left(\frac{\partial v}{\partial t}+v \cdot \nabla v\right)=-\nabla \mathrm{p}+\mu \nabla^{2} v+f$. Continuity equation for incompressible blood flow, $\nabla \cdot v=0$ ( $\rho$ being constant $)$ 
Finite Element Method (FEM) simulation modelling was performed to study the variation of blood pressure due to stiffness of arteries.

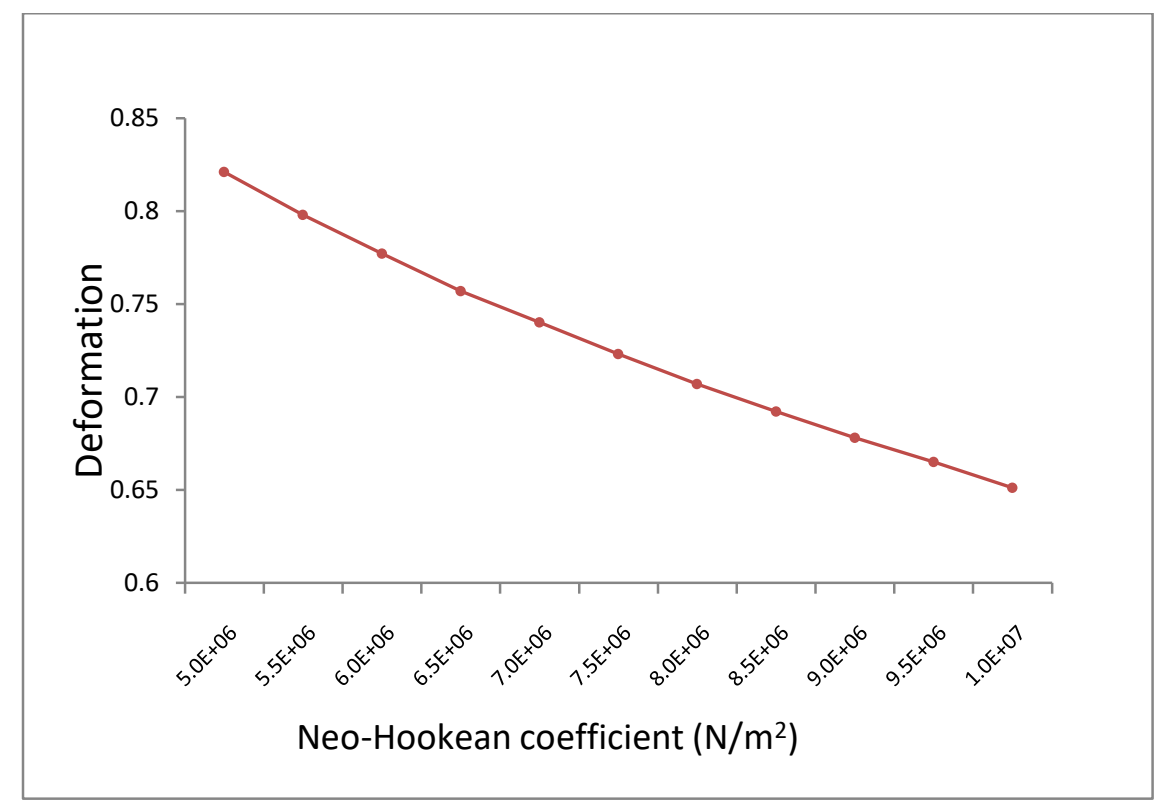

Figure 53: Deformation of arteries with neo-hookean coefficient.

The graph in Figure 53 shows that the stiff arteries get less relaxed due to the pressure of blood compared to normal arteries.

The magnetic dipole moment of PASMC depends on the magnetic orientation of the cell's magnetic dipole $d \sim \alpha^{\prime} H$ and $h \sim \frac{1}{d^{t}} \sim \frac{1}{\left(\alpha^{\prime} H\right)^{t}}$, (proposed Khanal's equation) where the value of $\mathrm{t}$ ranges from 0.3 to 2 .

Furthermore, most of the effective vasodilators, specially prostacyclin (PGI2) synthetic analogues have very low half life in plasma. Knowing the fact that less than $1 \%$ of current $\mathrm{PH}$ drugs is delivered to the targeted diseased PASMC. Our approach is to 
deliver $100 \%$ of the drug to specific cells. Firstly, we perform in vitro experiments to bind such PH drugs in the smart nanoparticles for targeted delivery on diseased cells. We approach to pre-program the self-triggering mechanism via defining the MENs saturation magnetization and the magneto-electric (ME) coefficient through a fabrication process or via application of an external magnetic field.

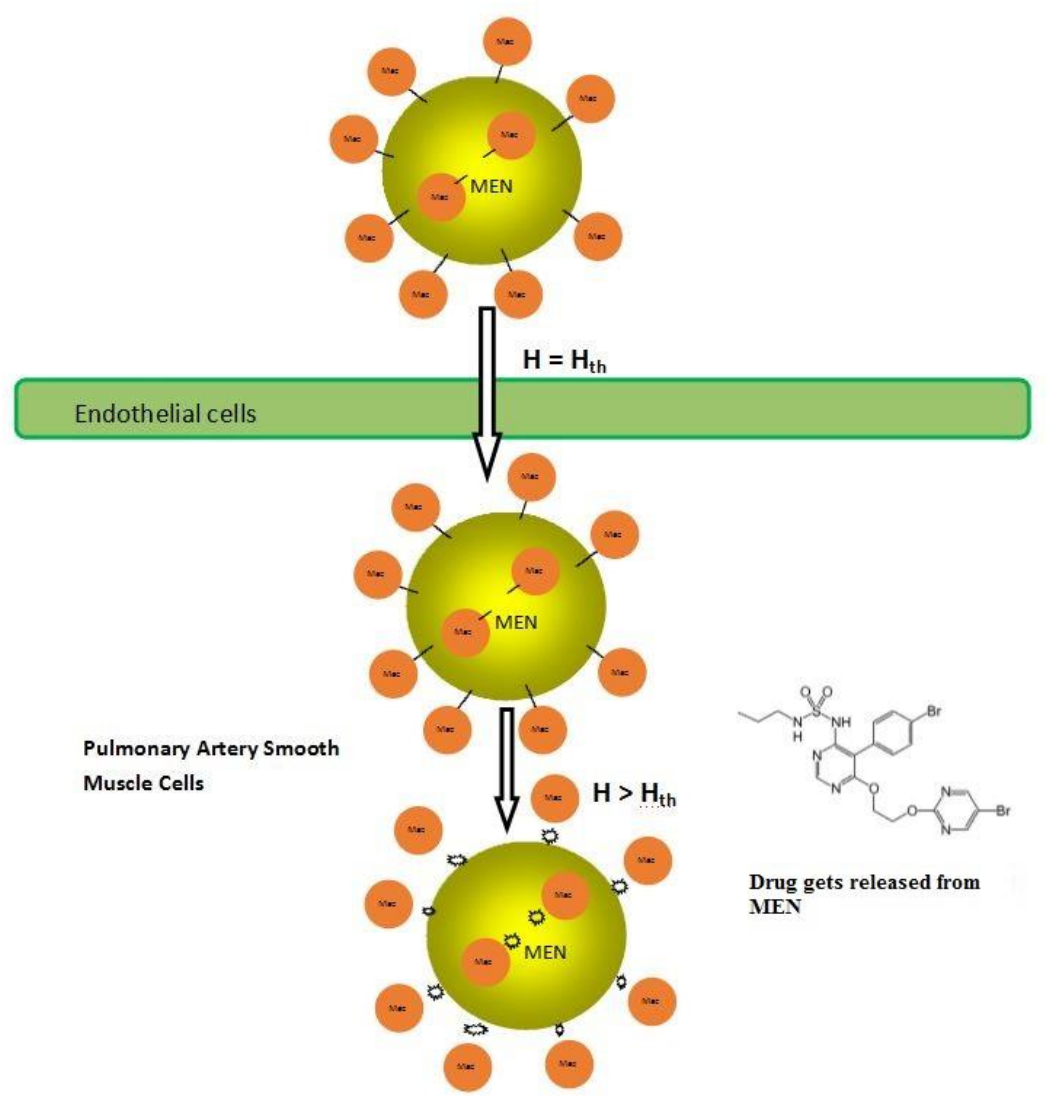

Figure 54: Schematic diagram of drug loaded MENs with critical field entering PASMC

Figure 54 depicts the MEN as field controlled nano-electroporation sites to let the drug pass through the endothelial cells and delivered to PASMC. At the threshold field $\mathrm{H}_{\mathrm{th}}$, 
the drug loaded MEN enters PASMC, and as the field increases further, the release of the drug from MEN is initiated.

In collaboration with theoretical biophysics research group at the department of physics at FIU and Miami Cardiovascular Institute, Baptist Health South Florida, we are in process of working on finding a new drug on MENs that can potentially cure PAH. 


\section{REFERENCES}

[1] Eerenstein W, Mathur ND, Scott JF. Multiferroic and magnetoelectric materials. Nature 2006;442:759-65.

[2] Guduru R, Liang P, Runowicz C, Nair M, Atluri V, Khizroev S. Magneto-electric nanoparticles to enable field-controlled high-specificity drug delivery to eradicate ovarian cancer cells. Sci Rep 2013;3:2953.

[3] Nair M, Guduru R, Liang P, Hong J, Sagar V, Khizroev S. Externally controlled ondemand release of anti-HIV drug using magneto-electric nanoparticles as carriers. Nat Commun 2013;4:1707.

[4] Rodzinski A, Guduru R, Liang P, Hadjikhani A, Stewart T, Stimphil E et al. Targeted and controlled anticancer drug delivery and release with magnetoelectric nanoparticles. Sci Rep 2016;6:20867.

[5] Bousquet E, Cano A. Non-collinear magnetism in multiferroic perovskites. J Phys Condens Matter 2016;28:123001.

[6] Liu XT, Chen WJ, Jiang GL, Wang B, Zheng Y. Diverse interface effects on ferroelectricity and magnetoelectric coupling in asymmetric multiferroic tunnel junctions: the role of the interfacial bonding structure. Phys Chem Chem Phys 2016;18:2850-8.

[7] Kaushik A, Tiwari S, Dev Jayant R, Marty A, Nair M. Towards detection and diagnosis of Ebola virus disease at point-of-care. Biosens Bioelectron 2016;75:254-72.

[8] Cai H, Parks JW, Wall TA, Stott MA, Stambaugh A, Alfson K et al. Optofluidic analysis system for amplification-free, direct detection of Ebola infection. Sci Rep 2015;5:14494.

[9] Bingham AM, Cone M, Mock V, Heberlein-Larson L, Stanek D, Blackmore C et al. Comparison of Test Results for Zika Virus RNA in Urine, Serum, and Saliva Specimens from Persons with Travel-Associated Zika Virus Disease - Florida, 2016. MMWR Morb Mortal Wkly Rep 2016;65:475-8.

[10] Roze B, Najioullah F, Ferge JL, Apetse K, Brouste Y, Cesaire R et al. Zika virus detection in urine from patients with Guillain-Barre syndrome on Martinique, January 2016. Euro Surveill 2016;21:10.2807/1560,7917.ES.2016.21.9.30154.

[11] Gourinat AC, O'Connor O, Calvez E, Goarant C, Dupont-Rouzeyrol M. Detection of Zika virus in urine. Emerg Infect Dis 2015;21:84-6. 
[12] Wang X, Zhou A, Cai W, Yu D, Zhu Z, Jiang C et al. Highly sensitive fluorescent stain for detecting lipopolysaccharides in sodium dodecyl sulfate polyacrylamide gel electrophoresis. Electrophoresis 2015;36:1795-800.

[13] Chen M, Cong WT, Zhou X, Zhu ZX, Ye WJ, Ling J et al. A method for sensitive staining of DNA in polyacrylamide gels using basic fuchsin. Bioanalysis 2013;5:154554.

[14] Strathmann M, Wingender J, Flemming HC. Application of fluorescently labelled lectins for the visualization and biochemical characterization of polysaccharides in biofilms of Pseudomonas aeruginosa. J Microbiol Methods 2002;50:237-48.

[15] Bassam BJ, Caetano-Anolles G, Gresshoff PM. Fast and sensitive silver staining of DNA in polyacrylamide gels. Anal Biochem 1991;196:80-3.

[16] Nugent RP, Krohn MA, Hillier SL. Reliability of diagnosing bacterial vaginosis is improved by a standardized method of gram stain interpretation. J Clin Microbiol 1991;29:297-301.

[17] Hitchcock PJ, Brown TM. Morphological heterogeneity among Salmonella lipopolysaccharide chemotypes in silver-stained polyacrylamide gels. J Bacteriol 1983;154:269-77.

[18] Tsai CM, Frasch CE. A sensitive silver stain for detecting lipopolysaccharides in polyacrylamide gels. Anal Biochem 1982;119:115-9.

[19] David N. Fredricks, David A. Relman. Application of Polymerase Chain Reaction to the Diagnosis of Infectious Diseases. Clinical Infectious Diseases 1999;29:475.

[20] Tsai YL, Olson BH. Rapid method for separation of bacterial DNA from humic substances in sediments for polymerase chain reaction. Appl Environ Microbiol 1992;58:2292-5.

[21] Yan Y, Warren SC, Fuller P, Grzybowski BA. Chemoelectronic circuits based on metal nanoparticles. Nat Nanotechnol 2016;11:603-8.

[22] Avouris P, Chen Z, Perebeinos V. Carbon-based electronics. Nat Nanotechnol 2007;2:605-15.

[23] Kosaka PM, Pini V, Ruz JJ, da Silva RA, Gonzalez MU, Ramos D et al. Detection of cancer biomarkers in serum using a hybrid mechanical and optoplasmonic nanosensor. Nat Nanotechnol 2014;9:1047-53.

[24] Duncan R, Gaspar R. Nanomedicine(s) under the microscope. Mol Pharm 2011;8:2101-41. 
[25] Gaster RS, Xu L, Han SJ, Wilson RJ, Hall DA, Osterfeld SJ et al. Quantification of protein interactions and solution transport using high-density GMR sensor arrays. Nat Nanotechnol 2011;6:314-20.

[26] Ilinskaya AN, Dobrovolskaia MA. Nanoparticles and the blood coagulation system. Part I: benefits of nanotechnology. Nanomedicine (Lond) 2013;8:773-84.

[27] Ilinskaya AN, Dobrovolskaia MA. Nanoparticles and the blood coagulation system. Part II: safety concerns. Nanomedicine (Lond) 2013;8:969-81.

[28] Hussain S, Vanoirbeek JA, Hoet PH. Interactions of nanomaterials with the immune system. Wiley Interdiscip Rev Nanomed Nanobiotechnol 2012;4:169-83.

[29] Di Gioacchino M, Petrarca C, Lazzarin F, Di Giampaolo L, Sabbioni E, Boscolo P et al. Immunotoxicity of nanoparticles. Int J Immunopathol Pharmacol 2011;24:65S$71 \mathrm{~S}$.

[30] Khabbaz RF, Moseley RR, Steiner RJ, Levitt AM, Bell BP. Challenges of infectious diseases in the USA. Lancet 2014;384:53-63.

[31] Alcantara D, Lopez S, Garcia-Martin ML, Pozo D. Iron oxide nanoparticles as magnetic relaxation switching (MRSw) sensors: Current applications in nanomedicine. Nanomedicine 2016;12:1253-62.

[32] Koh I, Josephson L. Magnetic nanoparticle sensors. Sensors (Basel) 2009;9:8130-45.

[33] Wang W, Ma P, Dong H, Krause HJ, Zhang Y, Willbold D et al. A magnetic nanoparticles relaxation sensor for protein-protein interaction detection at ultra-low magnetic field. Biosens Bioelectron 2016;80:661-5.

[34] Saritas EU, Goodwill PW, Croft LR, Konkle JJ, Lu K, Zheng B et al. Magnetic particle imaging (MPI) for NMR and MRI researchers. J Magn Reson 2013;229:1 16-26.

[35] Zhou Z, Leake MC. Force Spectroscopy in Studying Infection. Adv Exp Med Biol 2016;915:307-27.

[36] Grütter P, Mamin HJ, Rugar D. Magnetic Force Microscopy (MFM). In: Wiesendanger R, Guntherodt $\mathrm{H}$-, editors. Scanning Tunneling Microscopy II: Springer- Verlag Berlin Heidelberg; 1992, p. 151.

[37] Bae YH, Park K. Targeted drug delivery to tumors: myths, reality and possibility. J Control Release 2011;153:198-205. 
[38] Kamaly N, Xiao Z, Valencia PM, Radovic-Moreno AF, Farokhzad OC. Targeted polymeric therapeutic nanoparticles: design, development and clinical translation. Chem Soc Rev 2012;41:2971-3010.

[39] E. Ascher, H. Rieder, H. Schmid, and H. Stössel. Some Properties of Ferromagnetoelectric Nickel-Iodine Boracite, Ni3B7O13I. Journal of Applied Physics 1966;37:1404-5.

[40] Nan CW. Magnetoelectric effect in composites of piezoelectric and piezomagnetic phases. Phys Rev B Condens Matter 1994;50:6082-8.

[41] Guduru R. Bionano Electronics: Magneto-Electric Nanoparticles for Drug Delivery, Brain Stimulation and Imaging Applications. FIU Electronic Theses and Dissertations 2013.

[42] Boomgaard J, T. An in situ grown eutectic magnetoelectric composite material. J Mater Sci 1974;9:1705.

[43] Nan C-, Liu L, Cai N, Zhai J, Ye Y, Lin YH et al. A three-phase magnetoelectric composite of piezoelectric ceramics, rare-earth iron alloys, and polymer. Applied Physics Letters 2002;81:3831.

[44] Cai N, Nan C, Zhai J, Lin Y. Large high-frequency magnetoelectric response in laminated composites of piezoelectric ceramics, rare-earth iron alloys and polymer. Applied Physics Letters 2004;84:3516.

[45] Ryu J, Carazo AV, Uchino K, Kim H. <br />Magnetoelectric Properties in Piezoelectric and Magnetostrictive Laminate Composites. Japanese Journal of Applied Physics 2001;40:4948.

[46] Srinivasan G. Erratum: Magnetoelectric bilayer and multilayer structures of magnetostrictive and piezoelectric oxides [Phys. Rev. B , 214408 (2001)]. Physical review.B, Condensed matter 2002;66.

[47] Kida. Optical magnetoelectric effect of patterned oxide superlattices with ferromagnetic interfaces. Phys Rev Lett 2007;99:197404.

[48] Chen X. Magnetoelectric exchange bias systems in spintronics. Appl Phys Lett 2006;89:202508.

[49] Li P. A magnetoelectric energy harvester and management circuit for wireless sensor network. Sensors and actuators.A.Physical. 2010;157:100; 100,106; 106.

[50] Khitun A. Magnetoelectric spin wave amplifier for spin wave logic circuits. J Appl Phys 2009;106:123909. 
[51] BRACKE LPM. A broadband magneto-electric transducer using a composite material. International journal of electronics 1981;51:255; 255,262; 262.

[52] Scott JF. Data storage. Multiferroic memories. Nat Mater 2007;6:256-7.

[53] Roy A. Multiferroic Memories. J Appl Phys 2012;82:1; 1,12; 12.

[54] Petrov RV. Miniature antenna based on magnetoelectric composites. Electron Lett 2008;44:506; 506,508; 508.

[55] Sensenig R, Sapir Y, MacDonald C, Cohen S, Polyak B. Magnetic nanoparticle-based approaches to locally target therapy and enhance tissue regeneration in vivo. Nanomedicine (Lond) 2012;7:1425-42.

[56] Lee EA, Yim H, Heo J, Kim H, Jung G, Hwang NS. Application of magnetic nanoparticle for controlled tissue assembly and tissue engineering. Arch Pharm Res 2014;37:120-8.

[57] Torchilin VP. Drug Delivery Passive and Active Drug Targeting: Drug Delivery to Tumors as an Example. Drug Deliv 2010;197:3; 3,53; 53.

[58] Hirsjarvi S. Passive and Active Tumour Targeting with Nanocarriers. Current drug discovery technologies 2011;8:188; 188,196; 196.

[59] Alvarnas JC, Brown PA, Aoun P, Ballen KK, Barta SK, Borate U et al. Acute Lymphoblastic Leukemia, Version 2.2015. J Natl Compr Canc Netw 2015;13:124079.

[60] Locatelli F, Schrappe M, Bernardo ME, Rutella S. How I treat relapsed childhood acute lymphoblastic leukemia. Blood 2012;120:2807-16.

[61] Nguyen K, Devidas M, Cheng SC, La M, Raetz EA, Carroll WL et al. Factors influencing survival after relapse from acute lymphoblastic leukemia: a Children's Oncology Group study. Leukemia 2008;22:2142-50.

[62] Hayashi H, Hakuta Y. Hydrothermal Synthesis of Metal Oxide Nanoparticles in Supercritical Water. Materials 2010:3794.

[63] Williams DB, Carter CB. The Transmission Electron Microscope. 1996.

[64] Binnig G, Quate CF, Gerber C. Atomic force microscope. Phys Rev Lett 1986;56:9303.

[65] Cross SE, Jin YS, Rao J, Gimzewski JK. Nanomechanical analysis of cells from cancer patients. Nat Nanotechnol 2007;2:780-3. 
[66] Gascoyne PR, Wang XB, Huang Y, Becker FF. Dielectrophoretic Separation of Cancer Cells from Blood. IEEE Trans Ind Appl 1997;33:670-8.

[67] Lee H, Sun E, Ham D, Weissleder R. Chip-NMR biosensor for detection and molecular analysis of cells. Nat Med 2008;14:869-74.

[68] Benes J, Zatloukal J, Kletecka J. Viscoelastic Methods of Blood Clotting Assessment - A Multidisciplinary Review. Front Med (Lausanne) 2015;2:62.

[69] Poller L, Keown M, Ibrahim S, van den Besselaar AM, Roberts C, Stevenson K et al. Comparison of local International Sensitivity Index calibration and 'Direct INR' methods in correction of locally reported International Normalized Ratios: an international study. J Thromb Haemost 2007;5:1002-9.

[70] Levi M, Opal SM. Coagulation abnormalities in critically ill patients. Crit Care 2006;10:222.

[71] ERJ October Podcast: new guidelines for diagnosis and treatment of pulmonary hypertension. Eur Respir J 2015;46:E58.

[72] Garcia-Fernandez A, Marin F, Roldan V, Galcera-Jornet E, Martinez-Martinez JG, Valdes $\mathrm{M}$ et al. The HAS-BLED score predicts long-term major bleeding and death in anticoagulated non-valvular atrial fibrillation patients undergoing electrical cardioversion. Int J Cardiol 2016;217:42-8.

[73] Pozzi N, Chen Z, Di Cera E. How the Linker Connecting the Two Kringles Influences Activation and Conformational Plasticity of Prothrombin. J Biol Chem 2016;291:6071-82.

[74] Said K. Anticoagulation in pulmonary arterial hypertension: Contemporary data from COMPERA registry. Glob Cardiol Sci Pract 2014;2014:48-52.

[75] Olsson KM, Delcroix M, Ghofrani HA, Tiede H, Huscher D, Speich R et al. Response to letters regarding article, "Anticoagulation and survival in pulmonary arterial hypertension: results from the Comparative, Prospective Registry of Newly Initiated Therapies for Pulmonary Hypertension (COMPERA)". Circulation 2014;130:e110-2.

[76] Maron BA. Hemodynamics should be the primary approach to diagnosing, following, and managing pulmonary arterial hypertension. Can J Cardiol 2015;31:515-20.

[77] GC JB, Gerstman BS, Stahelin RV, Chapagain PP. The Ebola virus protein VP40 hexamer enhances the clustering of PI $(4,5) \mathrm{P} 2$ lipids in the plasma membrane. Physical chemistry chemical physics : PCCP 2016. 
[78] Luan B, Huynh T, Zhou R. Potential Interference of Protein-Protein Interactions by Graphyne. J Phys Chem B 2016;120:2124-31. 
VITA

\title{
CHOODA MANI KHANAL
}

\author{
Born, Ilam, Nepal
}

1998

B.Sc./ M.Sc., Physics

Tribhuvan University, Kathmandu, Nepal

2003-2005

M.S., Applied Physics

California State University Long Beach, CA

2007-2016

Doctoral Candidate, Electrical and Computer Engineering

Florida International University, Miami, FL

\section{PUBLICATIONS AND PRESENTATIONS}

1. Chooda Khanal, Magdalena Nawrocka, Xual Wang, Tao Liu, Roberto Panepucci (March 2009). Birefringence in Ring Resonator by Free Spectral Range and Wavelength measurement, American Physical Society March Meeting 2009, Pittsburg, PA.

2. C. Rodriguez, Chooda Khanal, Roberto R. Panepucci (May 2009). Nanoscale Hole Fabrication in Cylindrical Devices for Bubble Generation. 25 ${ }^{\text {th }}$ Southern Biomedical Engineering Conference 2009, IFMBE Proceedings, Vol. 24, pp 31-32.

3. Chooda Khanal, Garman Vargas, Kantesh Balani, Carmen Barbosa, Arvind Agarwal, Roberto Panepucci (March 2009). Metal Embedded Fiber Brag Grating Sensors. APS March Meeting 2009, Pittsburgh, PA.

4. Wang, L.B.; Selby, P.; Khanal, C.; Levin, G.; Haugan, T.J.; Barnes, P.N.; Kwon, C (June 2005). The distribution of transport current in YBCO coated conductor with zipper striations. IEEE Transactions on Applied Superconductivity, Vol. 15, Issue 2, $\operatorname{Pg} 2950-2953$.

5. P. Selby, C. Khanal, L. B. Wang, C. Kwon (March 2005). VTSLM reveals Current Distribution around Features in Striated YBCO. APS March Meeting 2005, Los Angeles, CA.

6. Choodamani Khanal and Simon George (August 2004). Physics Education in Nepal. APS Forum on Education, Sacramento, CA, AAPT Newsletter.

7. Chooda M Khanal (August 2005). Study of the Local Current Transport Properties of Striated Coated Conductor using Variable Temperature Scanning Laser Microscopy. Master's thesis, California State University, Long Beach, CA. 
8. J. Jimenez, M. Salinero, V. Pope, L. Reyes, C. Khanal, M. Matos, E. Stone (April 2016). Impact of Education Alone vs. Education and Medical Intervention vs. Standard Treatment in Re-Hospitalization for Heart Failure. The Journal of Heart and Lung Transplantation, Vol. 35, Issue 4, S272-S274.

9. Hima Zaman, Chooda Khanal, Danielle Roberts, Javier Jimenez (June 2016). Rescue therapy with oral prostacyclin (Orenitram) in an octogenarian female with refractory pulmonary arterial hypertension. Pulmonary Hypertension Association, PHA's International PH Conference and Scientific Sessions.

10. Chooda Khanal, Danielle Roberts, Elizabeth Stone, Hima Zaman, Javier Jimenez (June 2016). Who, Where and When: The 3W's World Experience in Pulmonary Hypertension Clinical Research. Pulmonary Hypertension Association, PHA's International PH Conference and Scientific Sessions.

11. Javier Jimenez, Chooda Khanal, Julie Cadet, Obinna Echeruo, Debra Knight, Marcos Fernandez, Alfredo J. Vazquez, Anne Bello, Jeannie Caudill, Harry Aldrich (November 2014). Tricuspid Tissue Annular Displacement (TTAD); a novel echocardiography parameter to evaluate right ventricular function. $33^{\text {rd }}$ Annual Echocardiography Symposium, BHSF, Miami, FL.

12. T. Stewart, R. Guduru, L. Salgueiro, E. Stimphil, P. Liang, A. Rodzinski, C. Khanal, A. Schally, and S. Khizroev. Magnetoelectric particles cross blood-brain barrier to deliver anti-tumor peptide to cancer cells with on-demand release. (manuscript in preparation).

13. C. Khanal, A. Rodzinski, T. Stewart, R. Guduru, and S. Khizroev. Physics Considerations in Using Magnetoelectric Nanoparticles for Medical Diagnostic and Rapid Screening. (manuscript in preparation).

14. C. Khanal, A. Nagesetti, A. Rodzinski, E. Stimphil, R. Guduru, P. Liang, I. Agoulnik, and S. Khizroev. Magnetoelectric Nanoparticles Influence Prothrombin Activity. (manuscript in preparation)

15. A. Nagesetti, A. Rodzinski, E. Stimphil, T. Stewart, C. Khanal, P. Wang, R. Guduru, P. Liang, I. Agoulnik, and S. Khizroev (2016). Multiferroic coreshell magnetoelectric nanoparticles as NMR sensitive nanoprobes for cancer cell detection. (submitted for publication). 